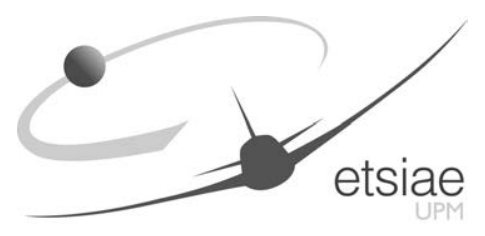

\title{
Correlation of spacecraft thermal mathematical models to reference data
}

Doctoral dissertation

Ignacio Torralbo Gimeno

\author{
Advisors: \\ Prof. Dr. Isabel Pérez Grande \\ Prof. Dr. Ángel Sanz Andrés
}



POLITÉCNICA

Tribunal nombrado por el Sr. Rector Magfco. de la Universidad Politécnica de Madrid, el día. de.. de $20 \ldots$...

Presidente:

Vocal:

Vocal:

Vocal:

Secretario:

Suplente:

Suplente:

Realizado el acto de defensa y lectura de la Tesis el día..........de........................de 20 ... en la E.T.S.I. /Facultad....

Calificación

EL PRESIDENTE

LOS VOCALES

EL SECRETARIO 



\section{Agradecimientos}

Quiero agradecer al IDR y en especial a Isabel por el esfuerzo dedicado en mi formación y darme la oportunidad de incorporarme al equipo de control térmico espacial. También a Ángel por sus ideas, que han sido la base de este trabajo.

No quiero pasar la oportunidad de agradecer a los compañeros de despacho que he tenido durante estos años. Con ellos he pasado los mejores momentos y sin duda todo el tiempo que hemos compartido se ve reflejado en esta tesis de una manera o de otra.

También quiero agradecer a mi familia y mi mujer MJ, por el apoyo que me he recibido durante todos estos años. 



\section{Abstract}

Model-to-test correlation is a frequent problem in spacecraft-thermal control design. The goal of correlation is to determine the values of the parameters of the thermal mathematical model (TMM) that allows reaching a good fit between the TMM results and test data. This way the uncertainty of the mathematical model is reduced. Quite often, this task is performed manually, mainly because a good engineering knowledge and experience is needed to reach a successful compromise, but the use of a mathematical tool could undoubtedly facilitate this work. The correlation process can therefore be considered as the selection of the model parameters that minimize the error of the model results with regard to the reference data.

In this doctoral dissertation, a simple method (GIPI) is presented. The method is suitable to solve the TMM-to-test correlation problem, using Jacobian matrix formulation and Moore-Penrose pseudo-inverse, generalized to include several load cases. Aside, in simple cases, this method also allows for analytical solutions to be obtained, which helps to analyze some problems that appear when the Jacobian matrix is singular.

To show the implementation of the method, two problems have been considered, one more academic, consisting of a simple 4-node model, and the other one a complex model, the TMM of the optical unit of PHI, one of the remote sensing instruments of the ESA mission Solar Orbiter. The method has given satisfactory reslts in both cases.

The convergence of the method has been studied and compared with several methods and the time required by this method (GIPI) subtantially outperforms the other methods. 



\section{Índice general}

1. Introducción 1

1.1. Control térmico y modelado térmico matemático en el ámbito espacial 2

1.1.1. Modelado térmico matemático . . . . . . . . . . . . . . . 4

1.1.2. Casos de análisis . . . . . . . . . . . . . . . 7

1.1.3. Modelos térmicos matemáticos reducidos . . . . . . . . . . . 8

1.1.4. Ensayos ambientales .............. . . 10

1.2. Correlación de modelos térmicos matemáticos . . . . . . . . . . . 11

1.3. Objetivo de la tesis . . . . . . . . . . . . . . . . 14

2. Formulación matemática $\quad 15$

2.1. Definición del error . . . . . . . . . . . . . . . . . . 15

2.2. Definición del problema . . . . . . . . . . . . . . 16

2.3. Análisis de un modelo de dos nodos . . . . . . . . . . . . . . . . 21

2.4. Actualización de modelos . . . . . . . . . . . . . 25

2.5. Generalización a varios casos de carga . . . . . . . . . . . . . 28

3. Ajuste de modelos térmicos matemáticos 31

3.1. Requisitos . . . . . . . . . . . . . . . . . 31

3.1.1. Características del modelo matemático . . . . . . . . . . . . . 32

3.1.2. Observabilidad de los parámetros . . . . . . . . . . . . . 32

3.1.3. Observabilidad de los resultados . . . . . . . . . . . . . 34

3.1.4. Elección e incertidumbre de las temperaturas de referencia . . 34

3.2. Algoritmo GIPI . . . . . . . . . . . . . . . . . . . 35 
3.2.1. Lectura de los datos . . . . . . . . . . . . . . . . 36

3.2.2. Selección de los parámetros y nodos del modelo . . . . . . . . 37

3.2.3. Ajuste de parámetros . . . . . . . . . . . . . . . . 38

4. Modelo 4 nodos 41

4.1. Caso indeterminado . . . . . . . . . . . . . . . . . . . . 43

4.2. Caso sobredeterminado . . . . . . . . . . . . . . . . . 47

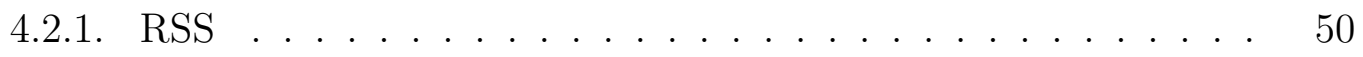

4.2.2. Actualización con 3 casos de carga . . . . . . . . . . 55

5. Aplicación a Solar Orbiter PHI O-Unit 59

5.1. Contexto . . . . . . . . . . . . . . . . . . . . . 59

5.2. Modelo térmico matemático . . . . . . . . . . . . . . . 61

5.2.1. Modelo térmico matemático detallado . . . . . . . . . . . 61

5.2.2. Modelo térmico matemático reducido . . . . . . . . . . 63

5.3. Ajuste del modelo . . . . . . . . . . . . . . . . . . . . . . . . . 69

5.3.1. Identificación de parámetros . . . . . . . . . . . . 73

5.3.2. Condiciones iniciales . . . . . . . . . . . . . . 76

5.3.3. Resultados . . . . . . . . . . . . . . . . . . . . 80

5.3.4. Influencia del coeficiente de variación máxima . . . . . . . 84

5.3.5. Comparación con otros métodos . . . . . . . . . . . . . 88

6. Conclusions and future work 93

6.1. Conclusions . . . . . . . . . . . . . . . . . . 93

6.2. Future Work . . . . . . . . . . . . . . . . . 96 


\section{Índice de figuras}

1-1. Esquema de las cargas térmicas externas de un satélite. . . . . . . . 5

1-2. Esquema de los elementos principales de un modelo térmico matemático. 7

1-3. DGMM y RGMM de la unidad electrónica de PHI [15] . . . . . . . . 9

2-1. Relación entre el espacio de parámetros y el de las temperaturas. . . . 20

2-2. Esquema de resistencias, modelo de 2 nodos . . . . . . . . . . . . . . 21

2-3. Esquema de resistencias de un modelo de 2 nodos simplificado. . . . . 24

3-1. Dependencia de la temperatura $T_{n}$ respecto al parámetro $X_{m}$. . . . 33

3-2. Diagrama del flujo del algoritmo de implementación del método. . . . 35

3-3. Diagrama de flujo del algoritmo de actualización parámetros. . . . . . 40

4-1. Esquema del modelo de 4 nodos empleado [36]. . . . . . . . . . . . . 42

4-2. Modelo de 4 nodos. Caso indeterminado. Evolución de la RSS. . . . . 45

4-3. Modelo de 4 nodos indeterminado. Evolución RSS. . . . . . . . . . . 47

4-4. Modelo de 4 nodos sobredeterminado. . . . . . . . . . . . . . . . . 48

4-5. Evolución del RSS en el modelo de 4 nodos. Caso sobredeterminado. . 49

4-6. RSS. Parámetros $X_{1}$ y $X_{2}$. . . . . . . . . . . . . . . . . . . . . . 51

4-7. RSS. Parámetros $X_{2}$ y $X_{5} \ldots \ldots \ldots \ldots \ldots$

4-8. RSS. Parámetros $X_{3}$ y $X_{5} \ldots \ldots \ldots \ldots \ldots$

4-9. RSS. Parámetros $X_{3}$ y $X_{6} \ldots \ldots \ldots \ldots \ldots$

4-10. RSS. Parámetros $X_{5}$ y $X_{6} \ldots \ldots \ldots \ldots$

4-11. RSS. Relación entre el RSS inicial y el número de iteraciones necesario para obtener un $\mathrm{RSS}<10^{-2}{ }^{\circ} \mathrm{C}$. . . . . . . . . . . . . . . 54 
4-12. Modelo de 4 nodos. Variación del RSS con el número de iteraciones .

4-13. Modelo de 4 nodos. Variación del valor de los parámetros de ajuste con el número de iteraciones . . . . . . . . . . . . . . . . 57

5-1. SO/PHI O-Unit con las mantas térmicas instaladas [62]. . . . . . . . 60

5-2. Modelo geométrico detallado. SO/PHI O-Unit. . . . . . . . . . 63

5-3. Modelo geométrico reducido. SO/PHI O-Unit. . . . . . . . . . . . . 64

5-4. PHI O-Unit RGMM (la MLI está retirada). . . . . . . . . . . . 65

5-5. Esquema de nodos y $G_{L}$ s. PHI O-Unit. . . . . . . . . . . 66

5-6. PHI OPT. Influencia parámetros. . . . . . . . . . . . . . . . . 74

5-7. PHI O-Unit. Influencia global $I_{X}$ de los parámetros de modelo. . . . . 75

5-8. PHI O-Unit. Influencia global de los parámetros. . . . . . . . . . . . . 76

5-9. Variación del RSS con el número de iteraciones. Influencia del coeficiente de variación máxima. . . . . . . . . . . . . . . . . . 86

6-1. SO/PHI O-Unit. Distribution . . . . . . . . . . . . . 95 


\section{Índice de tablas}

4.1. Modelo de 4 nodos. Temperaturas de referencia, $T_{R}$. . . . . . . . . 43

4.2. Modelo de 4 nodos. Parámetros de referencia, $X_{R}$. . . . . . . . . . 43

4.3. Modelo de 4 nodos. Parámetros iniciales en el caso indeterminado, $X_{0}$. 44

4.4. Modelo de 4 nodos. Evolución de temperaturas del modelo en el caso indeterminado. ..................... . . . . 44

4.5. Modelo de 4 nodos. Parámetros encontrados en el caso indeterminado. 45

4.6. Modelo de 4 nodos. Nuevos valores iniciales en el caso indeterminado, $X_{0} \ldots \ldots \ldots \ldots \ldots \ldots$

4.7. Modelo de 4 nodos. Evolución temperaturas del modelo en el caso indeterminado. ................... . . 46

4.8. Modelo de 4 nodos. Parámetros encontrados en el caso indeterminado. 46

4.9. Modelo de 4 nodos. Parámetros iniciales en el caso sobredeterminado. 48

4.10. Modelo de 4 nodos sobredeterminado. Temperaturas iniciales. . . . . 49

4.11. Modelo de 4 nodos. Evolución de las temperaturas obtenidas en con modelo en el caso sobredeterminado. . . . . . . . . . . . . . . . . . . 49

4.12. Modelo de 4 nodos. Evolución de los valores de los parámetros del modelo en el caso sobredeterminado. . . . . . . . . . . . . . . . . . 50

4.13. Valores iniciales de las diferentes trayectorias . . . . . . . . . . 50

4.14. Modelo de 4 nodos. Casos de carga. . . . . . . . . . . . . . . . 55

4.15. Modelo de 4 nodos. Temperaturas de referencia. . . . . . . . . . . 55

4.16. Modelo de 4 nodos. Temperaturas iniciales de los tres casos de carga. $\quad 56$

5.1. Identificación y número de nodos del modelo matemático de PHI O-Unit 62 
5.2. Nodos considerados en el RTMM. . . . . . . . . . . . . . . . . 68

5.3. Casos de carga. Cargas térmicas. . . . . . . . . . . . 70

5.4. Casos de carga. Temperatura de las interfaces . . . . . . . . . 70

5.5. Temperaturas de referencia. . . . . . . . . . . . . . . 72

5.6. PHI O-Unit. Valor inicial de los parámetros seleccionados. . . . . . 78

5.7. PHI O-Unit. Temperaturas iniciales. . . . . . . . . . . . . 79

5.8. PHI O-Unit. Valor final de los parámetros seleccionados. . . . . . . . 82

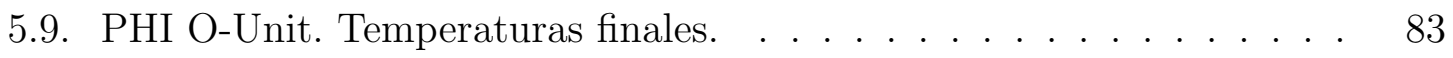

5.10. Flujos de calor a través de las interfaces en el caso DC2 (caliente operativo) . . . . . . . . . . . . . . . . . . 84

5.11. Flujos de calor a través de las interfaces en el caso PC1 (frío operativo). 84

5.12. RSS mínimo en 20 iteraciones . . . . . . . . . . . . . . . 87

5.13. Comparación de los diferentes métodos. . . . . . . . . . . . 90

5.14. PHI O-Unit. Comparación de los parámetros finales entre el método propuesto y el algoritmo genético. . . . . . . . . . . . . . . 91 


\section{Acrónimos}

AC Aperture Collar

APS Active Pixel Sensor

APSO Adaptive Particle Swarm Optimization

AR Acceptance Review

AU Astronomic Unit

BELA BepiColombo Laser Altimeter

BFGS Broyden, Fletcher, Goldfarb and Shanno algorithm

CC Contamination Collar

CDR Critical Design Review

CE Cold Element

CNES Centre National d'Études Spatiales

CP Connector Panel

CPC Common Power Converter

CT Correlation Tracker

DGMM Detailed Geometrical Mathematical Model

DTMM Detailed Thermal Mathematical Model

ECSS European Cooperation for Space Standardization

ELE Electronic Unit

ESA European Space Agency

FB Front Block

FDT Full Disk Telescope

FG Filtergraph

FPA Focal Plane Assembly

FS Field Stop

FSM Feed Select Mechanism

FT Feedthrough

GA Genetic Algorithm

GIPI Generalized Iterative Psudoinverse

GMM Geometrical Mathematical Model 


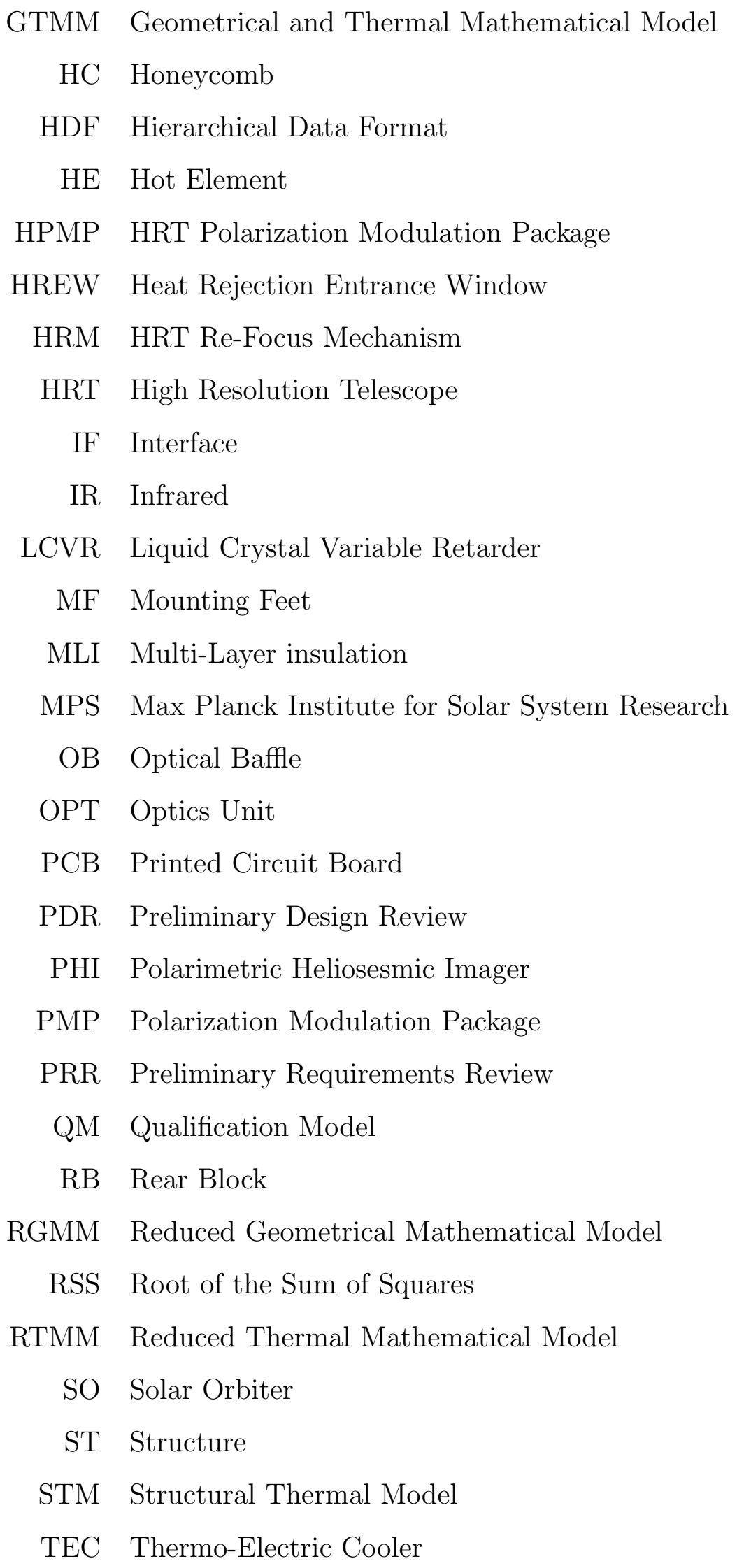


TMM Thermal Mathematical Model

TVAC Thermal Vacuum

UPM Universidad Politécnica de Madrid

URP Unit Reference Point 



\title{
Símbolos
}

\author{
A Área \\ C Vector de las capacidades térmicas \\ $c_{v}$ Coeficiente de variación máxima \\ $E_{T} \quad$ Error en las temperaturas \\ $G_{L} \quad$ Conductancia térmica lineal \\ $G_{R}$ Conductancia térmica radiativa \\ $i$ iterador \\ $\mathbf{I}_{\mathbf{X}}$ Matriz de influencia $\left[{ }^{\circ} \mathrm{C}\right]$ \\ j iterador \\ $k$ iterador \\ K Matriz de conductancias térmicas \\ M Matriz jacobiana
}

$\mathbf{M}_{\mathbf{c}}$ Matriz jacobiana cuadrada, $\mathbf{M}_{\mathbf{c}}=\mathbf{M}^{T} \mathbf{M}$

$\mathbf{M}^{+}$Pseudoinversa de Moore-Penrose

$N_{C} \quad$ Número de casos de carga

$N_{N} \quad$ Número de nodos

$N_{P} \quad$ Número de parámetros

$N_{T} \quad$ Número de temperaturas a ajustar

$\boldsymbol{Q}$ Vector de cargas térmicas

$Q_{i} \quad$ Carga térmica aplicada al nodo $i$

R Matriz de conductancias radiativas

$R_{i}^{L} \quad$ Conductacia radiativa linealizada

$\sigma$ Constante de Stefan-Boltman

$\boldsymbol{t}$ Vector de diferencia de temperaturas

$\boldsymbol{T}$ Vector de temperaturas

$\boldsymbol{T}^{\mathbf{0}} \quad$ Vector de temperaturas iniciales

$\boldsymbol{t}_{\boldsymbol{R}}$ Diferencia entre el las temperaturas de referencias y actuales

$\boldsymbol{T}_{\boldsymbol{R}} \quad$ Vector de temperaturas de referencia

$\boldsymbol{x}$ Vector diferencia entre el valor actual y el valor de referencia de los parámetros 
$\boldsymbol{x}^{*} \quad$ Vector de variaciones de los valores de los parámetros que generan las $\boldsymbol{T}_{\boldsymbol{R}}$

$x_{i} \quad$ Variación del parámetro $i$

$x_{i}^{\max } \quad$ Variación máxima permitida del parámetro $X_{i}$

X Parámetro

$\boldsymbol{X} \quad$ Vector de valores de los parámetros

$\boldsymbol{X}^{\mathbf{0}} \quad$ Vector de los valores iniciales de los parámetros

$X_{i}^{\max } \quad$ Valor máximo permitido del parámetro $X_{i}$

$X_{i}^{m i n} \quad$ Valor mínimo permitido del parámetro $X_{i}$

$X_{\text {final }}$ Valor final encontrado del parámetro

$X_{R_{i}} \quad$ Valor de referencia del parámetro $i$ 


\section{Capítulo 1}

\section{Introducción}

El control térmico espacial representa un rama muy importante en el diseño de instrumentos y vehículos espaciales. Su objetivo principal es asegurar que tanto el vehículo como todas las cargas de pago y componentes se encuentren dentro de su rango de temperatura especificado durante toda la misión $[1,2]$ ya sea operativo o no operativo según la fase en la que se encuentre. A diferencia de otras áreas de ingeniería, la dificultad e incluso imposibilidad en muchos casos, de probar los diseños en las mismas condiciones finales que van a experimentar, hacen que el diseño térmico, y por tanto, el modelado térmico matemático sea especialmente relevante.

Este modelado térmico matemático va evolucionando a lo largo de misión. A medida que van siendo disponibles más datos el modelo va evolucionando y conforme se van realizando ensayos se va validando experimentalmente. La información obtenida durante pruebas experimentales se utiliza de vuelta para alimentar el modelo matemático. Este proceso se conoce como correlación o actualización de modelos.

Más concretamente, correlacionar un modelo matemático consiste en ajustar los parámetros seleccionados del modelo, de manera que nos proporcione unos valores de las temperaturas calculadas lo más similar posible a los datos de referencia. En el caso de un ensayo de un instrumento o vehículo en cámara de vacío térmica, las temperaturas medidas serían los datos de referencia a emplear.

El proceso de correlacionar se hace tradicionalmente utilizando un método heurístico. Es el ingeniero responsable, que conoce bien el modelo matemático, el que en base 
a sus conocimientos previos va ajustando los parámetros del modelo. Este proceso tiende a ser largo y no siempre termina con los resultados óptimos.

Lo que se propone en esta tesis es desarrollar un método matemático que ayude a encontrar el mejor ajuste a realizar en los parámetros de manera que el modelo proporcione un resultado con el menor error posible en unas pocas iteraciones. Este método se aplicará para el ajuste de modelos térmicos matemáticos a datos de referencia. Estos datos de referencia pueden proceder de ensayos experimentales, otros modelos matemáticos e incluso información de vuelo.

\subsection{Control térmico y modelado térmico matemáti- co en el ámbito espacial}

Los proyectos espaciales van pasando por diferentes fases desde su concepción hasta el fin de la misión. Al final de cada una de estas fases es necesario pasar por una revisión para verificar que el diseño ha llegado a cierto grado de madurez. En el caso de trabajar dentro del marco de la Agencia Espacial Europea (ESA), estas fases están identificadas y estandarizadas [1-4]. El control térmico está presente en todas ellas con diferentes responsabilidades:

- Fase 0 - Análisis de misión. Se hace un análisis conceptual de la misión, se determinan qué elementos se van a necesitar principalmente y se plantea el dimensionamiento inicial. En esta fase los modelos matemáticos son únicamente pequeños cálculos para obtener las primeras cifras.

- Fase A - Viabilidad. Se analizan los requisitos térmicos generales de la misión. Se estudian los retos que se plantean y se comprueba la capacidad de solventarlos con las tecnologías actuales o en desarrollo. Esta fase culmina con el PRR (Preliminary Requirements Review).

- Fase B - Definición Preliminar. Durante está fase queda definido el alcance del control térmico de la misión así como el tipo de tecnología a usar (activa o pasiva). 
Se empiezan a desarrollar los primeros modelos matemáticos de los instrumentos y del vehículo e identifican los casos más relevantes de la misión a efectos térmicos.

Los modelos matemáticos están basados mayormente en cálculos y estimaciones habiendo unos pocos parámetros determinados. La mayor parte de los valores de estos parámetros se habrán obtenido mediante pruebas experimentales o heredados de misiones anteriores.

Esta fase termina con el PDR (Preliminary Design Review).

- Fase C - Definición detallada. El control térmico ya está completamente desarrollado y los modelos matemáticos cubren casi todos los escenarios posibles de la misión.

Durante esta fase se fabrica un modelo térmico estructural (STM) que es representativo en cuanto a potencia y propiedades físicas. Este modelo es sometido a diferentes pruebas (ensayos de vibración, térmicos en cámara de vacío, etc). Los datos obtenidos se utilizan para actualizar los modelos matemáticos tanto térmicos como estructurales.

Esta fase culmina con el CDR (Critical Design Review).

- Fase D - Producción y Calificación. Durante esta fase se fabrican los modelos de calificación para ser ensayados. Los modelos matemáticos empleados en el diseño tienen que ser actualizados y validados empleando los resultados de los ensayos y así poder utilizarlos tanto para predicción de nuevos ensayos como posterior ajuste. La información de estos modelos se pasa a los modelos generales para así poder re-evaluar las predicciones.

El final de esta fase se considera el paso por QR (Qualification Review) para el modelo de calificación y el AR (Acceptance Review) para el modelo de vuelo.

- Fase E - Utilización. Comienza la campaña de lanzamiento. Una vez comenzada la misión son los datos de vuelos los que se utilizan ahora como referencia para contrastar con el modelo matemático y ajustar los parámetros que sean necesarios.

- Fase F - Finalización. Se evalúan los datos obtenidos durante la misión para así 
perfeccionar los modelos en misiones futuras.

A lo largo de toda la vida de un proyecto espacial esta presente el ajuste o actualización de los modelos térmicos matemáticos $\left(\mathrm{TMM}^{1}\right)$ con datos medidos. Primero respecto a datos extraídos de ensayos de desarrollo, más tarde se utilizan datos provenientes de los ensayos tanto de modelos STM como QM y al final los datos utilizados son los de vuelo una vez que el vehículo ha sido lanzado.

El modelo térmico matemático va evolucionando a lo largo de las diferentes fases y lo hace también en complejidad. Esto supone un incremento considerable en potencia de cálculo necesaria y dificulta mucho la integración con modelos de otros subsistemas o equipos. Para poder facilitar esta tarea se recurre a modelos matemáticos reducidos $\left(\mathrm{RTMM}^{2}\right)$. Aquí es necesario de nuevo el ajuste, ya que estos RTMM se han de comportar y producir los mismos resultados que su modelo matemático detallado equivalente $\left(\mathrm{DTMM}^{3}\right)$. En este caso los datos de referencia que se utilizan no proceden de resultados experimentales sino de los modelos matemáticos detallados de los que derivan. Pero el proceso de ajuste es similar.

\subsubsection{Modelado térmico matemático}

En el ámbito espacial el método de modelado térmico más extendido es el método de lumped parameters $[1,2,5,6]$. Consiste en la discretización del sistema concentrándolo en un número finito de nodos. Cada nodo representa un volumen isotermo y tiene las propiedades acumuladas de éste, en particular la capacidad térmica. Los nodos están conectados entre ellos por medio de conductancias de manera análoga a las resistencias en circuito eléctrico. Aplicando la ecuación de balance de energía a cada nodo se obtiene:

$$
C_{i} \frac{d T_{i}}{d t}=Q_{i}+\sum_{j=1}^{n} G_{L i j}\left(T_{j}-T_{i}\right)+\sum_{j=1}^{n} G_{R i j} \sigma\left(T_{j}^{4}-T_{i}^{4}\right)
$$

\footnotetext{
${ }^{1}$ Thermal Mathematical Model.

${ }^{2}$ Reduced Thermal Mathematical Model.

${ }^{3}$ Detailed Thermal Mathematical Model.
} 
en donde se ha utilizado la notación del software ESATAN-TMS [7]. $C_{i}$ es la capacidad térmica, $G_{L i j}$ y $G_{R i j}$ son las conductancias lineales y radiativas entre los nodos $i$ y $j$ respectivamente. Las conductancias lineales representan los acoplamientos debidos a la conducción térmica y a la convección, si la hubiera, mientras que los términos de cuarto orden representan el intercambio de radiación térmica. $Q_{i}$ engloba el sumatorio de las cargas térmicas en el nodo $i$, tales como la carga solar, albedo planetario, radiación infrarroja terrestre o disipación eléctrica (ver Figura 1-1). De esta manera se genera un sistema de ecuaciones diferenciales ordinarias que se resolverá con métodos numéricos.

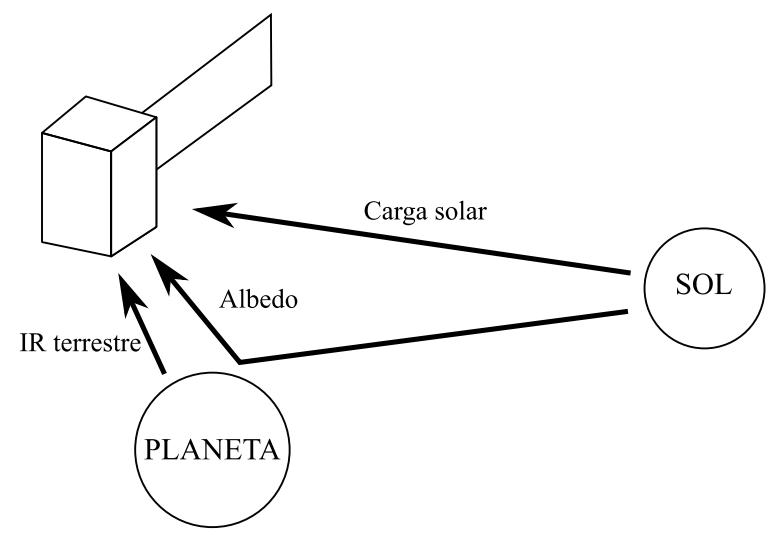

Figura 1-1: Esquema de las cargas térmicas externas de un satélite.

Los valores de $C_{i}, G_{L i j}$ y $G_{R i j}$ no siempre son conocidos, y deben evaluarse, ya que se derivan de los parámetros físicos tales como la geometría, densidad, conductividad térmica, par de apriete o acabado superficial.

Existen numerosos métodos para realizar el cálculo de conductancias $G_{L^{S}}$ automáticamente tales como Far Field, Extended Far Field o Mid-Section (muy bien explicados en [8-10]). Pero hay ocasiones en las que hay que hacer cálculos estimativos. Para ello se hace uso de simplificaciones e hipótesis que llevan asociada una cierta incertidumbre en el valor [11].

La conductancia lineal se calcula como una función del factor de forma y la conductividad térmica del material. Para una geometría rectangular (forma más simple) 
podemos expresar $G_{L}$ en la forma

$$
G_{L}=k \frac{A}{\Delta x}
$$

en donde $k$ es la conductividad térmica del material, $A$ es la sección transversal al flujo de calor y $\Delta x$ es la longitud. Vemos que un $G_{L}$ individual depende de al menos 3 parámetros, que son suceptibles de ser ajustados.

En el caso de los modelos matemáticos reducidos donde las geometrías se han simplificado drásticamente es más conveniente tratar los $G_{L} \mathrm{~S}$ como parámetros en sí (ver apartado 1.1.3) ya que han perdido una gran parte de su significado físico.

Cuando se desarrolla un modelo matemático, se desarrolla a la par un modelo geométrico matemático $\left(\mathrm{GMM}^{4}\right)$. De esta manera se facilita el cálculo de las cargas térmicas externas y de los factores de vista, que junto con el acabado superficial proporcionan los correspondientes $G_{R}$ s. Este cálculo se hace mediante el método de Monte Carlo [12] ya que en cuanto la geometría se complica es inviable hacerlo analíticamente. Hay que tener en cuenta que aunque una gran parte de las conductancias de los $G_{L}$ s y $G_{R}$ s son calculados directamente por el software, dependen de parámetros que en medida han sido estimados. Como ejemplo, considérese una unión atornillada entre dos partes, que puede estar representada por dos nodos, 1 y 2 (uno nodo por cada parte), su conductancia sería

$$
G L_{1,2}=f\left(k_{1}, k_{2}, h_{1,2}\right)
$$

El valor de la conductancia lineal, $G_{L 1,2}$, será una función de las conductividades térmicas $k_{1}$ y $k_{2}$, y de la conductancia de contacto en la unión, $h_{12}$. El valor de la conductividad térmica de los materiales es bien conocido, pero no así la conductancia de contacto. Mediante un ensayo se pueden medir las temperaturas de estos dos nodos y ajustar así el modelo matemático para que proporcionen estas temperaturas. Es decir, se determina el $G_{L 1,2}$ que proporciona unos resultados que se adaptan a la realidad. Para el ajuste se utiliza el parámetro $h_{1,2}$.

\footnotetext{
${ }^{4}$ Geometrical Mathematical Model
} 
Recapitulando, un modelo térmico matemático, TMM, se construye a partir de las propiedades físicas, disposición, acabados superficiales y montaje de los elementos. Con esta información se elabora el modelo geométrico, GMM, que junto con el modelado del entorno nos proporciona los acoplamientos radiativos, las cargas externas (IR planetario, albedo y solar) y las condiciones de contorno. Este flujo de información está esquematizado en la Figura 1-2. Todos estos datos que componen el modelo se pueden considerar parámetros y son susceptibles a ser ajustados más adelante.

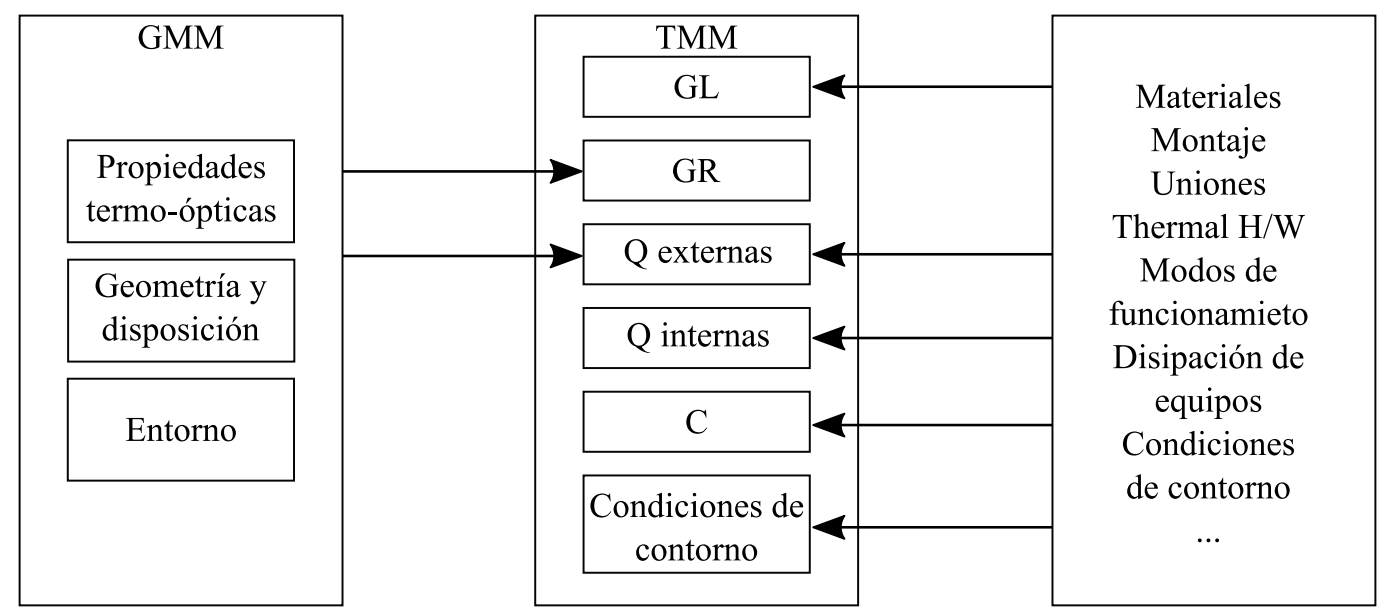

Figura 1-2: Esquema de los elementos principales de un modelo térmico matemático.

\subsubsection{Casos de análisis}

Durante las fases de diseño del control térmico en una misión espacial se identifican los casos de estudio más relevantes a efectos térmicos. Como regla general, un primer paso es evaluar las condiciones extremas de la misión. Se estudian así de manera aislada los extremos caliente y frío en estado estacionario. De esta manera aseguramos el éxito del control térmico en toda la misión, ya que las temperaturas siempre se encontrarán dentro de esta envolvente.

El estudio de casos estacionarios extremos es una práctica muy común [13]. La resolución del sistema de ecuaciones (1.1) queda simplificada al eliminar el término diferencial ya que el lado izquierdo de la ecuación pasa a ser cero al no haber variación con el tiempo. Las capacidades térmicas de los nodos, $C_{i}$ dejan de estar presentes en la ecuación y por lo tanto suponen un elemento menos de posibles errores. 
El método propuesto en esta tesis solo tiene en cuenta el ajuste de modelos térmicos matemáticos en casos en estado estacionario. Cada caso estacionario está determinado por unas condiciones de contorno específicas, representadas como $C_{k}$ con $k=1 \ldots N_{C}$, donde $N_{C}$ es el número de casos.

Si nos fijamos en la Figura 1-2 hay ciertos parámetros que se mantienen en los casos de análisis. Por ejemplo, si no hay partes móviles o variaciones en las propiedades termo-ópticas los $G_{R}$ s y los $G_{L}$ s se mantienen constantes. Es por ello que cada caso de análisis también se denomina caso de carga.

\subsubsection{Modelos térmicos matemáticos reducidos}

Una de las aplicaciones más relevantes del método que se presenta en esta tesis es el ajuste de los modelos térmico matemáticos reducidos (RTMM). Un RTMM es un modelo matemático derivado de otro más complejo, y que debe de producir idealmente los mismos resultados, en temperaturas y flujos de calor.

El proceso clásico de reducción, bien explicado en [14], consiste en condensar nodos y partes del modelo intentando preservar la topología y física del mismo. Esta reducción se hace tradicionalmente de forma manual por parte del ingeniero. El primer paso es reducir el modelo geométrico y a partir de él generar el RTMM. Después se procede a ajustar los parámetros de manera que los resultados sean lo más parecidos posible a los obtenidos con el DTMM. A priori no es posible determinar un conjunto de valores de los parámetros que proporcione resultados satisfactorios.

El RTMM al obviar muchos detalles hace que el cálculo de las conductancias térmicas entre nodos $\left(G_{L} \mathrm{~s}\right)$ sea una tarea muy complicada. Un caso bastante ilustrativo es la reducción del modelo matemático de la unidad electrónica mostrada en la Figura 1-3. En este caso se ha reducido de 4724 nodos a 7 nodos. Los detalles que se pierden en un proceso de reducción de este calibre son tan grandes que es muy difícil estimar el valor de $\operatorname{los} G_{L} \mathrm{~s}$.

El proceso de validación se hace comprobando que el RTMM da unos resultados lo más parecidos posible a los obtenidos mediante el DTMM y dentro de un margen de error determinado. Para ello se seleccionan los casos de análisis más relevantes en 

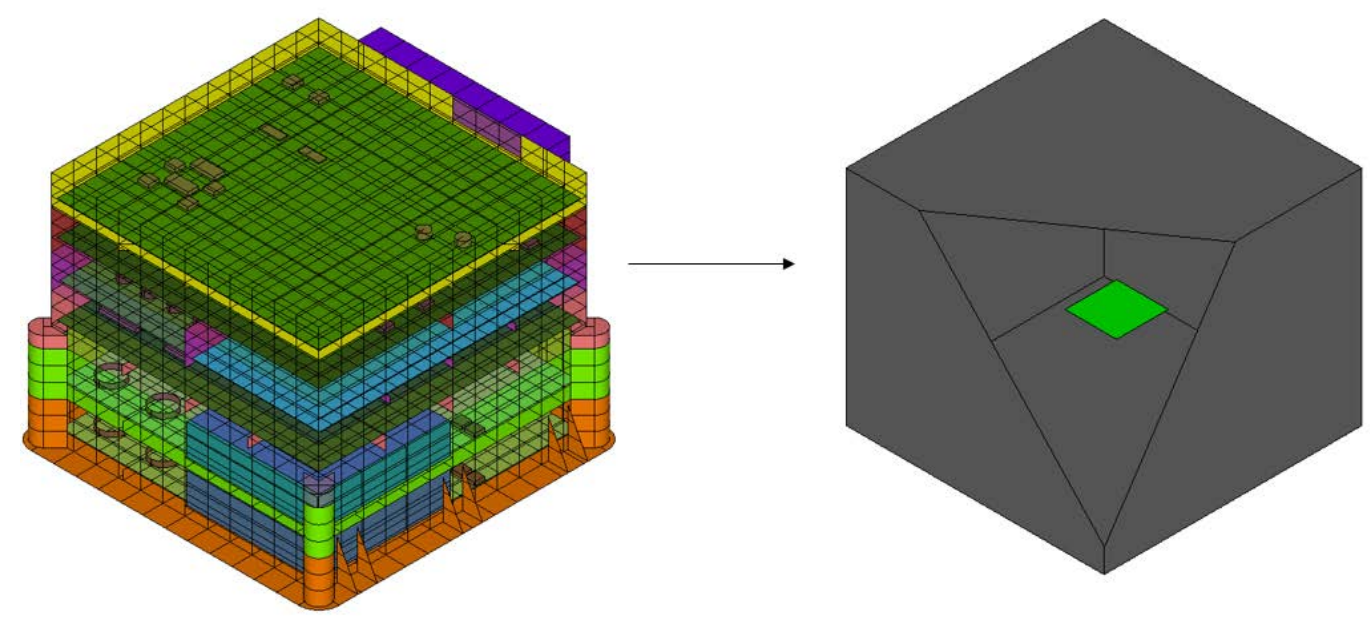

Figura 1-3: DGMM y RGMM de la unidad electrónica de PHI [15]

estado estacionario. Y en el caso que queramos verificar el valor de las capacidades térmicas de cada nodo también tendremos que comparar con un caso transitorio.

Todos los nodos presentes en el DTMM han de estar también representados en el RTMM. De esta manera, al ajustar el RTMM se utiliza la temperatura media ponderada con la capacidad térmica del grupo que representa ese nodo en el modelo reducido [4].

La generación de un modelo reducido implica dos partes diferenciadas:

1. Elaboración de un modelo con la capacidad de reproducir los resultados del modelo original.

2. Ajustar los parámetros del modelo de manera que reproduzca los resultados del modelo original.

Es muy importante tener en cuenta que si el RTMM no tiene la estructura correcta no va a ser posible ajustarlo de manera que reproduzca los resultados obtenidos por el DTMM.

Este proceso manual de reducción requiere de una gran cantidad de tiempo por parte del ingeniero, que puede durar desde una semana hasta más de un mes, dependiendo del modelo. Tanto la reducción como el ajuste se hace por el método de ensayo-error que no garantiza que se encuentre una solución optima. Es por ello, que ha habido muchos intentos de automatizarlo, [16-20] son ejemplos de ello. El problema que surge con estos métodos es que, si bien consiguen su objetivo, lo hacen a 
costa de perder el significado físico del modelo (por ejemplo conductancias térmicas negativas o conductancias que no existen en el original). Es por ello que en FernándezRico et al. [21] se desarrolla un método que se califica de casi automático. De esta manera mediante la intervención manual, asegura que el modelo reducido no pierda el sentido físico de los parámetros y que ciertas partes del modelo mantengan la representación geométrica que métodos completamente automáticos habrían condensado. En ocasiones es importante mantener la representación de ciertos subsistemas aunque térmicamente no afecten al modelo.

\subsubsection{Ensayos ambientales}

Los ensayos ambientales suponen una de las fuentes de obtención de datos de referencia más fiables a la hora de ajustar un modelo matemático con el comportamiento real. Los ensayos térmicos ambientales engloban los ensayos en cámara de vacío, los ensayos en cámara térmica presurizada con algún gas, frecuentemente nitrógeno, y los ensayos a presión ambiente. De éstos, los más interesantes a la hora de utilizar los datos para actualizar un modelo matemático son los ensayos en cámara de vacío térmica ya que no hay que tener en cuenta la convección. La cámara de vacío térmica se utiliza para realizar diferentes tipos de ensayos:

1. Ensayos de comportamiento. Se trata de someter los instrumentos a un entorno lo más parecido al que se van a encontrar durante la misión, de manera que se pueda verificar el funcionamiento.

2. Ciclados térmicos. Se trata de ciclar (calentar y enfriar) los equipos un número determinado de veces, dependiente del modelo, para verificar el correcto diseño $\mathrm{y}$ funcionamiento.

3. Ensayos de balance térmico. Se llevan los equipos hasta una temperatura determinada y un modo de funcionamiento y se espera hasta que se haya alcanzado el equilibrio térmico. Se pretende verificar y validar el modelo matemático y en ocasiones probar que los equipos son capaces de sobrevivir en las condiciones extremas para las que ha sido diseñado.

4. Ensayos de Bake-out y Outgassing. Se trata de eliminar las moléculas volátiles 
presentes en los instrumentos con el fin de limpiarlos y reducir así esta fuente de contaminación.

5. Ensayos de desarrollo. Estos ensayos se utilizan como apoyo al diseño de mecanismos y partes de los instrumentos. Son ensayos más rápidos y menos costosos con el fin de validar o tener medidas para el diseño.

De estos, los más relevantes para el ajuste son los ensayos de balance térmico. Al haber alcanzado el equilibrio térmico, se llega a un caso estacionario, que como se ha comentado en la sección 1.1.1 es lo que se va a utilizar para aplicar el método de actualización propuesto.

La planificación de los ensayos se convierte en un punto clave a la hora de usar los datos obtenidos para el ajuste del modelo térmico matemático. Es muy importante conocer el modelo y el efecto que tiene cada parámetro con el fin de posicionar de manera correcta los sensores de temperatura. Los datos obtenidos de un ensayo mal planificado pueden resultar inútiles a la hora de ajustar el modelo.

\subsection{Correlación de modelos térmicos matemáticos}

La correlación de modelos matemáticos es una tarea compleja no solo por el tiempo que requiere por parte del ingeniero, sino por la dificultad de encontrar un conjunto de valores de los parámetros que permitan al modelo reproducir los resultados correctos. El ajuste de los modelos manualmente $[22,23]$ sigue siendo una práctica común, si bien a lo largo de los últimos años ha habido numerosos intentos de automatizarla lo más posible [24-27]. Incluso ha habido esfuerzos por parte de la ESA junto con el Centre National D'Etudes Sapatiales (CNES) y Astrium R\&D [28-32] para explorar el uso de algoritmos genéticos (GA) para esta tarea. Las conclusiones de estos estudios apuntan que aunque los resultados son buenos, aún queda mucho trabajo hasta poder implementar en las herramientas usadas para cálculos térmicos un sistema autónomo completo en el ámbito del control térmico espacial.

El proceso de correlación puede considerarse un problema de optimización. Los 
problemas inversos ${ }^{5}$ globales de optimización no son nuevos en ciencia e ingeniería $[33,34]$, la dificultad no solo está en la puesta a punto de estos métodos, si no que la solución a la que se llega es dependiente del punto de comienzo, como describe Galski et al. [35]. Y en ocasiones se pierde el significado físico de los parámetros.

En el presente caso, hay algunas simplificaciones que se pueden tener en cuenta, que sugieren que el uso de métodos basados en el gradiente o la matriz jacobiana pueden ser los más acertados [36]. La propiedad más importante es que alrededor de un punto, el comportamiento de matemático suele ser suave y monótono con la variación de los parámetros. Es por ello que Klement [36] utiliza un método de Broyden basado en algoritmos cuasinewtonianos. La ventaja de su método respecto a otros es que es capaz de utilizar un vector de información en vez de un solo escalar en cada iteración. Este vector contiene las diferencias de temperatura entre el modelo y las mediciones. Aparte, Klement propone una variación sobre el 'Good Broyden's method' [37] y la actualización de los valores de la matriz jacobiana en cada iteración. Los resultados que obtiene muestran que después de alrededor de 6 iteraciones consigue un error menor a $10^{-3} \mathrm{~K}$.

Por otro lado están los métodos estocásticos. Anglada y Garmendia [26] han desarrollado un método basado en algoritmos genéticos (GA) como continuación de los estudios previos realizados para la ESA. Lo han puesto a prueba en un modelo de 7 nodos del instrumento TriboLAB con lo que han llegado a algunas conclusiones interesantes: por una parte, es posible tener ajustado el modelo con una buena correlación con varios conjuntos diferentes de parámetros, y por otra concluyen que si se utiliza solo un caso de análisis no se está seguro de que el modelo funcione bien para el resto de casos. Un punto interesante de su método es que les permite también ajustar el modelo en casos transitorios. Para obtener los resultados se requieren entre 800 y 2500 iteraciones. En [38] los mismos autores aplican el algoritmo genético a un modelo de TriboLAB pero esta vez de 47 nodos con un total de 117 parámetros. En este caso analizan diferentes casos de carga así como número de parámetros. Aunque los tiempos de ejecución no son cortos (del orden de horas), consiguen obtener resul-

\footnotetext{
${ }^{5}$ Los valores de algunos parámetros del modelo son obtenidos mediante datos observados.
} 
tados bastante satisfactorios con errores entorno a $\pm 0,5^{\circ} \mathrm{C}$. El problema que surge es que la solución obtenida no es única y en ocasiones se puede perder el significado físico de los parámetros a ajustar.

Una buena comparación de los resultados obtenidos usado los GA y tanto el algoritmo de Broyden como sus diferentes versiones propuestas por Klement está descrita en [24]. Para la comparación de resultados estos autores [26] utilizan tanto el modelo de TrioboLAB de 7 nodos como varias versiones del modelo de 4 nodos propuesto en [36]. A la vista de los resultados el algoritmo más rápido demuestra ser el modificado por Klement (K2014).

Otra aproximación interesante utilizando métodos estocásticos viene de la utilización de la optimización adaptativa por enjambre de partículas (APSO) [39]. Beck et al. en [40] aplican este método para correlacionar el modelo matemático del instrumento BELA (BepiColombo Laser Altimeter) con los resultados de la campaña de ensayos STM. Utilizan un modelo matemático con 650 nodos del que seleccionan 10 parámetros para el ajuste. Estos parámetros los tienen acotados, de manera que nunca se pueda perder el significado de la magnitud que representan. Utilizan entre 15 y 17 puntos de ajuste y 5 casos estacionarios de análisis. Los resultados, después de 50 horas y 600 iteraciones muestran una discrepancia de temperatura de $4.2 \pm 3.2{ }^{\circ} \mathrm{C}$.

En N. van Zijl et al. [41] se hace una comparación del ajuste obtenido de un modelo matemático de 129 nodos y 16 parámetros utilizando el método de Montecarlo, GA y APSO. Para ello utiliza 24 puntos de medida en los ensayos en cámara de vacío del instrumento, llegando a la conclusión de que el método más efectivo es el APSO debido a su velocidad de convergencia. Consigue un error medio de $1.1^{\circ} \mathrm{C}$. Destaca el hecho de que haciendo el ejercicio inverso, es decir, buscar unas temperaturas que ha generado el modelo con el conjunto de valores encontrado, ningún método es capaz de volver a encontrar dicho conjunto de nuevo. En decir, si se tiene un conjunto de temperaturas obtenidas con un conjunto de valores de parámetros conocidos, ningún método es capaz de encontrar dicho conjunto de parámetros conocidos aún utilizando dichas temperaturas. 


\subsection{Objetivo de la tesis}

A la vista de la revisión bibliográfica realizada y de la experiencia de trabajo en el ámbito del control térmico, donde se ha podido constatar la falta de un método automatizable que permita la correlación de los TMMs con resultados optimizados para diferentes casos de carga, el objetivo de la presente tesis es exponer el estado en el que se encuentra la correlación de modelos térmicos matemáticos con datos de referencia y presentar un método novedoso para llevar a cabo esta tarea. La intención es poder desarrollar una herramienta que se pueda integrar en el flujo de trabajo habitual del ingeniero de control térmico. El software comercial que se utiliza extensamente en Europa es ESATAN-TMS [7,42] y es por ello que en esta tesis se ha utilizado su nomenclatura y estructura de archivos.

En la correlación de modelos matemáticos en el ámbito espacial se utilizan varios casos de análisis diferentes, es por ello que resulta de suma importancia el poder añadir tantos como sea necesario, sin que esto suponga un incremento en el tiempo y la complejidad del cálculo. Se busca por lo tanto una formulación adecuada que permita utilizar varios casos de carga de manera sencilla. 


\section{Capítulo 2}

\section{Formulación matemática}

\subsection{Definición del error}

Por su uso posterior, y su utilidad, se ha considerado oportuno empezar por la definición del error existente entre los resultados obtenidos con un modelo matemático y un modelo de referencia. Siguiendo la línea propuesta en [36], se cuantifica la diferencia entre dos vectores como la norma euclidiana en un espacio vectorial de dimensión $N$, de manera que:

$$
N_{x_{12}}=\left|\boldsymbol{x}_{\mathbf{1}}-\boldsymbol{x}_{\mathbf{2}}\right|=\left[\sum_{j=1}^{N}\left(x_{1_{j}}-x_{2_{j}}\right)^{2}\right]^{1 / 2}
$$

Donde tanto $\boldsymbol{x}_{\mathbf{1}}$ como $\boldsymbol{x}_{\mathbf{2}}$ son vectores de dimensión $N$. Esta norma se va a designar como RSS, de su siglas en inglés square Root of the Sum of Squares. Se utilizará esta expresión para evaluar el error global o distancia de los resultados de un modelo matemático respecto a los datos de referencia. Como se ha indicado en el capítulo anterior, los datos de referencia pueden provenir de otro modelo (por ejemplo un modelo detallado) o pueden tratarse de datos medidos en ensayos.

Esta definición de error de un modelo térmico matemático respecto a unas temperaturas de referencia no nos dice nada respecto a si el ajuste se considera válido o no. La validez del ajuste está ligada a desviaciones máximas de la temperatura con- 
siderados en cada nodo en concreto. La validez del ajuste se considera en el apartado 5 , teniendo en cuenta que estos requisitos pueden variar según el proyeto.

Cuando se habla del error en esta tesis se hace referencia siempre a las desviaciones de los resultados del modelo respecto las temperaturas de referencia. No hay que confundir este error con la incertidumbre intrínseca del modelo matemático. Un modelo que ha sido ajustado respecto a unas temperaturas de referencia puede contribuir a diminuir la incertidumbre de sus resultados.

\subsection{Definición del problema}

El ajuste de modelos matemáticos se puede considerar un problema de optimización $[43,44]$. Se va a determinar una función objetivo cuyo valor se quiere minimizar. Esta función tiene que ser una representación matemática que contenga la dependencia de los parámetros cuyos valores se quieren ajustar y las temperaturas de referencia. En este caso se trata de encontrar la combinación de los valores de parámetros que minimicen el error entre los datos de referencia y los proporcionados por el modelo. Siguiendo la línea propuesta por otros autores [44,45] definimos la función objetivo a minimizar, como:

$$
E_{T}(\boldsymbol{X})=\sum_{j=1}^{N_{N}}\left(T_{R_{j}}-T_{j}(\boldsymbol{X})\right)^{2}
$$

donde $N_{N}$ es el número de puntos empleados para comparar las temperaturas, $T_{R_{j}}$ es la temperatura de referencia en el punto $j$ y $T_{j}(\boldsymbol{X})$ es la temperatura estimada obtenida mediante el modelo térmico matemático con el conjunto de parámetros $\boldsymbol{X}$. Con esta definición de la función objetivo (2.2) todos los nodos tienen el mismo peso en la evaluación del error.

Se quiere encontrar la dirección en la que la función (2.2) tiene la máxima pendiente descendiente, para ello se evalúa el gradiente $\nabla_{\boldsymbol{X}} E_{T}$, que vendrá dado por

$$
\frac{\partial E_{T}}{\partial X_{i}}=\sum_{j=1}^{N_{N}} \frac{\partial E_{T}}{\partial T_{j}} \frac{\partial T_{j}}{\partial X_{i}}=-2 \sum_{j=1}^{N_{N}}\left(T_{R j}-T_{j}(\boldsymbol{X})\right) \frac{\partial T_{j}(\boldsymbol{X})}{\partial X_{i}}, \quad i=1 \ldots N_{P}
$$


donde $N_{P}$ es la dimensión de $\boldsymbol{X}$ o el número de parámetros.

La sensibilidad que tiene el modelo a cada parámetro se puede determinar como el efecto que tiene cada parámetro $X_{i}$ sobre la temperatura estimada de cada punto. De esta manera se calcula el efecto de la variación de cada parámetro individual en las temperaturas del modelo. Escrito en forma matricial, cada columna representa la variación respecto a un parámetro y cada fila la temperatura de dichos nodos, y se denomina matriz de sensibilidad o matriz jacobiana,

$$
M_{j i}=\frac{\partial T_{j}(\boldsymbol{X})}{\partial X_{i}}
$$

con $N_{N}$ filas por $N_{P}$ columnas.

La condición de mínimo en la función objetivo (2.2) viene dada cuando se cumple que (2.3) es nulo, es decir

$$
\nabla_{\boldsymbol{X}} E_{T}(\boldsymbol{X})=0
$$

que forma un sistema de $N_{N}$ ecuaciones con $N_{P}$ parámetros desconocidos. A pesar de que este sistema de ecuaciones se puede escribir de forma explícita, no es lineal en la dependencia de los parámetros y encontrar la solución se convierte en un problema bastante complejo. A menudo, se usan métodos iterativos (como Steepest Descent o algoritmos tipo Broyden entre otros) para resolver el sistema de ecuaciones hasta llegar a una condición límite, consistente en que la norma del vector (2.5) sea inferior a un cierto valor preestablecido

$$
\left|\nabla_{\boldsymbol{X}} E_{T}\left(\boldsymbol{X}^{*}\right)\right|<\epsilon
$$

en donde $\epsilon<<1$ es el error máximo permitido y $\boldsymbol{X}^{*}$ es el conjunto de parámetros con el que se obtiene dicho error ${ }^{1}$.

En cuanto a los términos de la matriz $\mathbf{M}(2.4)$, se pueden calcular utilizando la

\footnotetext{
${ }^{1}$ Hemos definido $|\boldsymbol{x}|$ como la norma del vector $\boldsymbol{x}$.
} 
ecuación de resolución de un TMM linealizada y en estado estacionario.

$$
\mathrm{K} T=Q
$$

donde $\mathbf{K}$ es la matriz de conductancias térmicas, $\boldsymbol{T}$ es el vector de temperatura de los nodos y $\boldsymbol{Q}$ es el vector de cargas térmicas. Los elementos de $\mathbf{K}, K_{i j}=f(\boldsymbol{X})$ son dependientes de los parámetros de modelo.

Derivando (2.7) respecto a los parámetros $X_{i}$, se obtiene

$$
\left(\frac{\partial}{\partial X_{i}} \mathbf{K}\right) \boldsymbol{T}+\mathbf{K} \frac{\partial}{\partial X_{i}} \boldsymbol{T}=\frac{\partial}{\partial X_{i}} \boldsymbol{Q}
$$

Despejando el término $\frac{\partial \boldsymbol{T}}{\partial X_{i}}$ se obtiene la expresión para las sensibilidades

$$
\begin{aligned}
\frac{\partial}{\partial X_{i}} \boldsymbol{T}=\mathbf{K}^{-1}\left[\left(-\frac{\partial}{\partial X_{i}} \mathbf{K}\right) \boldsymbol{T}+\frac{\partial}{\partial X_{i}} \boldsymbol{Q}\right] & \\
& =\mathbf{K}^{-1}\left[-\left(\frac{\partial}{\partial X_{i}} \mathbf{K}\right) \mathbf{K}^{-1}+\frac{\partial}{\partial X_{i}}\right] \boldsymbol{Q}
\end{aligned}
$$

donde se ha empleado (2.7) para sustituir $\boldsymbol{T}$. De manera que (2.9) se puede expresar como

$$
\frac{\partial}{\partial X_{i}} \boldsymbol{T}=\mathbf{S}_{\mathbf{i}} \boldsymbol{Q}
$$

que se corresponde con el vector columna del la matriz jacobiana $\mathbf{M}$, donde se define el operador $\boldsymbol{S}_{\boldsymbol{i}}$

$$
\boldsymbol{S}_{\boldsymbol{i}}=\mathbf{K}^{-1}\left(-\left(\frac{\partial}{\partial X_{i}} \mathbf{K}\right) \mathbf{K}^{-1}+\frac{\partial}{\partial X_{i}}\right)
$$

que depende únicamente de las conductacias del modelo. Este resultado es muy interesante ya que se observa una dependencia lineal de los vectores de las sensibilidades (columnas del jacobiano) con la carga $\boldsymbol{Q}$. Este resultado se puede utilizar para acelerar los cálculos en los problemas en los que el número de casos de carga sea elevado. 
De esta manera se puede determinar la sensibilidad de cada caso por separado y combinarlos posteriormente.

En el proceso de actualización de un modelo térmico matemático se pueden definir dos tipos de errores. El primero es el que ya se ha identificado, y se encuentra en el espacio de las temperaturas, viene determinado por la ecuación (2.2). El otro error se identifica en el espacio de los parámetros (o espacio de búsqueda). Al igual que $E_{T}$, se define como:

$$
E_{\boldsymbol{X}}=\sum_{i}^{N_{P}}\left(X_{R_{i}}-X_{i}\right)^{2}
$$

donde $X_{R_{i}}$ son los valores de los parámetros que nos estiman las temperaturas de referencia.

En el caso de los modelos térmicos matemáticos en un entorno localizado, en primera aproximación se puede suponer que existe una dependencia lineal y monótona entre las coordenadas en el espacio de las soluciones $\boldsymbol{T}$ y en el espacio de los parámetros $\boldsymbol{X}$. De manera que se puede definir una tranformación lineal entre ambos conjuntos. Aproximando las temperaturas en el entorno de una solución inicial $T^{0}$ (expansión en serie de Taylor, [46]) se puede escribir:

$$
T_{i}=T_{i}^{0}+\sum_{j=1}^{N_{P}}\left(\left(X_{j}-X_{j}^{0}\right) \frac{\partial T_{i}}{\partial X_{j}}\right)+\ldots
$$

Para simplificar el planteamiento (2.13) se puede expresar en función de las fluctuaciones entorno al punto inicial $\boldsymbol{T}^{0}=\boldsymbol{T}^{\mathbf{0}}\left(\boldsymbol{X}^{0}\right)$ haciendo,

$$
t_{i}=T_{i}-T_{i}^{0} \quad x_{j}=X_{j}-X_{j}^{0}
$$

determinándose

$$
t_{i}=\sum_{j=1}^{N_{P}} \frac{\partial t_{i}}{\partial x_{j}} x_{j} \quad i=1 \ldots N_{N}
$$


donde $\boldsymbol{t}$ y $\boldsymbol{x}$ son las fluctuaciones en el espacio de las temperaturas y en el espacio de los parámetros, respectivamente. La ecuación (2.15) se puede escribir en forma matricial,

$$
\boldsymbol{t}=\mathrm{M} \boldsymbol{x}
$$

donde $\mathbf{M}$ es la matriz jacobiana, definida previamente en (2.4):

$$
\mathbf{M}=\left[\begin{array}{cccc}
\frac{\partial t_{1}}{\partial x_{1}} & \frac{\partial t_{1}}{\partial x_{2}} & \cdots & \frac{\partial t_{1}}{\partial x_{N_{P}}} \\
\cdots & \ldots & & \cdots \\
\frac{\partial t_{N_{N}}}{\partial x_{1}} & \frac{\partial t_{N_{N}}}{\partial x_{2}} & \cdots & \frac{\partial t_{N_{N}}}{\partial x_{N_{P}}}
\end{array}\right]
$$

En la Figura 2-1 se muestra un esquema de esta transformación entre el espacio de
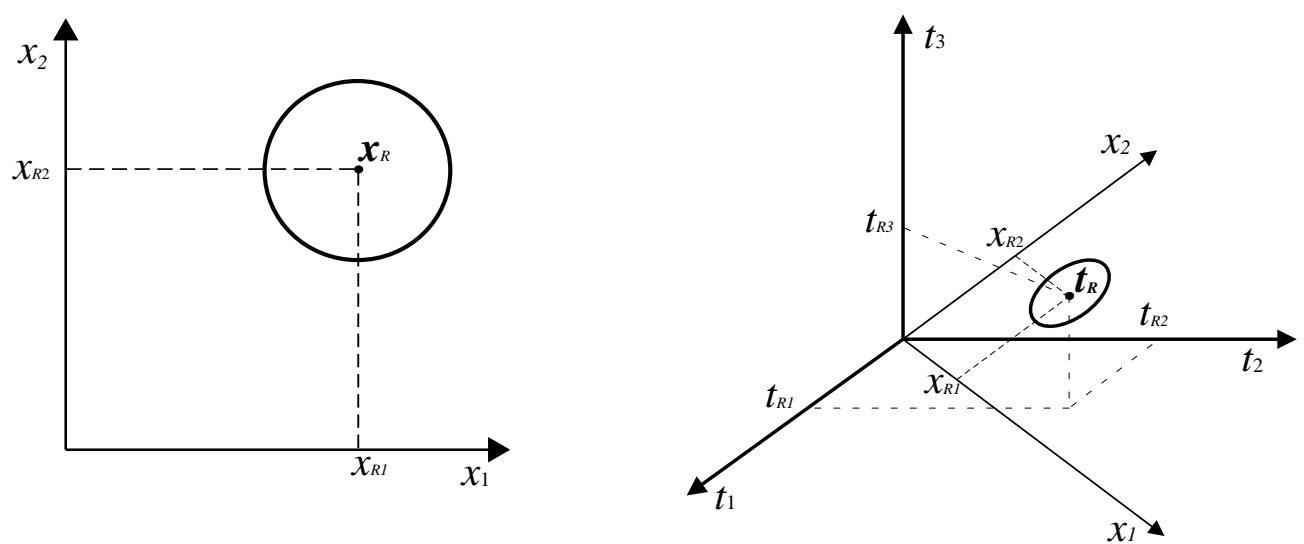

Figura 2-1: Relación entre el espacio de parámetros y el de las temperaturas.

búsqueda y el espacio de las temperaturas definido en (2.16). En esta Figura y con el fin de ayudar a la visualización se ha limitado el espacio de búsqueda a dos dimensiones (correspondiente a dos parámetros, $X_{1}$ y $X_{2}$ ) y el espacio de las temperaturas a tres dimensiones.

Como se ha deducido de (2.10) la matriz $\mathbf{M}_{N_{N} \times N_{P}}$ es constante para cada caso de carga. Analizando detalladamente su estructura, se puede distinguir entre diferentes casos: 
1. Si M es cuadrada y no singular, (2.16) representa una transformación biunívoca entre $\boldsymbol{t}$ y $\boldsymbol{x}$. El problema de minimización en el espacio de búsqueda (2.12) y de los resultados (2.2) es el mismo.

2. Si la dimensión de $\boldsymbol{x}$ es menor que $\boldsymbol{t}$, no se puede acceder a todos los puntos de $\boldsymbol{t}$ variando $\boldsymbol{x}$. La imagen de $\boldsymbol{x}$ no cubre todo $\boldsymbol{t}$ sino que forma un subespacio. Si el modelo matemático incluye los parámetros adecuados, se podrá estar dentro del subespacio de $\boldsymbol{t}$ que contiene la solución y por lo tanto se podrá llegar a tener un error nulo.

3. En el caso que la imagen de $\boldsymbol{x}$ en $\boldsymbol{t}$ no contenga la solución $\boldsymbol{T}_{R}$, nunca se va a poder obtener un error nulo. Esto se puede deber a:

a) El modelo matemático no tiene la arquitectura correcta, y no es posible obtener $\boldsymbol{T}_{R}$ con los parámetros $\boldsymbol{X}$ existentes.

b) No se han seleccionado bien los parámetros del espacio de búsqueda $\boldsymbol{X}$, a pesar que el modelo matemático esté bien planteado.

\subsection{Análisis de un modelo de dos nodos}

Con el fin de ayudar a explicar lo desarrollado en la sección anterior se va a usar un modelo térmico matemático simple. En la Figura 2-2 se muestra un esquema gráfico del modelo que se va a analizar. Consta de dos nodos y un parámetro de ajuste $K_{12}$. $Q_{1}$ y $Q_{2}$ representan las cargas internas disipadas en cada nodo y $R_{1}$ y $R_{2}$ las conductancias radiativas de cada nodo con el entorno. Lo primero que se

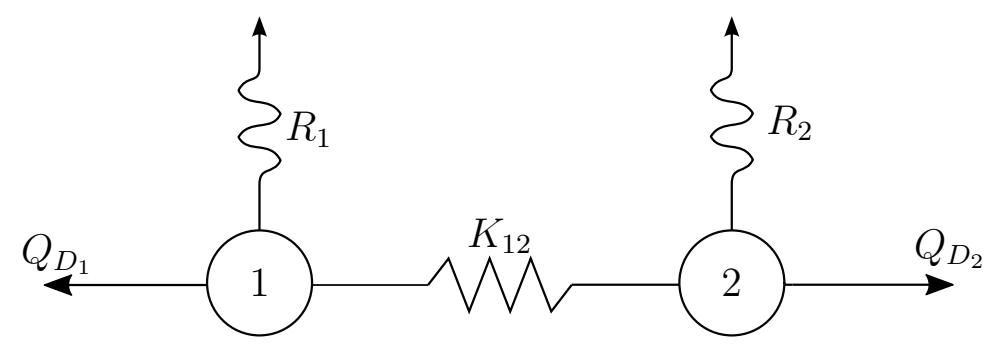

Figura 2-2: Esquema de resistencias, modelo de 2 nodos

hace es plantear la ecuación de balance energético en cada nodo. Para ello se linealiza la expresión del término de radiación entorno a una temperatura $\boldsymbol{T}^{\mathbf{0}}$, cercana a la 
solución que se busca. El intercambio de calor por radiación linealizado [47, 48] se expresa como $R_{i}^{L}$, y la disipación interna y la intercambiada por radiación está escrita como $Q_{i}=Q_{D_{i}}+Q_{R_{i}}$. De esta forma, las ecuaciones del balance energético quedan:

$$
\begin{aligned}
& K_{12}\left(T_{2}-T_{1}\right)-R_{1}^{L} T_{1}=Q_{1} \\
& K_{12}\left(T_{1}-T_{2}\right)-R_{2}^{L} T_{2}=Q_{2}
\end{aligned}
$$

que se pueden escribir en forma matricial, como $\mathbf{K T}=Q$ :

$$
\left[\begin{array}{cc}
-K_{12}-R_{1}^{L} & K_{12} \\
K_{12} & -K_{12}-R_{2}^{L}
\end{array}\right]\left[\begin{array}{l}
T_{1} \\
T_{2}
\end{array}\right]=\left[\begin{array}{c}
Q_{1}^{*} \\
Q_{2}^{*}
\end{array}\right]
$$

Teniendo en cuenta que en este caso solo se dispone de un parámetro de ajuste, $K_{12}$ el error (2.2) a minimizar queda en la forma

$$
E_{T}\left(K_{12}\right)=\left(T_{R_{1}}-T_{1}\left(K_{12}\right)\right)^{2}+\left(T_{R_{2}}-T_{2}\left(K_{12}\right)\right)^{2}
$$

de manera que la condición $(2.5), \nabla_{K_{12}} E_{T}=\mathbf{0}$ resulta ser

$$
\begin{aligned}
& \frac{\partial T_{T}}{\partial K_{12}}=-\left(T_{R_{1}}-T_{1}\right) \frac{\partial T_{1}}{\partial K_{12}}-\left(T_{R_{2}}-T_{2}\right) \frac{\partial T_{2}}{\partial K_{12}}=0 \\
& {\left[\boldsymbol{T}_{\boldsymbol{R}}-\boldsymbol{T}\right]^{T} \frac{\partial}{\partial K_{12}} \boldsymbol{T}=0}
\end{aligned}
$$

Para determinar los términos $\partial T_{i} / \partial K_{12}$, que se corresponden con los elementos del jacobiano, se usa la expresión (2.10) y se tiene en cuenta que $\boldsymbol{Q}$ no depende de $K_{12}$, 
de manera que:

$$
\begin{aligned}
\frac{\partial \boldsymbol{T}}{\partial K_{12}} & =-\mathbf{K}^{-1}\left[-\frac{\partial \mathbf{K}}{\partial K_{12}}\right] \mathbf{K}^{-1} \boldsymbol{Q} \\
& =-\frac{1}{\operatorname{det}(\mathbf{K})} \operatorname{adj}(\mathbf{K})\left[-\frac{\partial \mathbf{K}}{\partial K_{12}}\right] \mathbf{K}^{-1} \boldsymbol{Q} \\
& =-\frac{1}{\operatorname{det}(\mathbf{K})^{2}} \operatorname{adj}(\mathbf{K})\left[-\frac{\partial \mathbf{K}}{\partial K_{12}}\right] \operatorname{adj}(\mathbf{K}) \boldsymbol{Q} \\
& =-\frac{1}{\operatorname{det}(\mathbf{K})^{2}}\left[\begin{array}{ll}
-Q_{1} R_{2}^{2} & Q_{2} R_{1} R_{2} \\
-Q_{2} R_{1}^{2} & Q_{1} R_{1} R_{2}
\end{array}\right]
\end{aligned}
$$

La temperatura $\boldsymbol{T}$, se obtiene de (2.7):

$$
\begin{aligned}
\boldsymbol{T} & =\mathbf{K}^{-1} \boldsymbol{Q} \\
& =\frac{1}{\operatorname{det}(\mathbf{K})} \operatorname{adj}(\mathbf{K}) \boldsymbol{Q} \\
& =\frac{1}{\operatorname{det}(\mathbf{K})}\left[\begin{array}{c}
-Q_{1}\left(K_{12}+R_{2}\right)-Q_{2} K_{12} \\
-Q_{1} K_{12}-Q_{2}\left(K_{12}-R_{1}\right)
\end{array}\right]
\end{aligned}
$$

Utilizando (2.23) y (2.24) en (2.22), se llega a:

$$
\begin{aligned}
& \left(T_{R_{1}}+\frac{1}{\operatorname{det}(\mathbf{K})}\left(Q_{1}\left(K_{12}+R_{2}\right)+Q_{2} K_{12}\right)\right)\left(-Q_{1} R_{2}+Q_{2} R_{1}\right) R_{2}+ \\
& \quad+\left(T_{R_{2}}+\frac{1}{\operatorname{det}(\mathbf{K})}\left(Q_{2}\left(K_{12}-R_{1}\right)+Q_{1} K_{12}\right)\right)\left(Q_{2} R_{1}-Q_{1} R_{2}\right) R_{1}=0
\end{aligned}
$$

La resolución de esta ecuación determina el valor del parámetro $K_{12}$ que ajusta el modelo. Analizando el jacobiano (2.23) se pueden sacar interesantes comportamientos del modelo:

- Si ambas sensibilidades son cero $\left(\partial T_{1} / \partial K_{12}=\partial T_{2} / \partial K_{12}=0\right)$, significa que no se puede utilizar $K_{12}$ como parámetro para ajustar nuestro modelo.

- Si una de ellas es cero, no se podrá ajustar la temperatura correspondiente.

Para concretar más, se va a estudiar ahora un caso particular. Se considera que $R_{2}=0$ y $Q_{1}=0$ como se muestra en la Figura 2-3. 


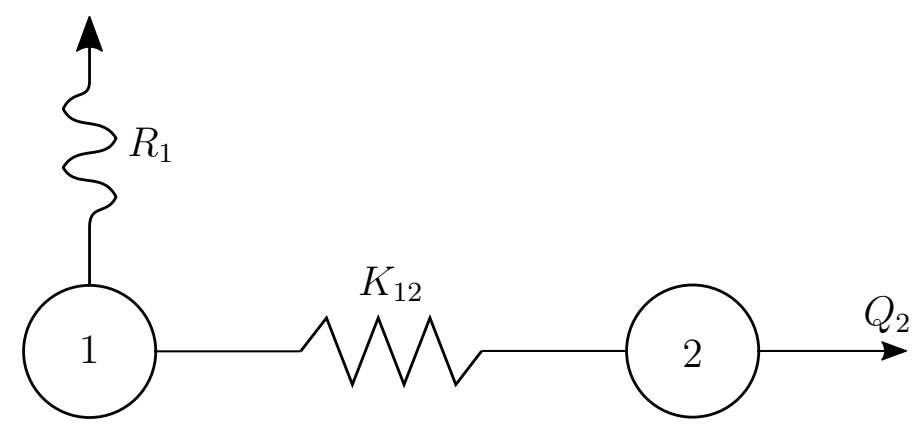

Figura 2-3: Esquema de resistencias de un modelo de 2 nodos simplificado.

De esta manera (2.22) se simplifica a:

$$
T_{R_{2}}+\frac{1}{\operatorname{det}(\mathbf{K})}\left(K_{12}+R_{1}\right) Q_{2}=0
$$

de donde se puede despejar $K_{12}$,

$$
\begin{aligned}
\frac{T_{R_{2}}}{Q_{2}} & =-\frac{\left(K_{12}+R_{1}\right)}{K_{12} R_{1}}=-\frac{1}{K_{12}}-\frac{1}{R_{1}} \\
\frac{1}{K_{12}} & =-\frac{T_{R_{2}}}{Q_{2}}-\frac{1}{R_{1}} \\
K_{12} & =\frac{1}{-\frac{T_{R_{2}}}{Q_{2}}-\frac{1}{R_{1}}}
\end{aligned}
$$

utilizando este valor de (2.27) se calculan las temperaturas del $T_{1}$ y $T_{2}$ a partir de $(2.24)$ :

$$
\begin{aligned}
& T_{1}=\frac{1}{K_{12} R_{1}}\left(-Q_{2} K_{12}\right)=-\frac{Q_{2}}{R_{1}} \\
& T_{2}=\frac{1}{K_{12} R_{1}}\left(-Q_{2}\left(K_{12}-R_{1}\right)\right)=T_{R_{2}}
\end{aligned}
$$

Como se ve la temperatura $T_{2}=T_{R_{2}}$, por lo tanto conseguimos un error de cero en este nodo. En cambio la temperatura $T_{1}$ no es posible ajustarla empleando este parámetro, como se ve en el jacobiano (2.23) ya que $\partial T_{1} / \partial K_{12}=0$. 


\subsection{Actualización de modelos}

El procedimiento que se presenta en este apartado se puede aplicar en el caso en que se pretenda realizar pequeños ajustes en los valores de los parámetros para conseguir que el modelo matemático reproduzca las temperaturas de referencia. Este método se puede aplicar porque los valores de las temperaturas en el modelo de partida están cercanos a la temperatura de referencia, siempre que se cumplan las condiciones necesarias, como es el caso del ajuste de un TMM a temperaturas de referencia.

Se ha explicado en el apartado 2 la forma en que en un entorno máximo se pueden expresar las fluctuaciones de temperatura respecto a un punto inicial debido a las fluctuaciones de las propiedades. En algún lugar dentro de este pequeño entorno alrededor del punto inicial se encuentra los valores de los parámetros de referencia (si estamos en el espacio de búsqueda) o de las temperaturas de referencia (si nos fijamos en el espacio de las temperaturas).

Lo que en definitiva se va a hacer es tratar de, mediante pequeños ajustes en los parámetros $\boldsymbol{X}$, obtener unas temperaturas estimadas, $\boldsymbol{T}$, lo más cercanas posibles a $T_{R}$

Dado que se van a hacer pequeños cambios, se puede linealizar la relación $\boldsymbol{T}(\boldsymbol{X})$ alrededor de la solución inicial $\boldsymbol{T}^{\mathbf{0}}$ que hemos obtenido usando el conjunto de parámetros iniciales $\boldsymbol{X}^{\mathbf{0}}$ como ya se hizo en (2.14),

$$
\boldsymbol{T}=\boldsymbol{T}^{\mathbf{0}}+\sum_{h}^{N p} \frac{\partial \boldsymbol{t}}{\partial x_{h}} x_{h}
$$

La condición de mínimo error (2.5) para cada parámetro, $X_{i}$, viene dada por:

$$
\sum_{j=1}^{N_{N}}\left(T_{R_{j}}-T_{j}\right) \frac{\partial t_{j}}{\partial x_{i}}=0
$$


utilizando (2.30) se tiene

$$
\sum_{j=1}^{N_{N}}\left(T_{R_{j}}-T_{j}^{0}-\sum_{h=1}^{N_{P}} \frac{\partial t_{j}}{\partial x_{h}} x_{h}\right) \frac{\partial t_{j}}{\partial x_{i}}=0
$$

Tomando $t_{R_{j}}=T_{R_{j}}-T_{j}^{0}$ como la fluctuación de las temperaturas iniciales estimadas respecto a las temperaturas de referencia, se reescribe esta expresión como

$$
\sum_{j=1}^{N_{N}}\left(t_{R_{j}}-\sum_{h=1}^{N_{P}} \frac{\partial t_{j}}{\partial x_{h}} x_{h}\right) \frac{\partial t_{j}}{\partial x_{i}}=0 \quad ; \quad i=1 \ldots N_{P}
$$

En la expresión (2.33) se identifican dos sumandos, que se van a tratar por separado. El primer sumando,

$$
\sum_{j=1}^{N_{N}} t_{R_{j}} \frac{\partial t_{j}}{\partial x_{i}}=t_{R_{1}} \frac{\partial t_{1}}{\partial x_{i}}+t_{R_{2}} \frac{\partial t_{2}}{\partial x_{i}}+\cdots=\left.\boldsymbol{t}_{\boldsymbol{R}}^{T} \mathbf{M}\right|_{\text {columna } i}
$$

se corresponde con la multiplicación del vector de las temperaturas de referencia con cada columna de la matriz jacobiana, $\mathbf{M}$, y se puede escribir como $\mathbf{M}^{T} \boldsymbol{t}_{\boldsymbol{R}}$. El segundo sumando se puede reescribir

$$
\sum_{j=1}^{N_{N}}\left(\sum_{h=1}^{N_{P}} \frac{\partial t_{j}}{\partial x_{h}} x_{h}\right) \frac{\partial t_{j}}{\partial x_{i}}=\sum_{j=1}^{N_{N}} \gamma_{j} \frac{\partial t_{j}}{\partial x_{i}}=\left.\gamma_{j}^{T} \mathbf{M}\right|_{\text {columna } i}
$$

donde se ha escrito $\gamma_{j}=\sum_{h=1}^{N_{P}} \frac{\partial t_{j}}{\partial x_{h}} x_{h}=\left.\mathbf{M}\right|_{f i l a}{ }_{j} \boldsymbol{x}$, vector columna que combinado con (2.35) queda:

$$
\gamma_{j} \frac{\partial t_{j}}{\partial x_{i}}=\gamma^{T} \mathbf{M}=(\mathbf{M} \boldsymbol{x})^{T} \mathbf{M}=\boldsymbol{x}^{T} \mathbf{M}^{T} \mathbf{M}
$$

Agrupando de nuevo los dos sumandos de la ecuación (2.33), condición de mínimo error se llega a

$$
\mathbf{M}^{T} \boldsymbol{t}_{\boldsymbol{R}}-\left(\boldsymbol{x}^{T} \mathbf{M}^{T} \mathbf{M}\right)^{T}=0
$$


y reordenando la igualdad, se obtiene

$$
\mathbf{M}^{T} \boldsymbol{t}_{\boldsymbol{R}}=\left(\boldsymbol{x}^{T} \mathbf{M}^{T} \mathbf{M}\right)^{T}
$$

Finalmente, haciendo la traspuesta del lado derecho, se obtiene un sistema con $N_{P}$ ecuaciones para $\boldsymbol{x}$

$$
\mathbf{M}^{T} \boldsymbol{t}_{\boldsymbol{R}}=\mathbf{M}^{T} \mathbf{M} \boldsymbol{x}
$$

Para poder resolver este sistema se multiplica (2.39) por la izquierda utilizando la inversa de $\mathbf{M}_{\mathbf{c}}=\mathbf{M}^{T} \mathbf{M}$ si $\mathbf{M}$ no es singular ${ }^{2}$.

La solución $\boldsymbol{x}^{*}$, a este sistema de ecuaciones vendrá dada por [46]:

$$
\boldsymbol{x}^{*}=\mathbf{M}^{+} \boldsymbol{t}_{\boldsymbol{R}} \quad \text { dónde } \quad \mathbf{M}^{+}=\left(\mathbf{M}^{T} \mathbf{M}\right)^{-1} \mathbf{M}^{T}
$$

$\mathbf{M}^{+}$coincide con la pseudoinversa de $\mathbf{M}$ (inversa de Moore-Penrose) en el caso que $\mathbf{M}$ esté compuesta por columnas linealmente independientes. De esta manera se puede calcular $\boldsymbol{x}$ para conseguir $\boldsymbol{t}_{\boldsymbol{R}}$. En este sistema de ecuaciones, se pueden distinguir dos casos:

- Si $N_{P}>N_{N}$, tendremos una matriz $\mathbf{M}_{\mathbf{c}}$ de rango $N_{N}$ como máximo, lo que proporciona un sistema con infinitas soluciones (sistema indeterminado). Este caso no es deseable hacer el ajuste. El modelo ajustado no tiene porque coincidir con la realidad aunque proporcione los resultados deseados.

- Si $N_{P}<N_{N}$, la matriz $\boldsymbol{M}_{\boldsymbol{c}}$ tendrá como máximo de rango $N_{P}$, y se tiene un sistema sobredeterminado sin una solución en el caso general. Este caso es el interesante porque se puede encontrar una solución aproximada que mejor se ajuste a los resultados de referencia. Para esto se puede hacer uso del método de la pseudo-inversa [49].

\footnotetext{
${ }^{2}$ La matriz $\mathbf{M}_{\mathbf{c}}$ es una matriz cuadrada simétrica de dimensión $N_{P} \times N_{P}$.
} 


\subsection{Generalización a varios casos de carga}

Como se ha explicado en el apartado 1.1.2, generalmente en los modelos térmicos matemáticos se estudian varios casos de carga diferentes correspondientes a distintos modos de funcionamiento o escenarios que el satélite puede encontrar en órbita. Por este motivo cuando se dispone de temperaturas de referencia para actualizar un modelo, normalmente se dispondrá de varios conjuntos de temperaturas de referencia, uno por caso de carga. Como en el método de ajuste presentado en 2.4 solo ha tenido en cuenta el ajuste para un caso de carga, es necesario generalizar el resultado encontrado en (2.40) de manera que se pueda aplicar a varios casos de carga. Cada uno de ellos llevará asociada una matriz $\mathbf{M}$ de sensibilidades, como se ha deducido en (2.10).

Como demostración de cómo se generaliza a varios casos de carga, primero se van a considerar dos casos que se van a denominar como $C_{1}$ y $C_{2}$. Siguiendo la misma línea que con estos dos casos se puede observar como una generalización a $N_{C}$ casos de carga es trivial.

Partiendo de (2.31), se puede escribir la condición de mínimo error para los casos $C_{1}$ y $C_{2}$ como

$$
\left.\sum_{j=1}^{N_{N}}\left(T_{R_{j}}-T_{j}\right) \frac{\partial t_{j}}{\partial x_{i}}\right|_{C_{1}}+\left.\sum_{j=1}^{N_{N}}\left(T_{R_{j}}-T_{j}\right) \frac{\partial t_{j}}{\partial x_{i}}\right|_{C_{2}}=0
$$

Para cada uno de los dos términos (uno por caso de carga) se puede seguir el mismo procedimiento que se ha empleado para llegar a (2.39),

$$
\left.\mathbf{M}^{T} \boldsymbol{t}_{\boldsymbol{R}}\right|_{C_{1}}+\left.\mathbf{M}^{T} \boldsymbol{t}_{\boldsymbol{R}}\right|_{C_{2}}=\left.\mathbf{M}^{T} \mathbf{M} \boldsymbol{x}\right|_{C_{1}}+\left.\mathbf{M}^{T} \mathbf{M} \boldsymbol{x}\right|_{C_{2}}
$$

Como el vector $\boldsymbol{x}$ es el mismo en los dos casos de carga, ya que lo que se busca es el conjunto de parámetros $X$ que mejor ajusta todos los casos de carga, se pueden agrupar ambos sumandos, de manera que

$$
\left.\mathbf{M}^{T} \boldsymbol{t}_{\boldsymbol{R}}\right|_{C_{1}}+\left.\mathbf{M}^{T} \boldsymbol{t}_{\boldsymbol{R}}\right|_{C_{2}}=\left(\left.\mathbf{M}^{T} \mathbf{M}\right|_{C_{1}}+\left.\mathbf{M}^{T} \mathbf{M}\right|_{C_{2}}\right) \boldsymbol{x}
$$


Finalmente, y de manera análoga a (2.40), se obtiene:

$$
\boldsymbol{x}^{*}=\mathbf{M}_{\mathbf{c}{ }^{-1} C_{2}}^{-1}\left(\left.\mathbf{M}^{T} \boldsymbol{t}_{\boldsymbol{R}}\right|_{C_{1}}+\left.\mathbf{M}^{T} \boldsymbol{t}_{\boldsymbol{R}}\right|_{C_{2}}\right)
$$

donde se han combinado las dos matrices de sensibilidades de los casos $\mathbf{M}_{C_{1}}$ y $\mathbf{M}_{C_{2}}$ en la matriz cuadrada $\mathbf{M}_{\mathbf{c} C_{1} C_{2}}$

$$
\mathbf{M}_{\mathbf{c} C_{1} C_{2}}=\left.\mathbf{M}^{T} \mathbf{M}\right|_{C_{1}}+\left.\mathbf{M}^{T} \mathbf{M}\right|_{C_{2}}
$$

Como se puede observar de manera similar se pueden añadir cuantos casos de carga sea necesario para la correlación. En cierta manera se puede interpretar la expresión (2.44) como la influencia de cada caso en la corrección de los parámetros $\boldsymbol{x}$.

Generalizando a $N_{C}$ casos de carga, se puede obtener la corrección en los parámetros como:

$$
\boldsymbol{x}^{*}=\left.\mathbf{M}_{\mathbf{c}}^{-\mathbf{1}} \sum_{k=1}^{N_{C}}\left(\mathbf{M}^{T} \boldsymbol{t}_{\boldsymbol{R}}\right)\right|_{C_{k}}
$$

donde $\mathbf{M}_{\mathbf{c}}=\left.\sum_{k=1}^{N_{C}}\left(\mathbf{M}^{T} \mathbf{M}\right)\right|_{C_{k}}$.

Si bien la solución presentada en (2.40) para un caso de carga (es decir, para un jacobiano fijo) es conocida, la expresión (2.46) para múltiples casos de carga es una de las contribuciones de esta tesis. 


\section{Capítulo 3}

\section{Ajuste de modelos térmicos matemáticos}

Como se ha presentado en el apartado 2, partiendo de una función objetivo que minimizar (2.2) es posible obtener a una expresión general para calcular la variación que hay que introducir en los parámetros del modelo para que éste proporcione una estimación los más ajustada posible a las temperaturas de referencia.

En este apartado se presenta la implementación práctica realizada de este método. Como se ha indicado en el apartado 1.3, uno de los objetivos principales de la tesis es la integración del método descrito en el trabajo del ingeniero de control térmico. Para ello se han desarrollado las herramientas necesarias de tal manera que el ajuste del modelo se pueda realizar con facilidad utilizando directamente los archivos que genera el software ESATAN-TMS [7].

Además, primero se explicarán una serie de requisitos que se han de cumplir para que el ajuste del modelo sea exitoso.

\subsection{Requisitos}

El método propuesto está basado en el cálculo de la matriz de sensibilidades o matriz jacobiana. Para la obtención de soluciones se necesita que esta matriz no sea singular y esto impone una serie de requisitos. Siguiendo la linea descrita en [36] se 
va a definir un conjunto de requisitos que se han de cumplir para poder aplicar el método.

\subsubsection{Características del modelo matemático}

Una limitación del método propuesto es que cada temperatura obtenida a través del modelo debe ser una función monótona y diferenciable respecto a los parámetros. Estas condiciones en general se cumplen en el espacio de soluciones de un TMM, salvo en ciertas regiones que merecen un estudio aparte.

En las misiones espaciales las condiciones de contorno son muy extremas y a menudo se recurre a dispositivos tales como heaters ${ }^{1}$, heat pipes. ${ }^{2}$, TECs ${ }^{3}$, etc., que no presentan un comportamiento diferenciable y monótono. Afortunadamente, solo se requiere este comportamiento en un entorno del espacio de las soluciones muy limitado, en el entorno que se va a aplicar el ajuste.

De manera muy sencilla vamos a ver a qué nos referimos con comportamiento de la función de la temperatura respecto a los parámetros. La Figura 3-1 muestra el comportamiento de cierta temperatura, $T_{n}$, respecto a un parámetro $X_{m}$. Podemos distinguir 4 zonas de interés. En la primera zona el comportamiento no es monótono y encontramos un mínimo, en la zona 3 entra en juego algún mecanismo de control y la función deja de ser diferenciable y en la zona 4 ya deja de ser dependiente del parámetro. La única zona de interés y donde el método se puede aplicar es la zona 2.

Si intentásemos ajustar las temperaturas en el entorno de la zona 1 o 3, el método propuesto no convergería y probablemente no se podrían encontrar resultados satisfactorios. El caso de la zona 4 está contemplado en el requisito del apartado 3.1.2.

\subsubsection{Observabilidad de los parámetros}

Es importante que los parámetros seleccionados para el ajuste del modelo tengan influencia en al menos una temperatura. Si se da el caso, por ejemplo de la zona 4

\footnotetext{
${ }^{1}$ Calentadores.

${ }^{2}$ Caloducto.

${ }^{3}$ Termoelectric Cooler, enfriador por efecto Peltier.
} 


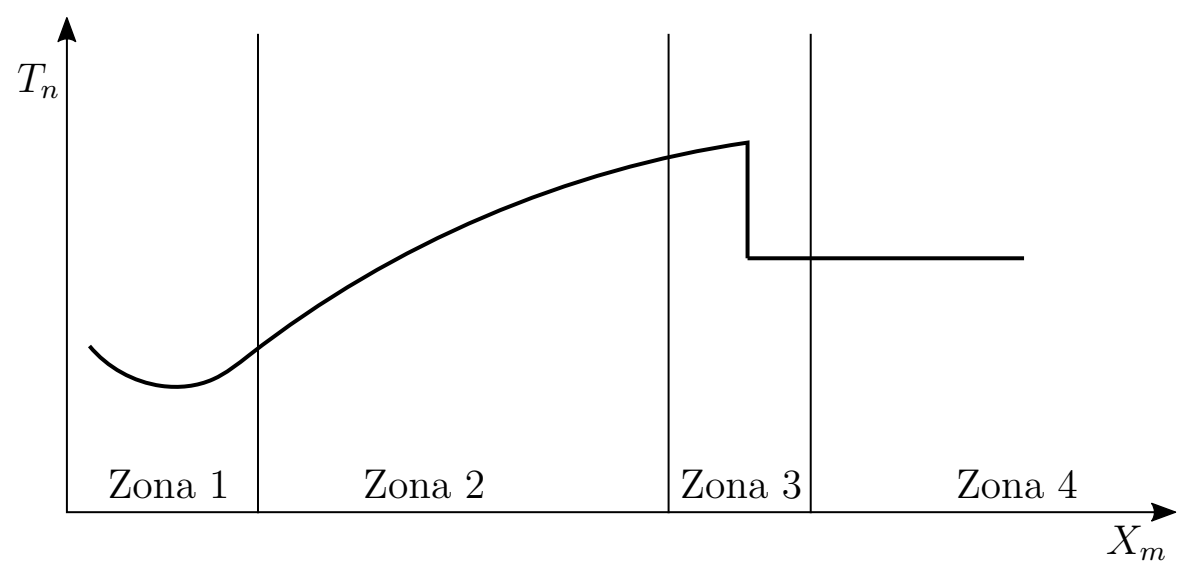

Figura 3-1: Dependencia de la temperatura $T_{n}$ respecto al parámetro $X_{m}$.

indicada en la Figura 3-1 de manera que el parámetro tenga muy poca influencia o nula en las temperaturas del modelo, aparecerá una columna de valores nulos o muy pequeños en el jacobiano, lo que significa que ese parámetro no se puede ajustar, es decir, no tiene sentido emplearlo en el proceso.

Otro punto muy importante es la unicidad en la respuesta de los parámetros. Si dos parámetros diferentes producen el mismo efecto (o muy parecido) será imposible ajustar el modelo correctamente y habrá que desechar uno de los parámetros. Esta situación genera dos columnas casi idénticas en la matriz de sensibilidades y al igual que en el caso anterior generará problemas e inestabilidades a la hora de calcular los parámetros [50,51]. El significado es que en realidad el efecto de los parámetros es el mismo, por lo que la elección de considerarlos por separado no ha sido correcta, al menos para el caso de carga considerado. La matriz $\mathbf{M}$ no debe tener una columna de ceros o dos o más columnas linealmente dependientes.

Esto es especialmente interesante y ha de ser tenido en cuenta si queremos diseñar un test específico para ajustar el modelo. La manera más clara es por tanto calcular previamente la matriz de sensibilidades y observar que parámetros tienen efecto en el modelo. 


\subsubsection{Observabilidad de los resultados}

Al igual que con los parámetros, todas las temperaturas seleccionadas para ajustar deben de estar influenciadas por al menos un parámetro. Si estamos teniendo en cuenta una temperatura que no se ve afectada (o muy poco) por ningún parámetro, tendremos una fila de valores nulos o muy pequeños en la matriz $\mathbf{M}$.

Al igual que el caso anterior, si dos temperaturas se ven igualmente afectadas por los parámetros, tendremos un jacobiano pobremente condicionado. En este caso se estarían considerando dos nodos tan unidos térmicamente que no serían distinguibles en el modelo, por lo que deberían condensarse en uno solo, reduciendo así el tamaño del modelo.

\subsubsection{Elección e incertidumbre de las temperaturas de refe- rencia}

El objetivo del proceso es ajustar el modelo matemático de manera que las temperaturas obtenidas sean lo más parecido posible a las temperaturas que se toman de referencia. Estas temperaturas de referencia no siempre cubren todos los nodos del modelo. En el caso de actualizar un modelo respecto a temperaturas obtenidas en un ensayo, solo pueden ajustarse los puntos donde se colocaron los sensores de temperatura. Es por lo tanto necesario seleccionar correctamente los puntos que se van a usar en el ajuste.

Hay que tener en cuenta la incertidumbre en las temperaturas de referencia. Esto es especialmente importante si se trata de datos experimentales. No tiene sentido ajustar el modelo con unas temperaturas de referencia que tengan asociadas una incertidumbre mayor al máximo error buscado. Se puede dar el caso de que el error en la medida no permita ajustar el modelo ya que este no sea capaz de reproducirlas.

De esta forma, del análisis de condicionamiento del jacobiano es posible sacar conclusiones sobre la selección de los parámetros a ajustar y sobre los puntos de referencia de la temperatura, para el caso de carga considerado, permitiendo reducir el tamaño del modelo (en nodos y parámetros) así como evaluar la idoneidad de la 
posición de los nodos seleccionados.

\subsection{Algoritmo GIPI}

El algoritmo desarrollado, GIPI, de sus siglas en inglés Generalized Iterative Pseudoinverse, está esquematizado en la Figura 3-2. El método ha sido implementado en Python [52] utilizando librerías de SciPy [53], Numpy [54] y Matplotlib [55] para generar los gráficos. Se comenta a continuación el procedimiento seguido.

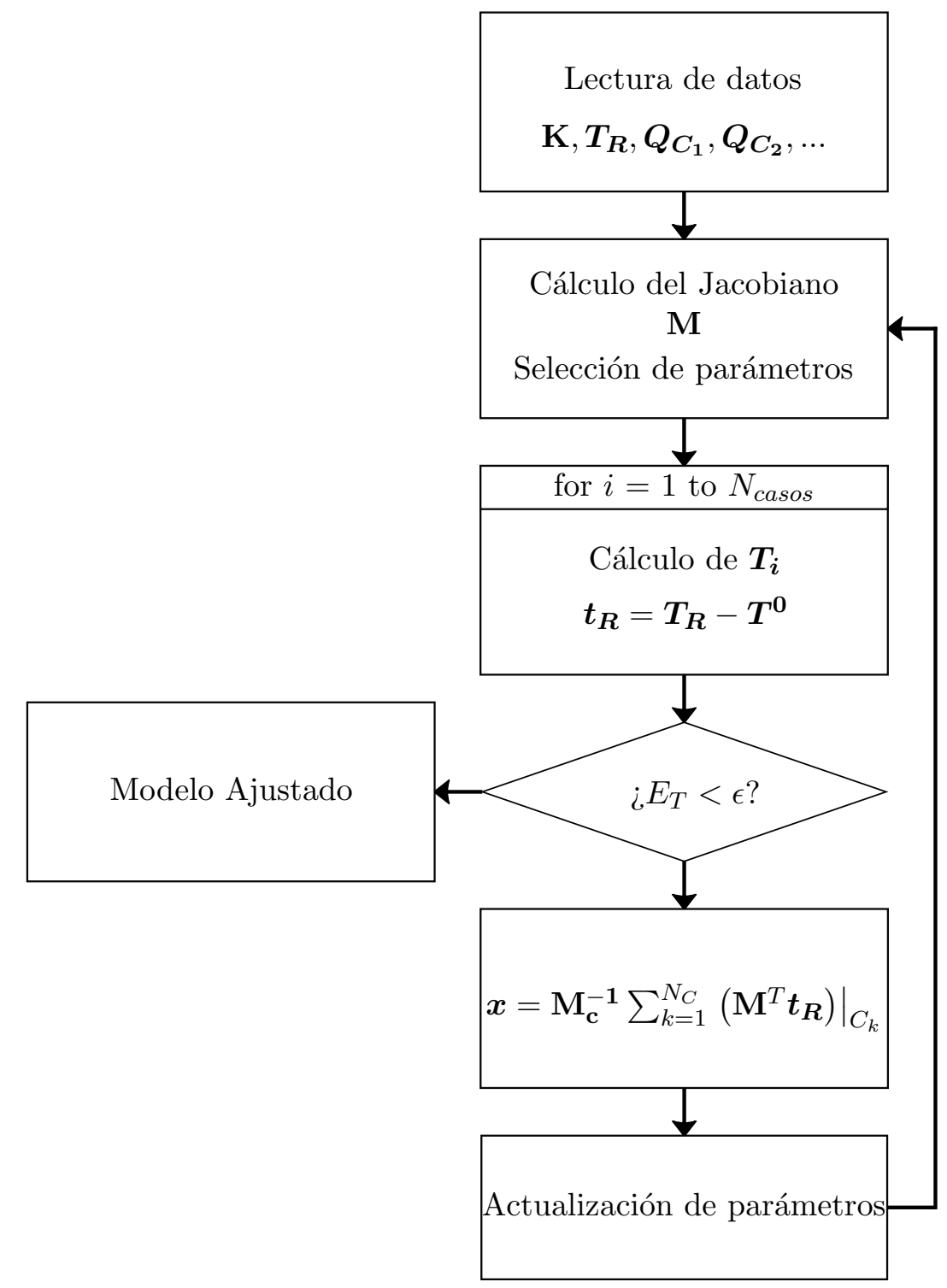

Figura 3-2: Diagrama del flujo del algoritmo de implementación del método. 


\subsubsection{Lectura de los datos}

Con la intención de que este método sea útil y se pueda aplicar en proyectos futuros de una manera sencilla, se ha buscado la compatibilidad con ESATAN-TMS. De esta manera, la lectura de los datos del modelo matemático se hace a través de los archivos .TMD. Estos archivos binarios los genera ESATAN-TMS (de manera opcional) como parte de la solución de un caso de carga. De manera que, es necesario conservar un archivo .TMD por cada caso de carga que se quiera usar para ajustar el modelo.

Los archivos .TMD contienen toda la información necesaria para la ejecución de un TMM ordenada siguiendo la estructura HDF5 [56]. Con la información que se obtiene de cada archivo se construyen las matrices de acoplamientos lineales, $\mathbf{K}$, radiativos, $\mathbf{R}$ y los vectores de carga $\boldsymbol{Q}$ y capacidades térmicas $\boldsymbol{C}$. De manera que se dispone de toda la información para poder resolver el modelo (1.1) y calcular las temperaturas estimadas por los parámetros.

Las temperaturas de referencia $\boldsymbol{T}_{\boldsymbol{R}}$ pueden venir de fuentes diferentes, ya sean temperaturas medidas en un ensayo u obtenidas mediante otro modelo, por ejemplo, un detallado, DTMM. Estas $\boldsymbol{T}_{\boldsymbol{R}}$ hay que proporcionarlas aparte en una lista ordenada, de manera que coincida con el orden de la numeración de los nodos del modelo.

En general, los parámetros que se van a variar manifiestan su influencia a través de los elementos de la matriz $\mathbf{K}$, de conductancias térmicas. En el estado en que está la aplicación del método, solo se ha considerado la variación de los valores de estas conductancias directamente, no las variables físicas originales de las que estas dependen. En principio todos los elementos de esta matriz son parámetros candidatos para el ajuste, aunque hay que estudiar con detenimiento cuáles deben emplearse en el ajuste, como se explica a continuación. 


\subsubsection{Selección de los parámetros y nodos del modelo}

Para ayudar a seleccionar los parámetros adecuados para realizar la actualización de un modelo se va a definir la matriz de influencia, $\mathbf{I}_{\mathbf{X}}$,

$$
\mathbf{I}_{\mathbf{X}}=\left[\begin{array}{cccc}
\frac{\partial T_{1}}{\partial X_{1}} \delta X_{1} & \frac{\partial T_{1}}{\partial X_{2}} \delta X_{2} & \ldots & \frac{\partial T_{1}}{\partial X_{N_{P}}} \delta X_{N_{P}} \\
\ldots & \ldots & & \ldots \\
\frac{\partial T_{N_{N}}}{\partial X_{1}} \delta X_{1} & \frac{\partial T_{N_{N}}}{\partial X_{2}} \delta X_{2} & \ldots & \frac{\partial T_{N_{N}}}{\partial X_{N_{P}}} \delta X_{N_{P}}
\end{array}\right]=\mathbf{M} \delta \boldsymbol{X}
$$

donde $\mathbf{M}$ es el jacobiano y $\delta \boldsymbol{X}$ es un vector cuyos elementos son las variaciones admisibles dentro del diseño concreto que se esté actualizando. En la matrix de influencia $\mathbf{I}_{\mathbf{X}}$ cada columna representa la variación en temperatura de los nodos que es generada por una variación en el parámetro, $\delta X_{i}$. Los elementos de esta matriz tienen por tanto, dimensiones de temperatura y dan una idea del efecto que produce la variación de cada parámetro del modelo. Esta comparación entre el valor de los distintos elementos no es posible hacerla directamente empleando el jacobiano M. En el estudio siguiente consideramos $\delta X_{i}=0,1 X_{i}$.

Con la ayuda de esta matriz se puede identificar qué parámetros no tienen un efecto significativo en las temperaturas del modelo. De igual manera se puede ver qué nodos no se ven afectados por ningún parámetro.

Si se va a intentar correlacionar un modelo respecto unas temperaturas de referencia procedentes de un ensayo es probable si no se ha diseñado el ensayo adecuadamente que no se disponga del valor de la temperatura de referencia de todos los nodos. Por lo tanto el tamaño máximo del vector de referencia, $\boldsymbol{T}_{\boldsymbol{R}}$, es también el máximo número de nodos que pueden tenerse en cuenta en la correlación.

Para completar la selección de los parámetros es muy útil disponer de la matriz de sensibilidad del modelo, M. Da la información de qué parámetros son linealmente dependientes y por lo tanto hay que omitirlos (o expresarlos de otra manera) para el ajuste del modelo. Para ello se va a tener en cuenta tanto el rango de la matriz como el parámetro de condición. Este número informa de lo sensible que será la salida de una función respecto a una pequeña variación en los parámetros [57]. Es importante 
obtener una matriz jacobiana bien condicionada ya que más tarde puede dar lugar a inestabilidades durante el proceso de ajuste.

El cálculo de la matriz jacobiana $\mathbf{M}$, se hace numéricamente, empleando el método de la derivada hacia adelante, con dos puntos. Se varía cada parámetros individualmente y se calculan las temperaturas del modelo, y a partir de estas temperaturas se obtiene $\Delta T_{i}$ que dividiremos por la variación del parámetro. La variación que efectuamos en cada parámetro para este cálculo es de un $10 \%$ de su valor.

\subsubsection{Ajuste de parámetros}

Una vez se ha establecido que el error inicial entre el modelo actual y el de influencia supera los límites requeridos y se concluye que es necesario realizar una actualización del modelo ajustando sus parámetros, se calcula la corrección necesaria a cada parámetro empleando (2.46) y generando un nuevo conjunto de parámetros. A partir de estos nuevos valores se puede determinar el error del ajuste. En caso de no ser satisfactorio, se puede emplear el algoritmo de forma recurrente.

Esta actualización de parámetros hay que hacerla con cuidado porque los parámetros representan magnitudes físicas y sus valores están sujetos a ciertas limitaciones. Por ejemplo la conductividad térmica de un material no puede ser negativa y además debe mantenerse dentro de unos valores que se correspondan a las características reales del material. Para asegurar esto hay que establecer limitaciones, definidas co$\operatorname{mo} X_{i}^{\max }$ y $X_{i}^{\min }$.

En el caso que se esté actualizando un modelo matemático reducido respecto de un modelo detallado hay que tener en cuenta que los parámetros serán casi siempre los acoplamientos lineales y que éstos pueden tener cualquier valor positivo en general. Aún así es recomendable establecer ciertos límites ya que pueden existir problemas de convergencia (en cálculos transitorios) si los acoplamientos térmicos de un nodo son muy dispares [58].

Por ello, se ha establecido una tasa máxima de variación del parámetro para evitar que se produzcan variaciones muy fuertes, limitando la variación del parámetro a un porcentaje de su valor. Hay que tener en cuenta que se ha supuesto que la 
solución está en un entorno pequeño en el espacio de búsqueda cercano a $X_{R}$ y que el comportamiento de la solución en dicho entorno es lineal y monótono. Limitando esta tasa de variación del parámetro se impide que el valor de los parámetros salga de este entorno.

En adelante esta tasa se la denomina coeficiente de variación máxima, $c_{v}$ y podrá tener un valor positivo. El comportamiento de la convergencia del método depende de este valor. La influencia de este coeficiente en el caso concreto del ajuste de los parámetros de PHI O-Unit se presenta en el apartado 5.3.4.

Un coeficiente de variación $c_{v}=0,5$ indica que el parámetro solo puede variar un $50 \%$ en cada iteración. Este valor ha sido encontrado experimentalmente en las pruebas realizadas, y ha mostrado un buen comportamiento en la mayoría de los casos. Se puede considerar como un buen punto de partida.

El $c_{v}$ óptimo supone un compromiso entre el número de iteraciones necesarias y la oscilación en las soluciones. Un valor más seguro es limitar la variación máxima a un $10 \%$, que es lo utilizado para el cálculo del jacobiano. Pero esto proporcionará una convergencia más lenta.

En la Figura 3-3 se presenta el algoritmo de actualización de los parámetros. Una vez calculada la variación a introducir en el valor del parámetro, $x_{i}$, se pueden seguir dos vías dependiendo de si esta variación es positiva o negativa.

- Si $x_{i}$ es negativo hay que tener cuidado porque el valor del parámetro, $X_{i}$ podría hacerse negativo. Primero se calcula la variación máxima que puede tomar el parámetro, $x_{i}^{\max }$ de acuerdo al coeficiente de variación máxima, $c_{v}$. Luego se comprueba la variación del parámetro es mayor que esta variación máxima, y si es así entonces se limita su valor a $x_{i}^{\max }$. En caso contrario se deja como estaba. Lo siguiente que se comprueba es que el parámetro no supere el límite inferior $X_{i}^{m i n}$. Por defecto se comprueba que el parámetro nunca sea negativo, pero este límite inferior podría ser cualquier otro valor.

- Si $x_{i}$ es positivo, se hace una comprobación similar. Lo primero que comprueba es que la variación máxima no supere la impuesta por $c_{v}$ y también se comprueba que el valor del parámetro $X_{i}$, no supere el límite superior $X_{i}^{\max }$ establecido. 
La limitación superior previene que el valor del parámetro crezca sin control.

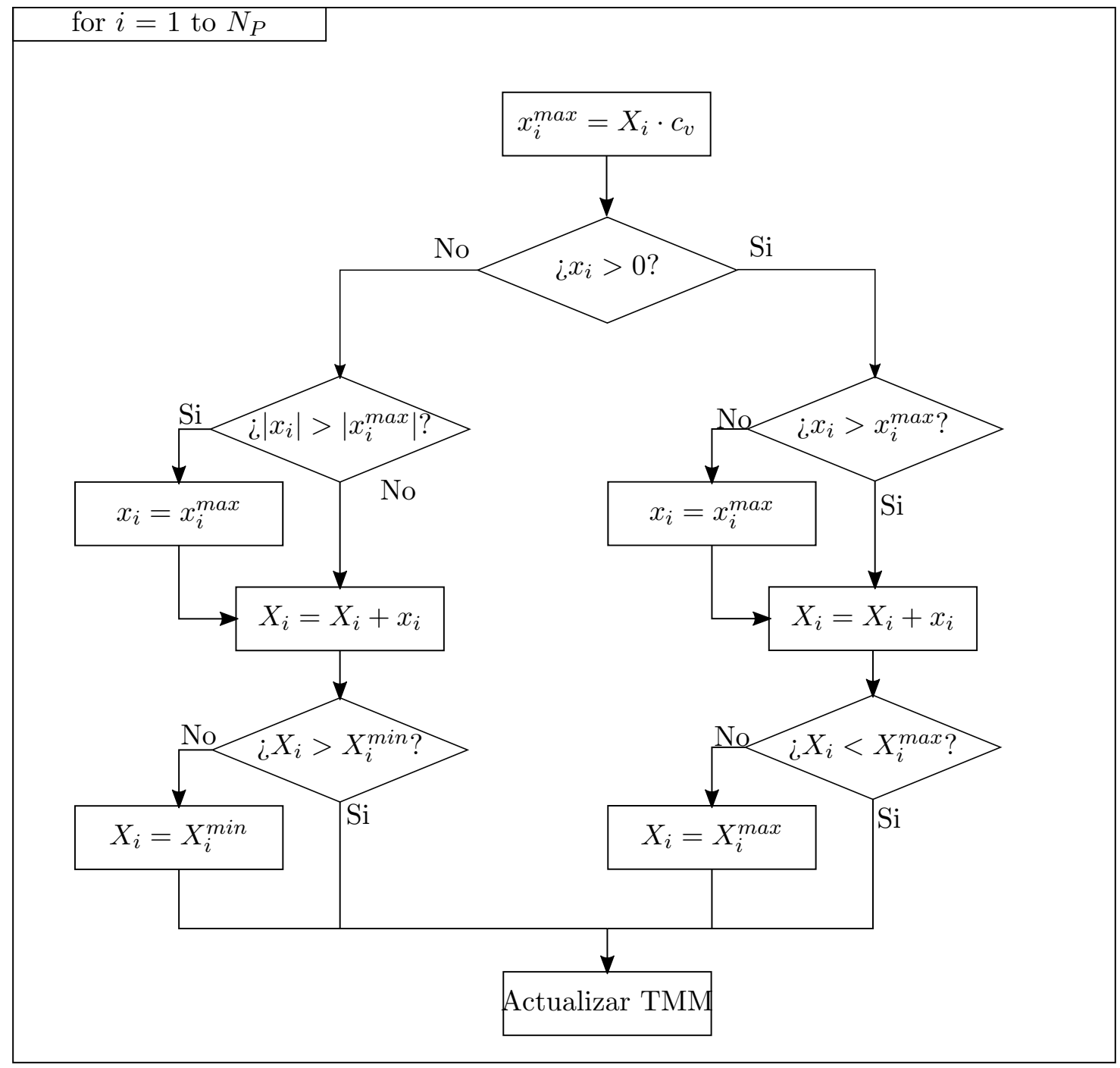

Figura 3-3: Diagrama de flujo del algoritmo de actualización parámetros.

Por otra parte, la implementación realizada del método permite entre elegir dos tipos de parada: por número de iteraciones y por evolución del RSS, definido en el apartado 2.1. Si se usa la segunda opción las iteraciones se detendrán cuando se observe un crecimiento del RSS. En algunos esto puede dar lugar a una parada prematura ya que en algunos modelos se ha observado como el RSS pasa por un mínimo local durante las iteraciones. 


\section{Capítulo 4}

\section{Modelo 4 nodos}

Para ayudar a explicar el funcionamiento del método GIPI se ha aplicado primero a un modelo simple de 4 nodos. De esta manera se pueden analizar diferentes situaciones manteniendo un número manejable de parámetros. El modelo que se va a utilizar está basado en el descrito en [36]. Como se muestra en [15], el modelo propuesto es interesante para este tipo de estudios, pero los casos de carga propuestos no son los más idóneos. A diferencia de éste, en el que se aplicaba la misma carga $10 \mathrm{~W}$ en los cuatro nodos, se han aplicado diferentes cargas en los diferentes nodos con el fin de evitar la simetría de las cargas y el consecuente mal condicionamiento en la matriz jacobiana, $\mathbf{M}$.

El modelo (Fig. 4-1) consta de 4 nodos conectados entre ellos mediante conductancias lineales, $\left(G_{L} \mathrm{~S}\right.$, usando la nomenclatura de ESATAN-TMS) y con el entorno a través de conductacias radiativas $\left(G_{R} \mathrm{~s}\right)$. El nodo 1 además está conectado también conductivamente al entorno. Cada uno de los nodos disipa una potencia diferente $\left(Q_{1}=10 \mathrm{~W}, Q_{2}=8 \mathrm{~W}, Q_{3}=12 \mathrm{~W}\right.$ y $\left.Q_{4}=9 \mathrm{~W}\right)$.

Para el análisis de este modelo se supone que los valores de los $G_{R i}$ son fijos y tienen todos el mismo valor, $G_{R i}=0.1 \mathrm{~m}^{2}$. Tanto la temperatura del entorno radiativo como conductivo está fijada a $T_{B}=0{ }^{\circ} \mathrm{C}$ y el $G_{L_{1} B}$ no puede variar, teniendo un valor de $G_{L 1 B}=1.0 \mathrm{~W} \mathrm{~K}^{-1}$. Cada $G_{L i j}$ entre los nodos representa un parámetro de ajuste del modelo.

Aplicando la ecuación de balance energético a cada nodo, se obtiene el siguiente 


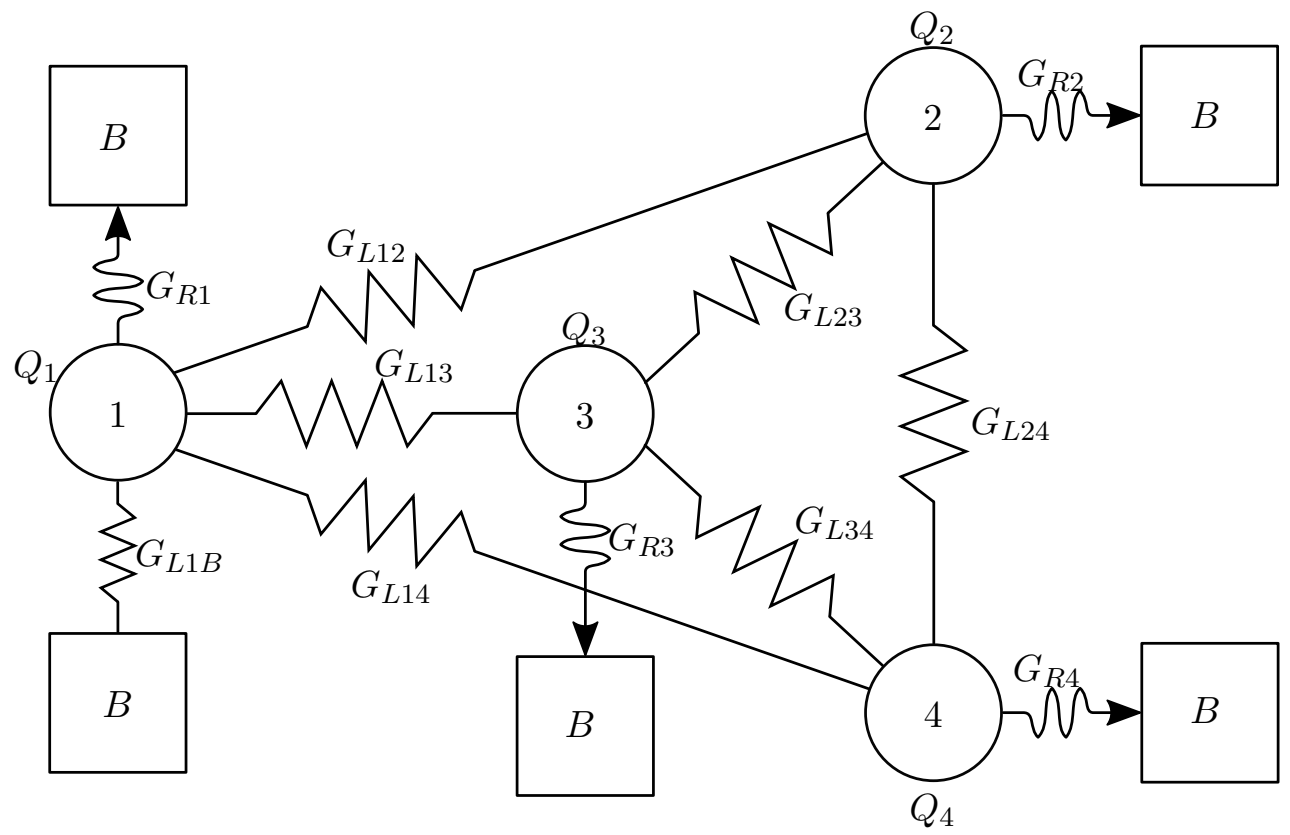

Figura 4-1: Esquema del modelo de 4 nodos empleado [36].

sistema de ecuaciones:

$$
\begin{array}{r}
G_{L 12}\left(T_{2}-T_{1}\right)+G_{L 13}\left(T_{3}-T_{1}\right)+G_{L 14}\left(T_{4}-T_{1}\right)+G_{L 1 B}\left(T_{B}-T_{1}\right)+ \\
+\sigma G_{R 1}\left(T_{B}^{4}-T_{1}^{4}\right)+Q_{1}=0 \\
G_{L 12}\left(T_{1}-T_{2}\right)+G_{L 23}\left(T_{3}-T_{2}\right)+G_{L 24}\left(T_{4}-T_{2}\right)+\sigma G_{R 2}\left(T_{B}^{4}-T_{2}^{4}\right)+Q_{2}=0 \\
G_{L 13}\left(T_{1}-T_{3}\right)+G_{L 23}\left(T_{2}-T_{3}\right)+G_{L 34}\left(T_{4}-T_{3}\right)+\sigma G_{R 3}\left(T_{B}^{4}-T_{3}^{4}\right)+Q_{3}=0 \\
G_{L 14}\left(T_{1}-T_{4}\right)+G_{L 24}\left(T_{2}-T_{4}\right)+G_{L 34}\left(T_{3}-T_{4}\right)+\sigma G_{R 4}\left(T_{B}^{4}-T_{4}^{4}\right)+Q_{4}=0
\end{array}
$$

Las temperaturas de referencia de este modelo, mostradas en la Tabla 4.1, se han obtenido empleando un conjunto de parámetros, $X_{R}$ (establecido arbitrariamente) y resolviendo el sistema de ecuaciones (4.1). Estas temperaturas corresponderían a las medidas de un ensayo o al resultado de otro modelo, en general detallado, y el objetivo del ajuste es obtenerlas mediante el modelo. En el análisis de este modelo térmico de 4 nodos se supone que cada $G_{L i j}$ es un parámetro de modelo. La Tabla 4.2 muestra esta relación entre los $G_{L i j}$ y los parámetros del modelo $X_{i}$, así como su valor. Usando este conjunto de parámetros de referencia, $X_{R}$, se obtienen las temperaturas 
de referencia, $T_{R}$.

Tabla 4.1: Modelo de 4 nodos. Temperaturas de referencia, $T_{R}$.

\begin{tabular}{|c|c|}
\hline Nodo & Temperatura $\left[{ }^{\circ} \mathrm{C}\right]$ \\
\hline 1 & 8.76 \\
2 & 15.62 \\
3 & 19.15 \\
4 & 16.44 \\
\hline
\end{tabular}

Tabla 4.2: Modelo de 4 nodos. Parámetros de referencia, $X_{R}$.

\begin{tabular}{|c|c|c|}
\hline $\begin{array}{c}\text { Nomenclatura } \\
\text { modelo }\end{array}$ & $\begin{array}{c}\text { Correspondencia } \\
\text { parámetro }\end{array}$ & $\begin{array}{c}\text { Valor } \\
{\left[\mathrm{W} \mathrm{K}^{-1}\right]}\end{array}$ \\
\hline$G_{L 12}$ & $X_{1}$ & 0.11 \\
$G_{L 13}$ & $X_{2}$ & 0.12 \\
$G_{L 14}$ & $X_{3}$ & 0.13 \\
$G_{L 23}$ & $X_{4}$ & 0.14 \\
$G_{L 24}$ & $X_{5}$ & 0.15 \\
$G_{L 34}$ & $X_{6}$ & 0.16 \\
\hline
\end{tabular}

Para analizar el modelo se van a considerar 3 casos diferentes. En el primer caso se consideran los seis parámetros para el ajuste, por lo que el sistema de ecuaciones (4.1) será un sistema indeterminado, en el segundo caso se fijan tres parámetros de manera que el sistema sea sobredeterminado, y finalmente se estudia el comportamiento de modelo utilizando 3 parámetros de ajuste y 3 casos de carga.

\subsection{Caso indeterminado}

El caso indeterminado aparece cuando hay más parámetros que temperaturas de ajuste $\left(N_{P}>N_{N}\right)$. El modelo tiene de esta manera varios conjuntos de valores de los parámetros que ajustan el modelo de referencia con un error $E_{T}=0{ }^{\circ} \mathrm{C}$.

Esta situación no tiene sentido físico, ya que existe más de un conjunto de parámetros que representa la realidad. Aún así es interesante ya que el modelo reproducirá las temperaturas de referencia.

Para ilustrar este caso supongase que se pretende ajustar los 6 parámetros que aparecen en la Tabla 4.2, usando como referencia, es decir, como objetivo, las tem- 
peraturas de la Tabla 4.1. Como punto de partida se emplean los parámetros que aparecen en la Tabla 4.3, que han sido seleccionados de manera aleatoria.

Tabla 4.3: Modelo de 4 nodos. Parámetros iniciales en el caso indeterminado, $X_{0}$.

\begin{tabular}{|c|c|}
\hline Parámetro & Valor inicial $\left[\mathrm{W} \mathrm{K}^{-1}\right]$ \\
\hline$X_{1}$ & 0.08 \\
$X_{2}$ & 1.13 \\
$X_{3}$ & 0.07 \\
$X_{4}$ & 0.11 \\
$X_{5}$ & 1.10 \\
$X_{6}$ & 1.20 \\
\hline
\end{tabular}

En la Figura 4-2 se muestran la evolución del RSS, $R_{T}$ en 5 iteraciones. Después de dos iteraciones el error $E_{T}$ pasa de $0.7^{\circ} \mathrm{C}$ a $1.3 \times 10^{-3}{ }^{\circ} \mathrm{C}$. En la Tabla 4.4 se muestra las temperaturas de los nodos del modelo a lo largo de las 5 iteraciones. En la columna $T_{R}$ se muestra la temperatura de referencia y la iteración 0 representa las temperaturas iniciales (obtenidas a partir de los parámetros iniciales, dados en la Tabla 4.3).

Se puede observar que el algoritmo converge rápidamente y el modelo proporciona unas temperaturas iguales a las temperaturas de referencia. Pero esto no significa que los parámetros encontrados sean los correctos. En la Tabla 4.5 se muestran los valores encontrados y se aprecia que estos valores difieren de los parámetros de referencia, $X_{R}$, si bien este conjunto de parámetros proporciona las temperaturas de referencia. Este caso puede resultar de gran interés en modelos matemáticos reducidos en donde hay que preservar la arquitectura del modelo aunque las conductancias térmicas no sean necesariamente las reales.

Tabla 4.4: Modelo de 4 nodos. Evolución de temperaturas del modelo en el caso indeterminado.

\begin{tabular}{|c|c|c|c|c|c|c|c|}
\cline { 3 - 8 } \multicolumn{2}{c|}{} & \multicolumn{7}{|c|}{ Iteraciones } \\
\hline Node & $T_{R}\left[{ }^{\circ} \mathrm{C}\right]$ & $0\left[{ }^{\circ} \mathrm{C}\right]$ & $1\left[{ }^{\circ} \mathrm{C}\right]$ & $2\left[{ }^{\circ} \mathrm{C}\right]$ & $3\left[{ }^{\circ} \mathrm{C}\right]$ & $4\left[{ }^{\circ} \mathrm{C}\right]$ & $5\left[{ }^{\circ} \mathrm{C}\right]$ \\
\hline 1 & 8.755 & 8.471 & 8.568 & 8.748 & 8.755 & 8.755 & 8.755 \\
2 & 15.617 & 15.738 & 15.655 & 15.609 & 15.617 & 15.617 & 15.617 \\
3 & 19.146 & 19.147 & 19.233 & 19.166 & 19.146 & 19.146 & 19.146 \\
4 & 16.437 & 17.090 & 16.817 & 16.441 & 16.437 & 16.437 & 16.437 \\
\hline
\end{tabular}




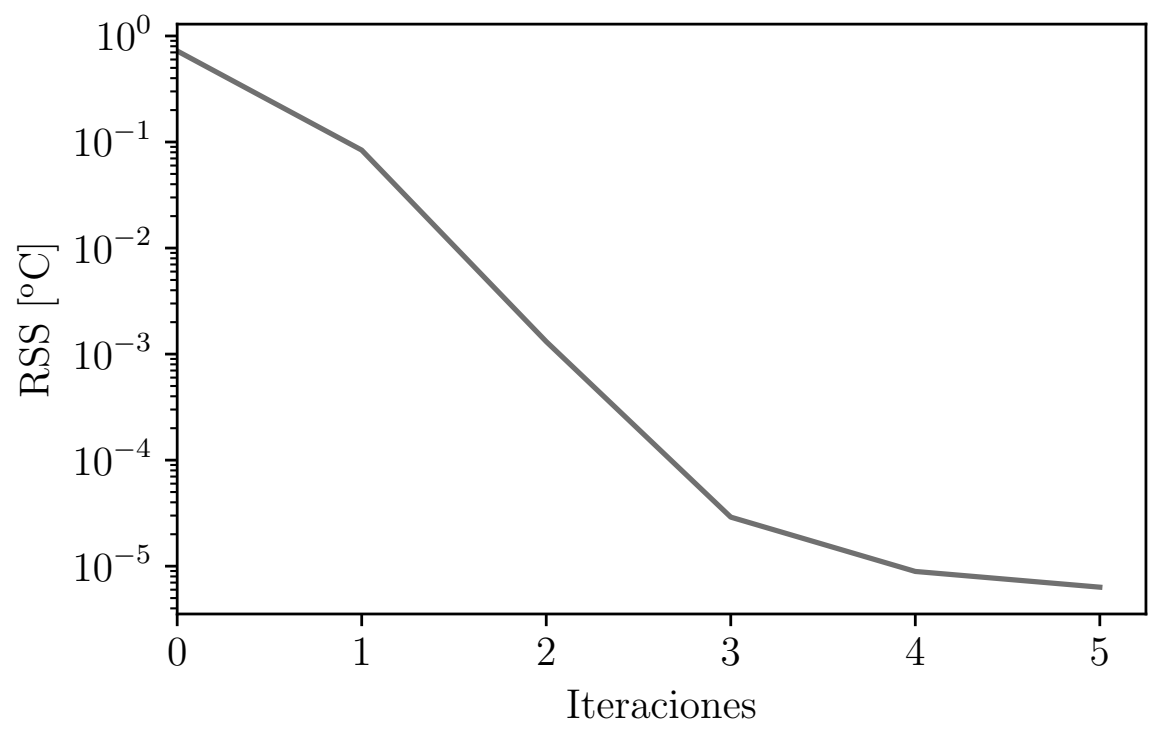

Figura 4-2: Modelo de 4 nodos. Caso indeterminado. Evolución de la RSS.

Tabla 4.5: Modelo de 4 nodos. Parámetros encontrados en el caso indeterminado.

\begin{tabular}{|c|c|}
\hline Parámetro & Valor encontrado $\left[\mathrm{W} \mathrm{K}^{-1}\right]$ \\
\hline$X_{1}$ & 0.118 \\
$X_{2}$ & 0.105 \\
$X_{3}$ & 0.143 \\
$X_{4}$ & 0.154 \\
$X_{5}$ & 0.160 \\
$X_{6}$ & 0.201 \\
\hline
\end{tabular}

Hay que tener en cuenta, que el modelo ha sido ajustado unicamente en un caso de carga. Por lo tanto es muy probable que no sea útil en cualquier otro caso diferente.

Es fácil poner en evidencia que con este modelo y disponiendo de los 6 parámetros de ajuste se pueden encontrar varios conjuntos de valores de los parámetros que proporcionan las mismas temperaturas (dentro de un error determinado). Para ello es suficiente comenzar la búsqueda de los valores de parámetros desde un punto diferente al mostrado en la Tabla 4.3. Los nuevos valores están mostrados en la Tabla 4.6 .

Con estos nuevos valores iniciales y después de 5 iteraciones se recuperan las temperaturas de referencia pero con un conjunto completamente diferente de parámetros de modelo. La evolución de las temperaturas se muestra en la Tabla 4.7 mientras que 
Tabla 4.6: Modelo de 4 nodos. Nuevos valores iniciales en el caso indeterminado, $X_{0}$.

\begin{tabular}{|c|c|}
\hline Parámetro & Valor inicial $\left[\mathrm{W} \mathrm{K}^{-1}\right]$ \\
\hline$X_{1}$ & 0.12 \\
$X_{2}$ & 0.09 \\
$X_{3}$ & 0.10 \\
$X_{4}$ & 0.08 \\
$X_{5}$ & 0.13 \\
$X_{6}$ & 0.16 \\
\hline
\end{tabular}

los parámetros encontrados en la Tabla 4.8 .

Tabla 4.7: Modelo de 4 nodos. Evolución temperaturas del modelo en el caso indeterminado.

\begin{tabular}{|c|c|c|c|c|c|c|c|}
\cline { 3 - 8 } \multicolumn{2}{c|}{} & \multicolumn{7}{|c|}{ Iteraciones } \\
\hline Node & $T_{R}\left[{ }^{\circ} \mathrm{C}\right]$ & $0\left[{ }^{\circ} \mathrm{C}\right]$ & $1\left[{ }^{\circ} \mathrm{C}\right]$ & $2\left[{ }^{\circ} \mathrm{C}\right]$ & $3\left[{ }^{\circ} \mathrm{C}\right]$ & $4\left[{ }^{\circ} \mathrm{C}\right]$ & $5\left[{ }^{\circ} \mathrm{C}\right]$ \\
\hline 1 & 8.755 & 8.522 & 8.442 & 8.638 & 8.768 & 8.752 & 8.755 \\
2 & 15.617 & 15.343 & 15.462 & 15.395 & 15.467 & 15.619 & 15.617 \\
3 & 19.146 & 19.764 & 19.674 & 19.201 & 19.082 & 19.150 & 19.146 \\
4 & 16.437 & 16.709 & 16.901 & 16.918 & 16.614 & 16.439 & 16.437 \\
\hline
\end{tabular}

Tabla 4.8: Modelo de 4 nodos. Parámetros encontrados en el caso indeterminado.

\begin{tabular}{|c|c|}
\hline Parámetro & Valor encontrado $\left[\mathrm{W} \mathrm{K}^{-1}\right]$ \\
\hline$X_{1}$ & 0.069 \\
$X_{2}$ & 0.156 \\
$X_{3}$ & 0.118 \\
$X_{4}$ & 0.095 \\
$X_{5}$ & 0.007 \\
$X_{6}$ & 0.082 \\
\hline
\end{tabular}

La gráfica 4-3 muestra la evolución del RSS.

En este caso el RSS después de 5 iteraciones es de $\sim 10^{-3}{ }^{\circ} \mathrm{C}$, más alto que en el caso anterior. Observando la primera iteración incluso aumenta el RSS, lo que habría sido motivo de parada del algoritmo del apartado 3.2.3 si no se hubiera fijado el número total de iteraciones como criterio. Este comportamiento se puede arreglar ajustando el valor de la tasa máxima de variación de los parámetros, $c_{v}$, pero no se va a cambiar ya que el objetivo es comparar entre los diferentes casos en igualdad de condiciones. 


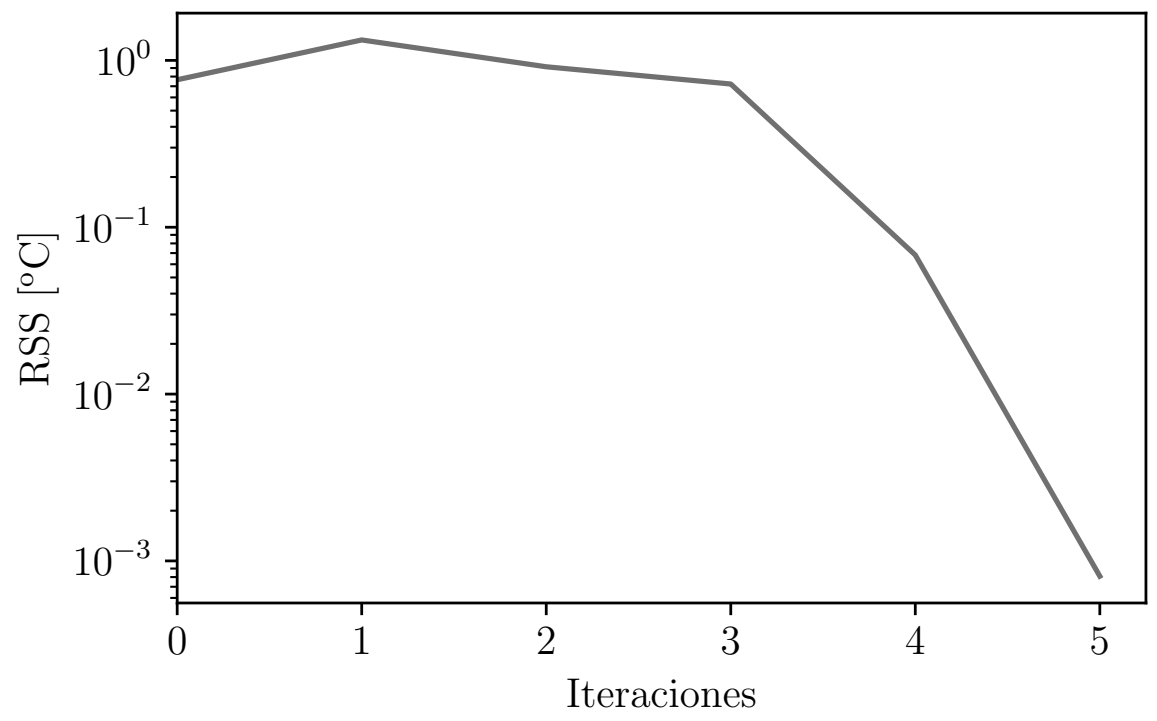

Figura 4-3: Modelo de 4 nodos indeterminado. Evolución RSS.

\subsection{Caso sobredeterminado}

En el caso sobredeterminado, hay más temperaturas de referencia que parámetros ajustables. Este es el caso de mayor interés en la actualización de modelos matemáticos ya que es el caso que se da con más frecuencia. Hay que tener en cuenta que habitualmente el número de parámetros que se usan para el ajuste es menor al número de temperaturas que se pretende utilizar.

Al tener un sistema sobredeterminado, se busca el conjunto de parámetros con el que mejor se ajustan los resultados del modelo a las temperaturas de referencia. Se va a utilizar el modelo de 4 nodos descrito en la Figura 4-1, pero en esta ocasión manteniendo fijos los parámetros $X_{4}, X_{5}$ y $X_{6}$. Dispondremos, por lo tanto de tres parámetros de ajuste.

Para comenzar, hay que inicializar los valores de parámetros del modelo empleando los que aparecen en la Tabla 4.9, donde las 3 últimas filas corresponden a los parámetros que no se van a ajustar (han sido fijados con el valor de $X_{R}$ mostrado en la Tabla 4.2, por lo tanto se espera encontrar una solución que sea igual a $X_{R}$ para el resto de parámetros.

En primer lugar se comprueba la influencia de cada parámetro. En la Figura 4-4 
Tabla 4.9: Modelo de 4 nodos. Parámetros iniciales en el caso sobredeterminado.

\begin{tabular}{|c|c|}
\hline Parámetro & Valor inicial $\left[\mathrm{W} \mathrm{K}^{-1}\right]$ \\
\hline$X_{1}$ & 0.34 \\
$X_{2}$ & 0.25 \\
$X_{3}$ & 0.14 \\
\hline$X_{4}$ & 0.14 \\
$X_{5}$ & 0.15 \\
$X_{6}$ & 0.16 \\
\hline
\end{tabular}

se muestra la variación en la temperatura producida por una variación de un $10 \%$ de cada parámetro. Se observa que el parámetro $X_{1}$ afecta a los nodos 1 y 2 en mayor medida, el parámetro $X_{2}$ a los nodos 1 y 3 y el parámetro $X_{3}$ afecta a los nodos 1 y 4. Utilizando los parámetros iniciales se obtienen las temperaturas $T_{0}$ mostradas en

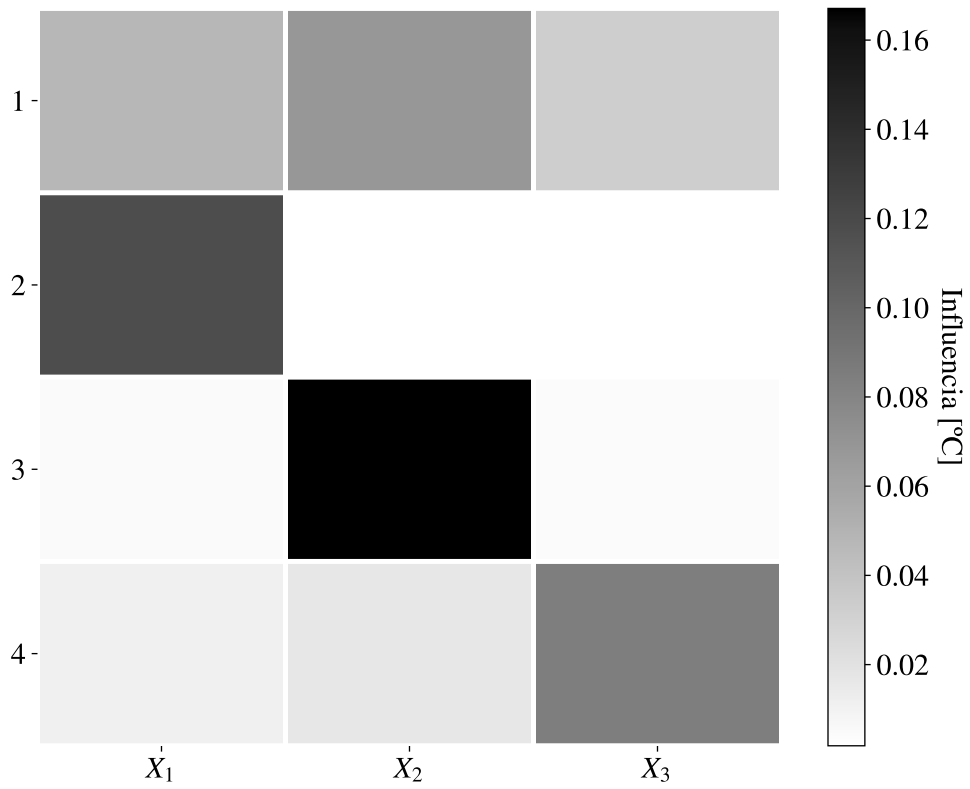

Figura 4-4: Modelo de 4 nodos sobredeterminado.

la Tabla 4.10. Con estos parámetros el error es $\mathrm{RSS}=2.03{ }^{\circ} \mathrm{C}$. Empezando en estas condiciones iniciales, se busca el conjunto de parámetros que ajustan el modelo de manera que se obtengan las temperaturas de referencia (ver Tabla 4.1). Para ello se buscan en el espacio determinado por los tres parámetros $X_{1}, X_{2}$ y $X_{3}$. 
Tabla 4.10: Modelo de 4 nodos sobredeterminado. Temperaturas iniciales.

\begin{tabular}{|c|c|}
\hline Nodo & $T_{i}^{0}\left[{ }^{\circ} \mathrm{C}\right]$ \\
\hline 1 & 9.77 \\
2 & 14.38 \\
3 & 17.94 \\
4 & 16.10 \\
\hline
\end{tabular}

Tabla 4.11: Modelo de 4 nodos. Evolución de las temperaturas obtenidas en con modelo en el caso sobredeterminado.

\begin{tabular}{|c|c|c|c|c|c|c|c|}
\cline { 3 - 8 } \multicolumn{1}{c|}{} & \multicolumn{7}{|c|}{ Iteraciones } \\
\hline Nodo & $T_{R}\left[{ }^{\circ} \mathrm{C}\right]$ & $0\left[{ }^{\circ} \mathrm{C}\right]$ & $1\left[{ }^{\circ} \mathrm{C}\right]$ & $2\left[{ }^{\circ} \mathrm{C}\right]$ & $3\left[{ }^{\circ} \mathrm{C}\right]$ & $4\left[{ }^{\circ} \mathrm{C}\right]$ & $5\left[{ }^{\circ} \mathrm{C}\right]$ \\
\hline 1 & 8.755 & 9.771 & 9.308 & 8.937 & 8.776 & 8.754 & 8.755 \\
2 & 15.617 & 14.375 & 14.822 & 15.239 & 15.568 & 15.618 & 15.617 \\
3 & 19.146 & 17.942 & 18.551 & 19.071 & 19.142 & 19.146 & 19.146 \\
4 & 16.437 & 16.104 & 16.315 & 16.388 & 16.431 & 16.437 & 16.437 \\
\hline
\end{tabular}

Después de 4 iteraciones se llega a la solución deseada. La Tabla 4.11 muestra la evolución de las temperaturas de los cuatro nodos a lo largo de las iteraciones. La evolución del RSS esta representada en la Figura 4-5. Se observa que el modelo converge rápidamente hasta encontrar los parámetros de referencia. Hay que tener en cuenta que en este caso los parámetros $X_{4}, X_{5}$ y $X_{6}$ han sido fijados con su valor de referencia por lo que el error final esperado es cero. Los parámetros encontrados en

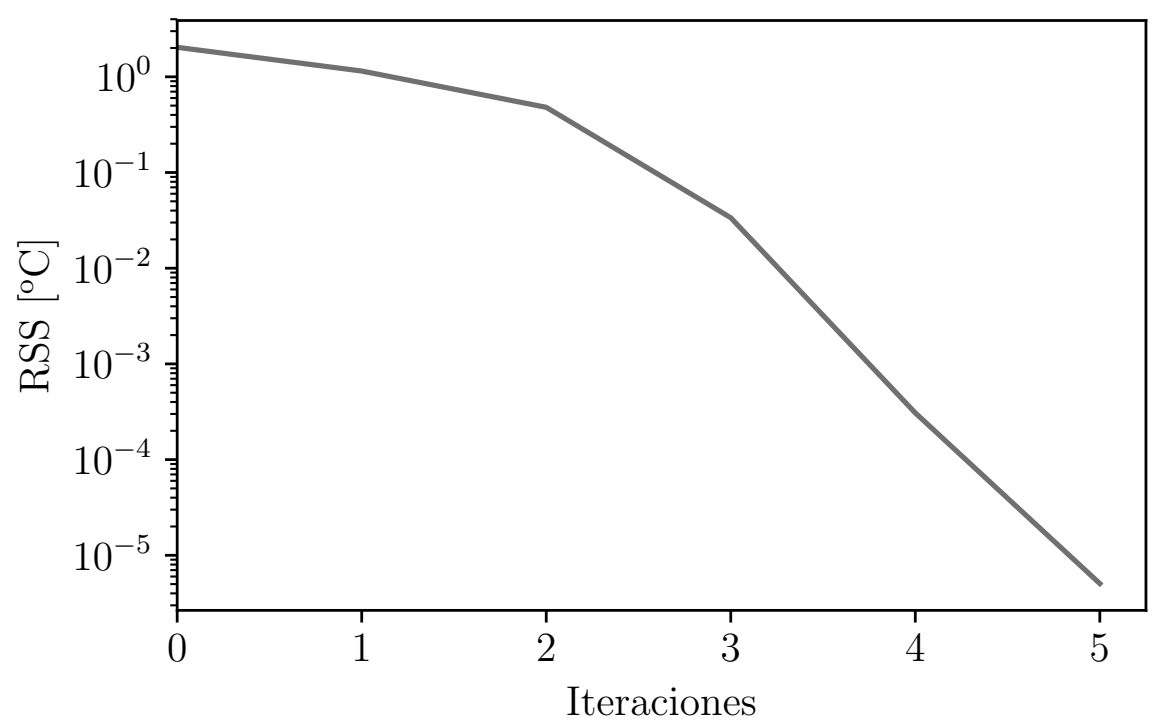

Figura 4-5: Evolución del RSS en el modelo de 4 nodos. Caso sobredeterminado. 
este caso coinciden con los parámetros de referencia. La evolución de estos parámetros está mostrada en la Tabla 4.12. En la cuarta iteración se recuperan los parámetros de referencia (los valores están redondeados a 3 decimales).

Tabla 4.12: Modelo de 4 nodos. Evolución de los valores de los parámetros del modelo en el caso sobredeterminado.

\begin{tabular}{|c|c|c|c|c|c|c|c|}
\cline { 3 - 8 } \multicolumn{2}{c|}{} & \multicolumn{7}{c|}{ Iteraciones } \\
\hline Parámetro & $X_{R}$ & 0 & 1 & 2 & 3 & 4 & 5 \\
\hline$X_{1}\left[\mathrm{~W} \mathrm{~K}^{-1}\right]$ & 0.11 & 0.341 & 0.305 & 0.054 & 0.106 & 0.110 & 0.110 \\
$X_{2}$ [W K $\left.^{-1}\right]$ & 0.12 & 0.252 & 0.080 & 0.120 & 0.120 & 0.120 & 0.120 \\
$X_{3}\left[\mathrm{~W} \mathrm{~K}^{-1}\right]$ & 0.13 & 0.143 & 0.127 & 0.130 & 0.130 & 0.130 & 0.130 \\
\hline
\end{tabular}

\subsubsection{RSS}

Con el fin de tener una visión del comportamiento del algoritmo de ajuste, en las Figuras 4-6, 4-7, 4-8, 4-9 y 4-10 se muestra el comportamiento de RSS en función de los valores de dos parámetros. En este caso se han variado cinco parejas de parámetros, de manera que se puede representar el comportamiento del RSS en función de la distancia de dos parámetros respecto a sus valores de referencia, $\operatorname{RSS}=\operatorname{RSS}\left(x_{j}, x_{k}\right)$.

Sobre cada una de las superficies que se forman se han pintado cuatro trayectorias. Cada una de estas 4 trayectorias corresponde a una combinación de condiciones iniciales, detallada en la Tabla 4.13. Cada iteración está marcada con un punto del color de la trayectoria.

Tabla 4.13: Valores iniciales de las diferentes trayectorias

\begin{tabular}{|c|c|c|}
\hline Trayectoria & $X_{j}^{0}$ & $X_{k}^{0}$ \\
\hline azul & $2 \cdot X_{j}$ & $2 \cdot X_{k}$ \\
naranja & 0.0 & $2 \cdot X_{k}$ \\
verde & 0.0 & 0.0 \\
rojo & $2 \cdot X_{j}$ & 0.0 \\
\hline
\end{tabular}




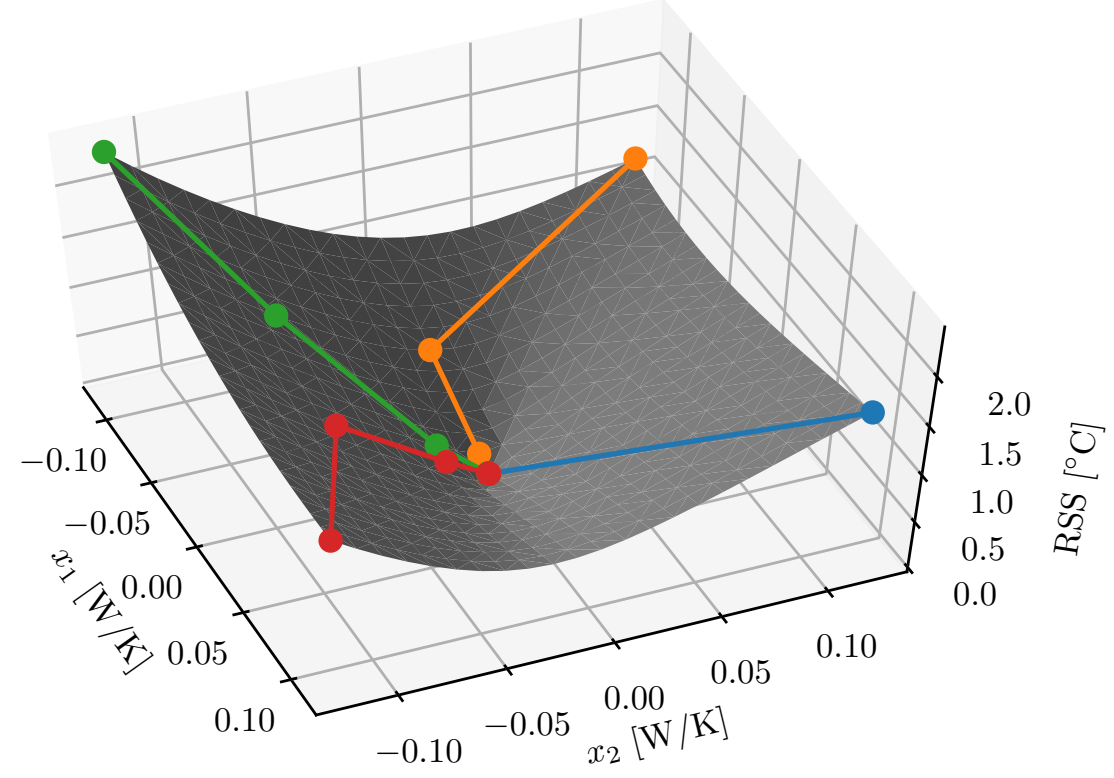

Figura 4-6: RSS. Parámetros $X_{1}$ y $X_{2}$.

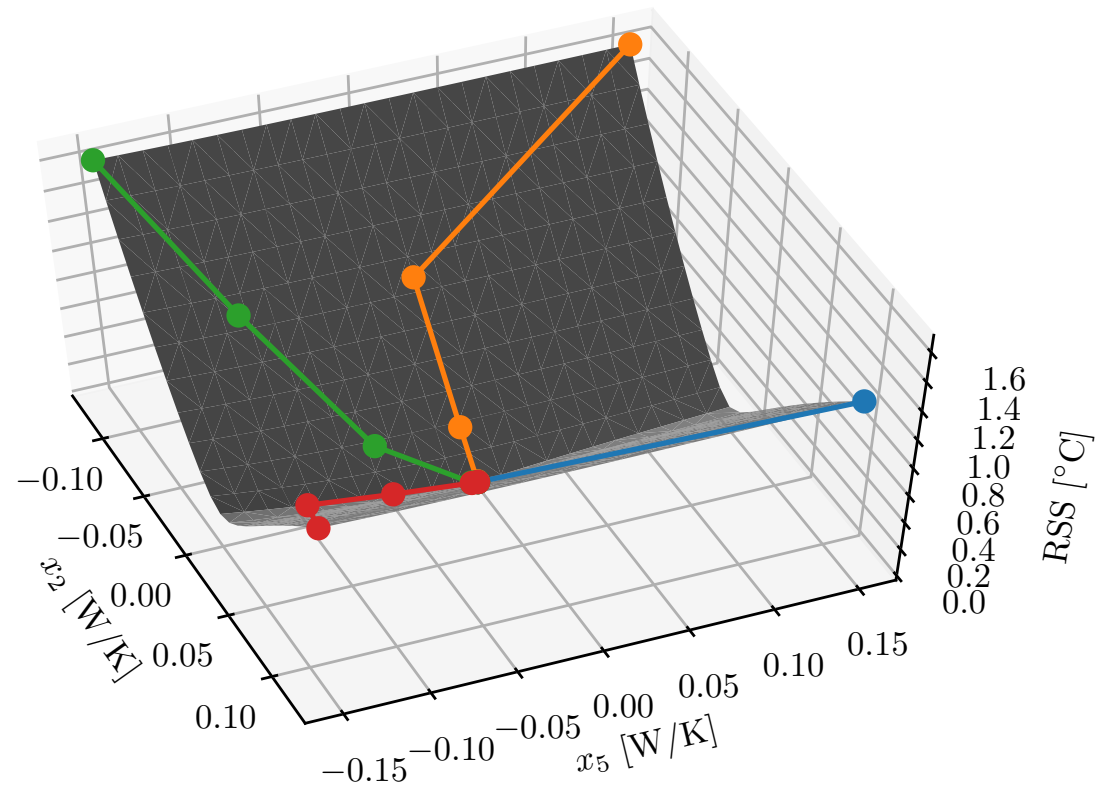

Figura 4-7: RSS. Parámetros $X_{2}$ y $X_{5}$. 


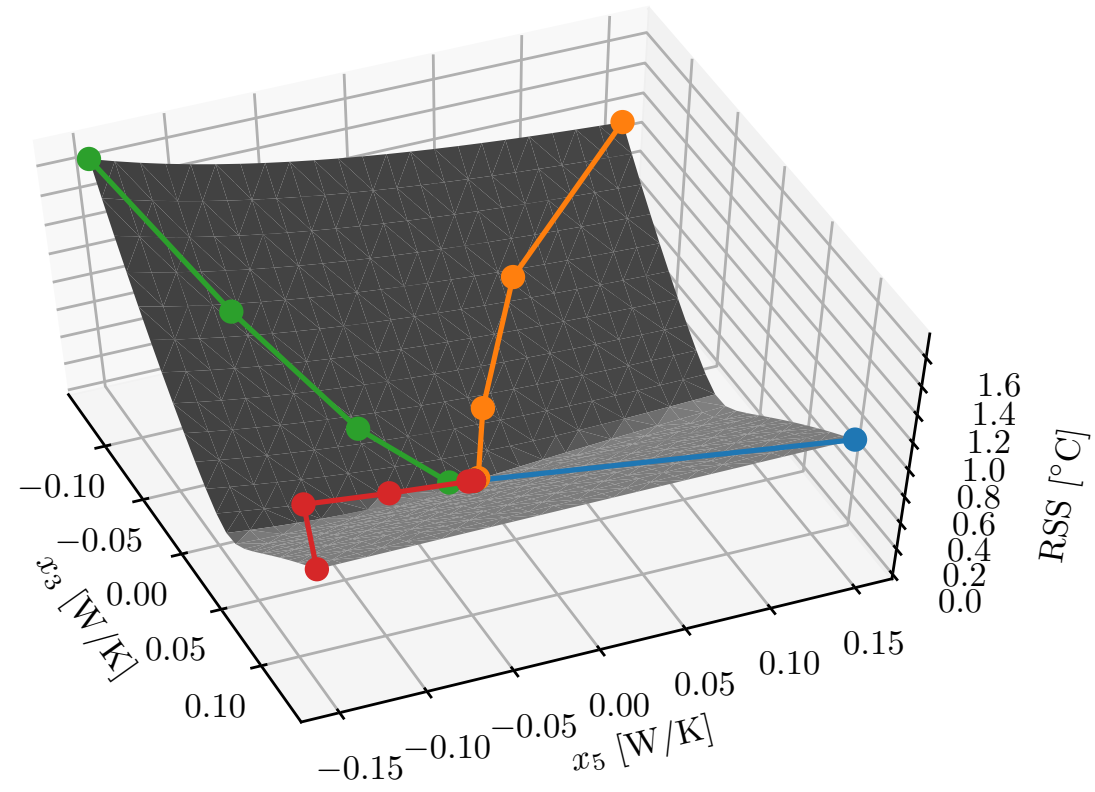

Figura 4-8: RSS. Parámetros $X_{3}$ y $X_{5}$.

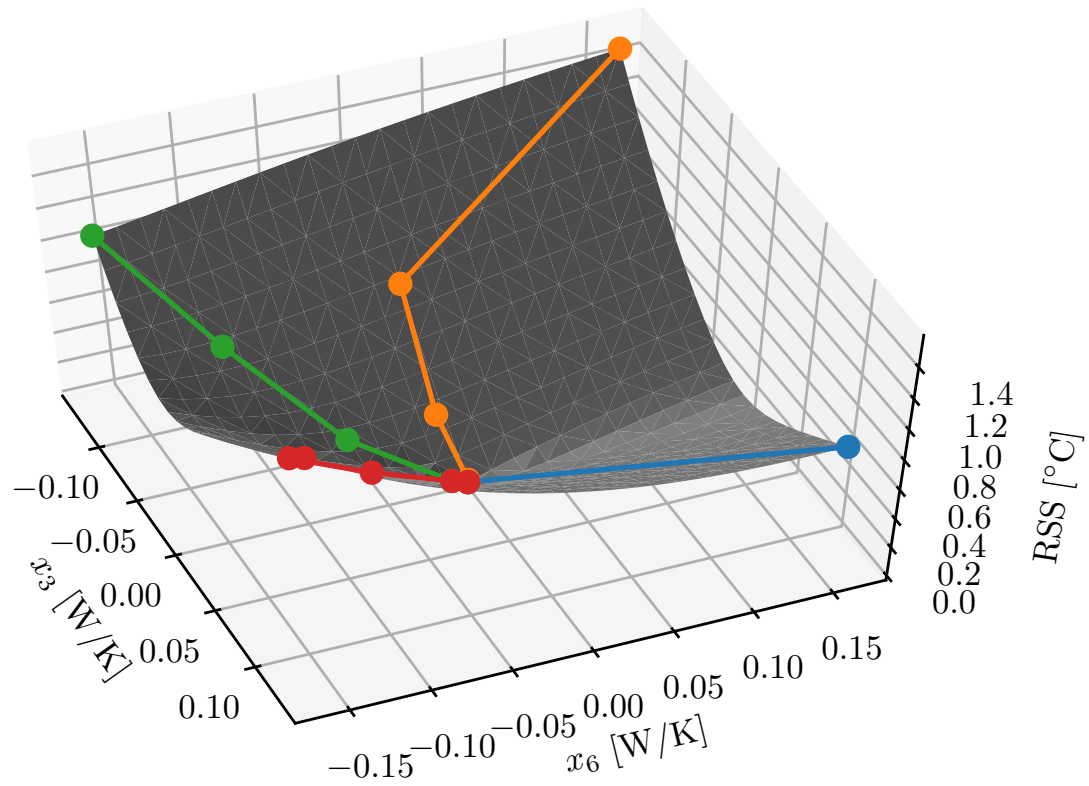

Figura 4-9: RSS. Parámetros $X_{3}$ y $X_{6}$. 


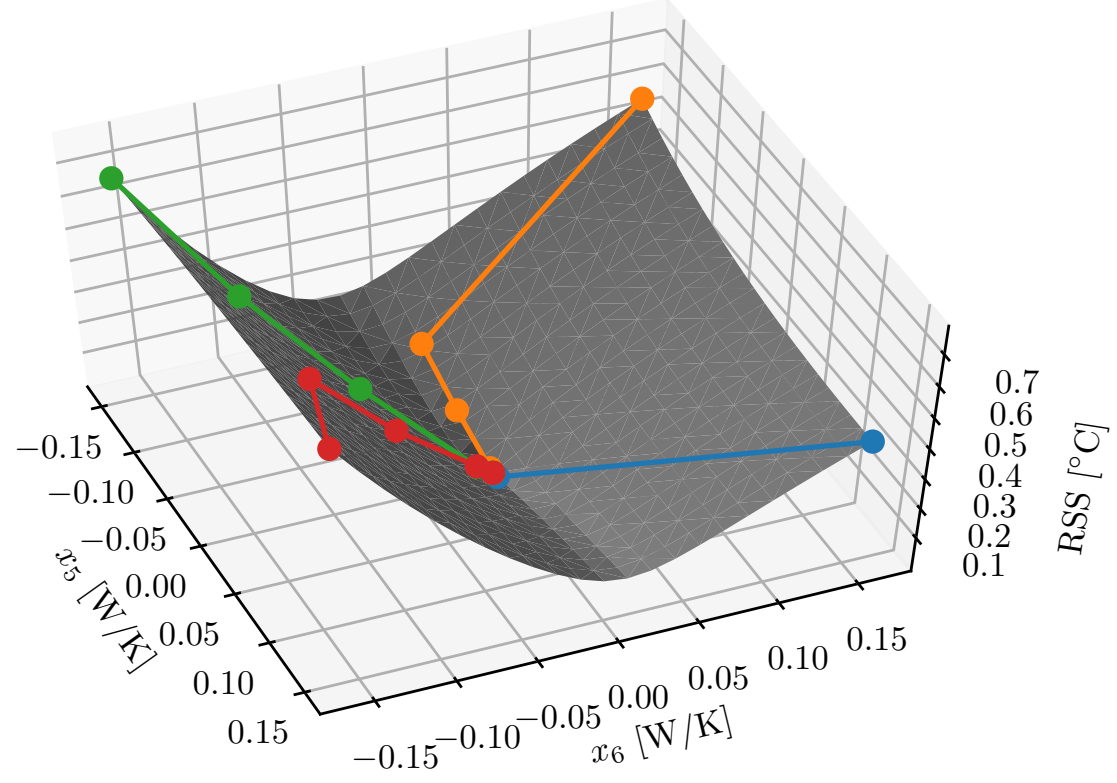

Figura 4-10: RSS. Parámetros $X_{5}$ y $X_{6}$. 
Como regla general se observa que la trayectoria azul es la más rápida a la hora de encontrar la solución. Esto se debe al valor de las condiciones iniciales. Como se ha explicado en el apartado 3.2.3, al empezar a iterar con un valor más alto, la variación máxima permitida, $c_{v}$, es suficiente para corregir el parámetro. Para el resto de trayectorias se observa que con 5 iteraciones llegamos a RSS del orden $10^{-2}{ }^{\circ} \mathrm{C}$.

Se observa también una relación entre el número de iteraciones y el RSS inicial. Para comprobar esta relación se han variado 3 parámetros del modelo al azar y se ha registrado el número de iteraciones necesarias para obtener un RSS menor a $10^{-2}{ }^{\circ} \mathrm{C}$. El resultado se muestra en la Figura 4-11.

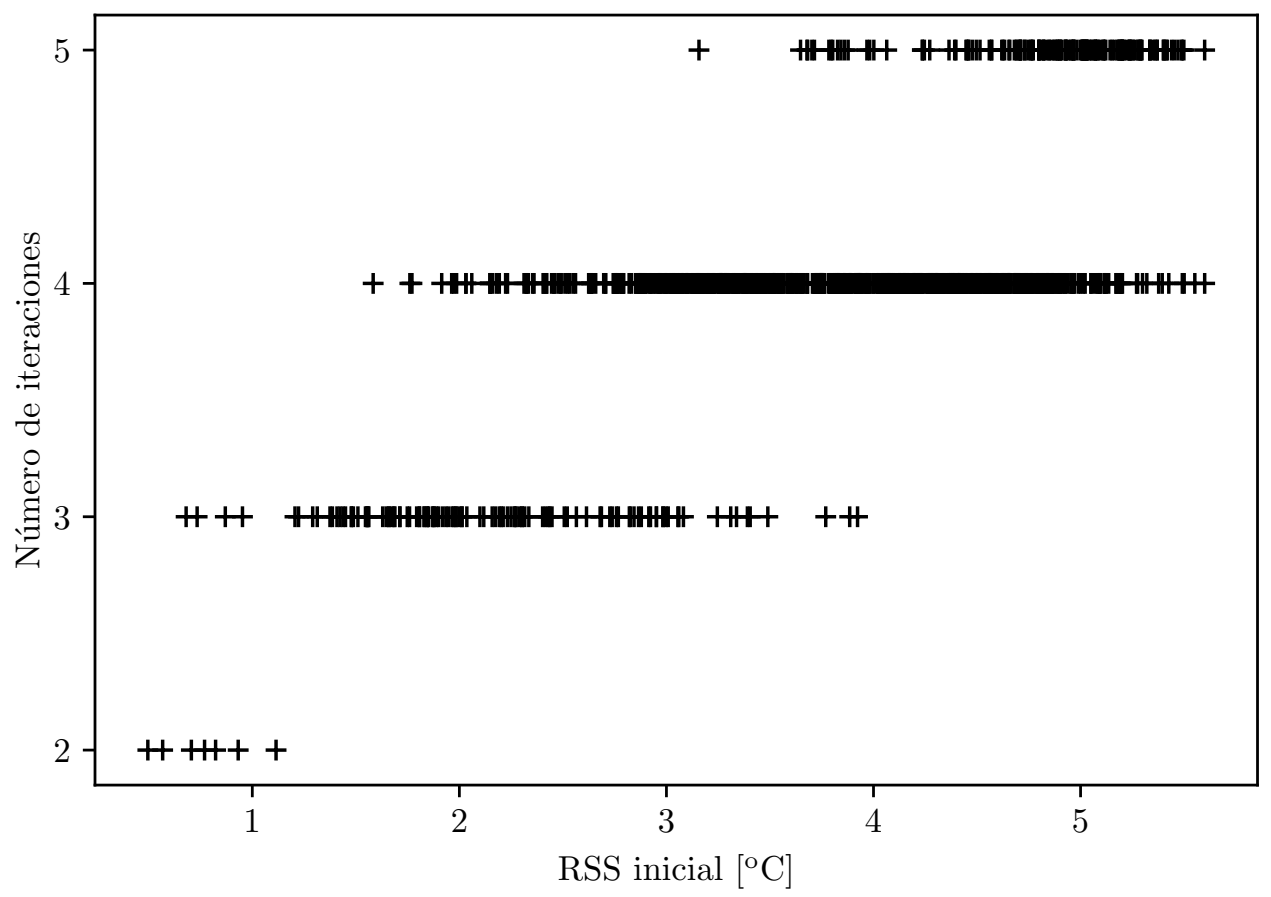

Figura 4-11: RSS. Relación entre el RSS inicial y el número de iteraciones necesario para obtener un $\mathrm{RSS}<10^{-2}{ }^{\circ} \mathrm{C}$.

Se observa el comportamiento esperado, cuanto más alejadas estén las condiciones iniciales de las temperaturas de referencia, más iteraciones se necesitan. 


\subsubsection{Actualización con 3 casos de carga}

Hasta el momento se ha tenido en cuenta un solo caso de carga para el ajuste del modelo, pero como se ha visto en la sección 2.5 el método se puede extender a varios casos de carga, que pueden representar distintos modos de funcionamiento o distintos escenarios. En este apartado se van a considerar tres casos de carga para obtener las correcciones en los parámetros de acuerdo a (2.46). Con el fin de comprobar que el modelo se ajusta correctamente, se utilizan los parámetros de referencia ya presentados en la Tabla 4.2. Para generar los diferentes casos de carga se va a cambiar la disipación interna de cada nodo, como aparece en la Tabla 4.14.

Tabla 4.14: Modelo de 4 nodos. Casos de carga.

\begin{tabular}{|c|c|c|c|}
\cline { 2 - 4 } \multicolumn{1}{c|}{} & \multicolumn{3}{c|}{ Potencia disipada [W] } \\
\hline Nodo & Caso 1 & Caso 2 & Caso 3 \\
\hline 1 & 10.0 & 4.5 & 4.5 \\
2 & 8.0 & 6.0 & 12.0 \\
3 & 12.0 & 4.0 & 4.0 \\
4 & 9.0 & 5.0 & 10.0 \\
\hline
\end{tabular}

Las temperaturas de referencia se obtienen utilizando los parámetros de referencia en los diferentes casos de carga. En la Tabla 4.15 se muestra la temperatura de referencia de estos 3 casos.

Tabla 4.15: Modelo de 4 nodos. Temperaturas de referencia.

\begin{tabular}{|c|c|c|c|}
\cline { 2 - 4 } \multicolumn{1}{c|}{} & \multicolumn{3}{|c|}{ Temperaturas } \\
de referencia $\left[{ }^{\circ} \mathrm{C}\right]$
\end{tabular}

Con el fin de definir unas condiciones de inicio apropiadas hay que variar los valores de los parámetros de referencia. Se van a utilizar tres parámetros de ajuste, $X_{1}, X_{2}$ y $X_{3}$. Los tres parámetros restantes del modelo se mantienen fijos (con el valor de referencia mostrado en la Tabla 4.2). De estas manera se asegura que podría encontrarse un error próximo a cero. 
Tabla 4.16: Modelo de 4 nodos. Temperaturas iniciales de los tres casos de carga.

\begin{tabular}{|c|c|c|c|}
\cline { 2 - 4 } \multicolumn{1}{c|}{} & \multicolumn{3}{|c|}{$\begin{array}{c}\text { Temperaturas } \\
\text { iniciales }\left[{ }^{\circ} \mathrm{C}\right]\end{array}$} \\
\cline { 2 - 4 } \multicolumn{1}{c|}{} & Caso 1 & Caso 2 & Caso 3 \\
\hline$T_{1}^{0}$ & 9.57 & 4.82 & 6.55 \\
$T_{2}^{0}$ & 13.90 & 8.57 & 14.92 \\
$T_{3}^{0}$ & 18.20 & 7.73 & 10.10 \\
$T_{4}^{0}$ & 16.87 & 9.28 & 16.61 \\
\hline
\end{tabular}

En la Figura 4-12 se muestra la evolución del RSS en cada caso de carga, se observa que en tres iteraciones es menor que $10^{-3}{ }^{\circ} \mathrm{C}$. En la Figura 4-13 se muestra la variación de los parámetros de ajuste con el número de iteraciones. Se observa como con menos de 5 iteraciones se recuperan los parámetros de referencia $X_{1}=0.11 \mathrm{~W} \mathrm{~K}^{-1}, X_{2}=$ $0.12 \mathrm{~W} \mathrm{~K}^{-1}$ у $X_{3}=0.13 \mathrm{~W} \mathrm{~K}^{-1}$.

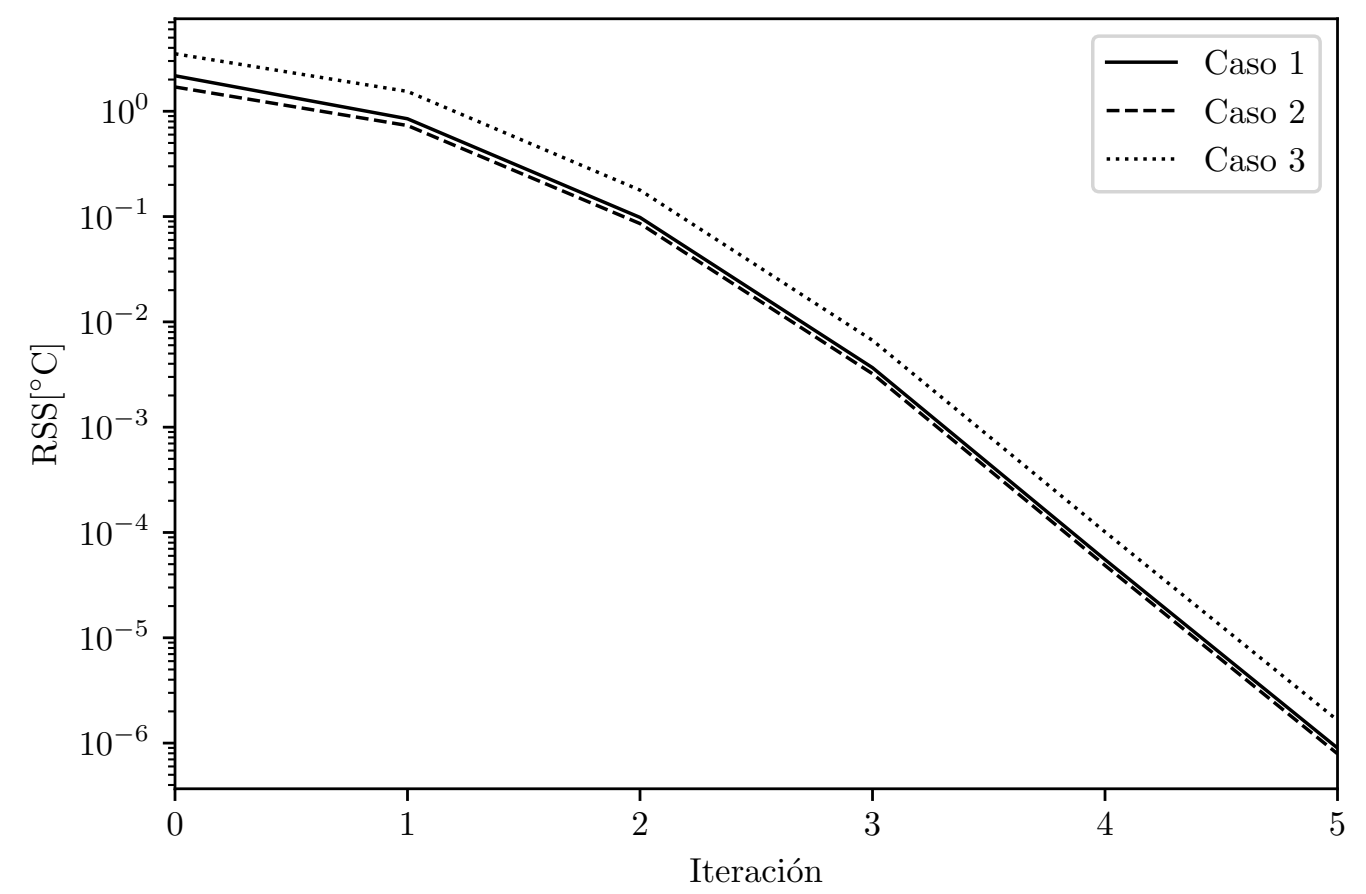

Figura 4-12: Modelo de 4 nodos. Variación del RSS con el número de iteraciones 


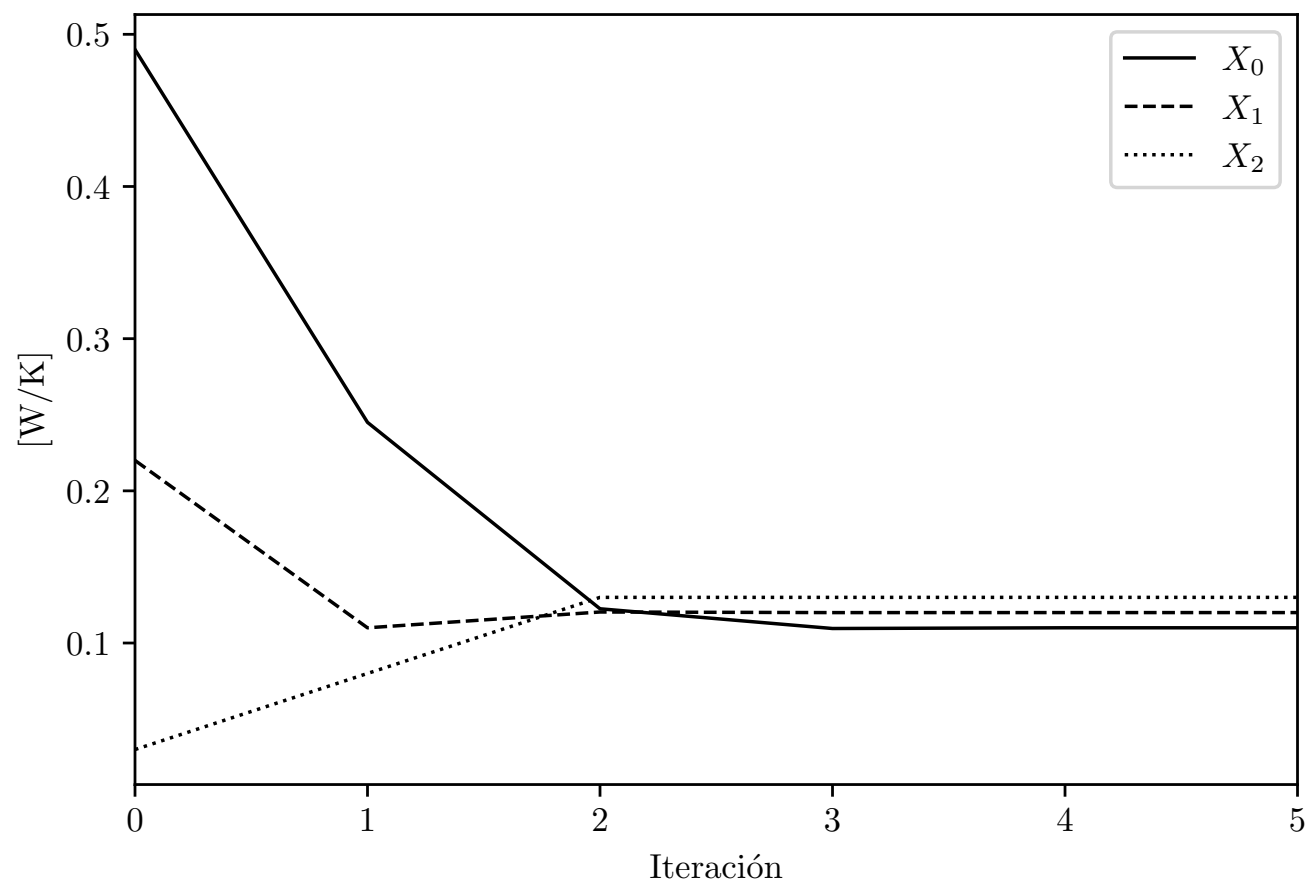

Figura 4-13: Modelo de 4 nodos. Variación del valor de los parámetros de ajuste con el número de iteraciones 


\section{Capítulo 5}

\section{Aplicación a Solar Orbiter PHI O-Unit}

\subsection{Contexto}

El instrumento Polarimetric and Helioseismic Imager (PHI) [59,60] forma parte de la misión científica Solar Orbiter [61] de la Agencia Espacial Europea (ESA). Solar Orbiter es una misión de clase M cuyo lanzamiento está previsto para el año 2020 con un cohete Atlas V desde Cabo Cañaveral. El objetivo principal de la misión consiste en estudiar la heliosfera y los efectos de la actividad solar sobre esta. Para ello cuenta con numerosos instrumentos de los cuales destacan los denominados remote sensing ${ }^{1}$, entre los que se encuentra PHI.

La órbita que seguirá el satélite permitirá observar el sol con latitudes de hasta $30^{\circ}$ y se acercará a una distancia de hasta 0.28 AU. En contraposición, durante el afelio llegará hasta las 1.4 AU. Es por ello que el entorno que se va a encontrar el satélite y los instrumentos embarcados durante la misión va a ser muy hostil, lo que requiere un diseño del control térmico muy exigente.

PHI se compone de una unidad electrónica, PHI E-Unit y de un banco óptico con dos telescopios, PHI O-Unit. El diseño térmico de ambos subsistemas ha sido responsabilidad del Instituto Universitario 'Ignacio da Riva', IDR/UPM [63].

\footnotetext{
${ }^{1}$ Instrumentos de detección remota que no interfieren con el objeto a observar.
} 


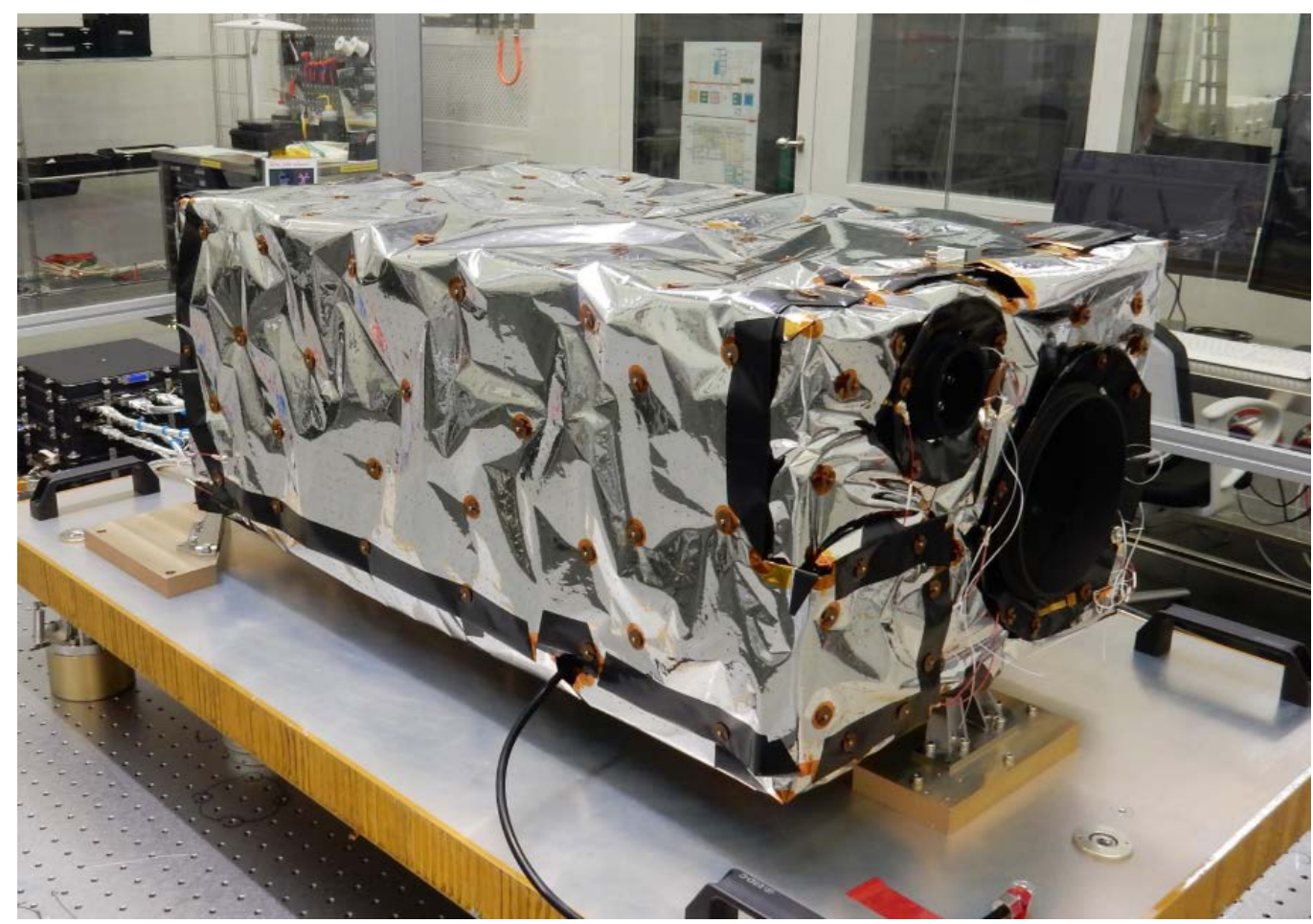

Figura 5-1: SO/PHI O-Unit con las mantas térmicas instaladas [62].

En el diseño de la unidad electrónica [15] también se ha aplicado el método presentado en esta tesis $[64,65]$. El RTMM de PHI E-Unit se ha ajustado respecto a su respectivo DTMM utilizando el método GIPI propuesto. Sin embargo, este modelo contaba únicamente con 7 nodos (ver Figura 1-3) y es por ello que se ha considerado de más interés mostrar el ajuste de PHI O-Unit en vez de este por su mayor complejidad. En las referencias señaladas se puede encontrar la información del ajuste de PHI E-Unit.

El instrumento PHI pertenece al grupo de instrumentos que se denominan internally mounted, lo que significa que es una unidad alojada en el interior del satélite. Por lo tanto su entorno tanto conductivo como radiativo esta controlado por la nave. Todas las cargas térmicas que entren o se disipen en la unidad tienen que ser evacuadas a través de las interfaces térmicas hacia a los radiadores. En el caso de PHI O-Unit estas interfaces son tres Hot Elements (HEs) y un Cold Element (CE). El CE está dedicado a enfriar exclusivamente el sensor del Focal Plane Asssembly (FPA) mientras que los tres HEs mantienen la temperatura general de la unidad. 
En las próximas secciones se presentan tanto el modelo térmico detallado como el reducido y el ajuste realizado sobre este último. Los resultados obtenidos se comentan junto con una comparación con otros métodos y un estudio del efecto del coeficiente de variación máxima, $c_{v}$.

\subsection{Modelo térmico matemático}

\subsubsection{Modelo térmico matemático detallado}

El modelo matemático detallado de la unidad óptica de PHI esta formado por un total del 7497 nodos, de los cuales 7133 tienen representación geométrica. Estos nodos están agrupados siguiendo una estructura similar a los subsistemas que componen la unidad. En la Tabla 5.1 se presentan los diferentes equipos del instrumento junto con el número de nodos que los componen y el identificativo que se utiliza. Este modelo además incluye las cavidades del interior del satélite (S/C Cavities), Feedthroughs y las ventanas (HREWs) debido a la necesidad de modelar un entorno realista para la unidad. Estos modelos, aunque estén incluidos en el modelado no forman parte del GTMM de PHI O-Unit. Se utilizan como condición de contorno y sus temperaturas vienen determinadas por la plataforma y pueden cambiar correspondiendo a las diferentes fases de la misión.

En la Figura 5-2 se muestra el modelo geométrico detallado con parte de la MLI (en amarillo) retirada para que se pueda ver el interior del instrumento.

Para el diseño y análisis de la unidad se han estudiado varios casos tanto transitorios como estacionarios que incluyen modos de operación nominal, desapuntamientos y fases no operativas. Cada caso estudiado tiene una cantidad de cargas térmicas diferente tanto en distribución como en cantidad. En PHI O-Unit estas cargas son principalmente las que llegan del sol (QS) a través de las dos ventanas del instrumento, la radiación infrarroja (IR) procedente del entorno, la disipación electrónica interna y los heaters que controlan algunos de los elementos ópticos, en particular los cristales líquidos (LCVRs) y el Filtergraph. A pesar de que las HREWs actúan 
Tabla 5.1: Identificación y número de nodos del modelo matemático de PHI O-Unit

\begin{tabular}{|l|l|c|}
\hline PHI OPT Part & Id & No $^{\text {de nodos }}$ \\
\hline Connectors Panel & CP & 4 \\
Common Power Converter & CPC & 426 \\
Correlation Tracker & CT & 366 \\
Front Block & FB & 1354 \\
Full Disk Telescope & FDT & 592 \\
Filtergraph & FG & 630 \\
Focal Plane Assembly & FPA & 834 \\
Feed Select Mechanism & FSM & 24 \\
Bottom Sandwich Panel & HC & 340 \\
HRT PMP & HPMP & 32 \\
HRT Re-Focus Mechanism & HRM & 156 \\
HRT Mirror 1 & M1 & 126 \\
HRT Mirror 2 & M2 & 31 \\
Mirror 4 & M4 & 6 \\
Optics Unit Mounting Feet & MF & 60 \\
Optics Unit MLI & MLI & 880 \\
Optical baffles & OB & 58 \\
Rear Block & RB & 1160 \\
Struts & ST & 24 \\
URP & URP & 3 \\
\hline Aperture Collars & AC & 26 \\
Contaminating Collars & CC & 176 \\
Cold Element Interface & CE & 1 \\
\hline
\end{tabular}

de filtro y solo dejan pasar un $3.2 \%$ de la radiación incidente, la radiación solar que entra en la unidad es del orden de $10 \mathrm{~W}$ cuando el satélite se encuentra en el perihelio. Además hay que tener en cuenta la radiación infrarroja proveniente tanto de los feedthroughs como de las HREWs, que supone alrededor de $5 \mathrm{~W}$. La disipación interna de la electrónica y los heaters son del orden de $6 \mathrm{~W}$ y $4 \mathrm{~W}$, respectivamente.

En esta tesis se utilizan únicamente los casos extremos operativos que se corresponde con los casos caliente y el frío, ambos en régimen estacionario. La selección de estos casos es la adecuada para asegurar que durante todo su periodo operativo las temperaturas de la unidad se encuentren dentro de la envolvente determinada por estos casos. 


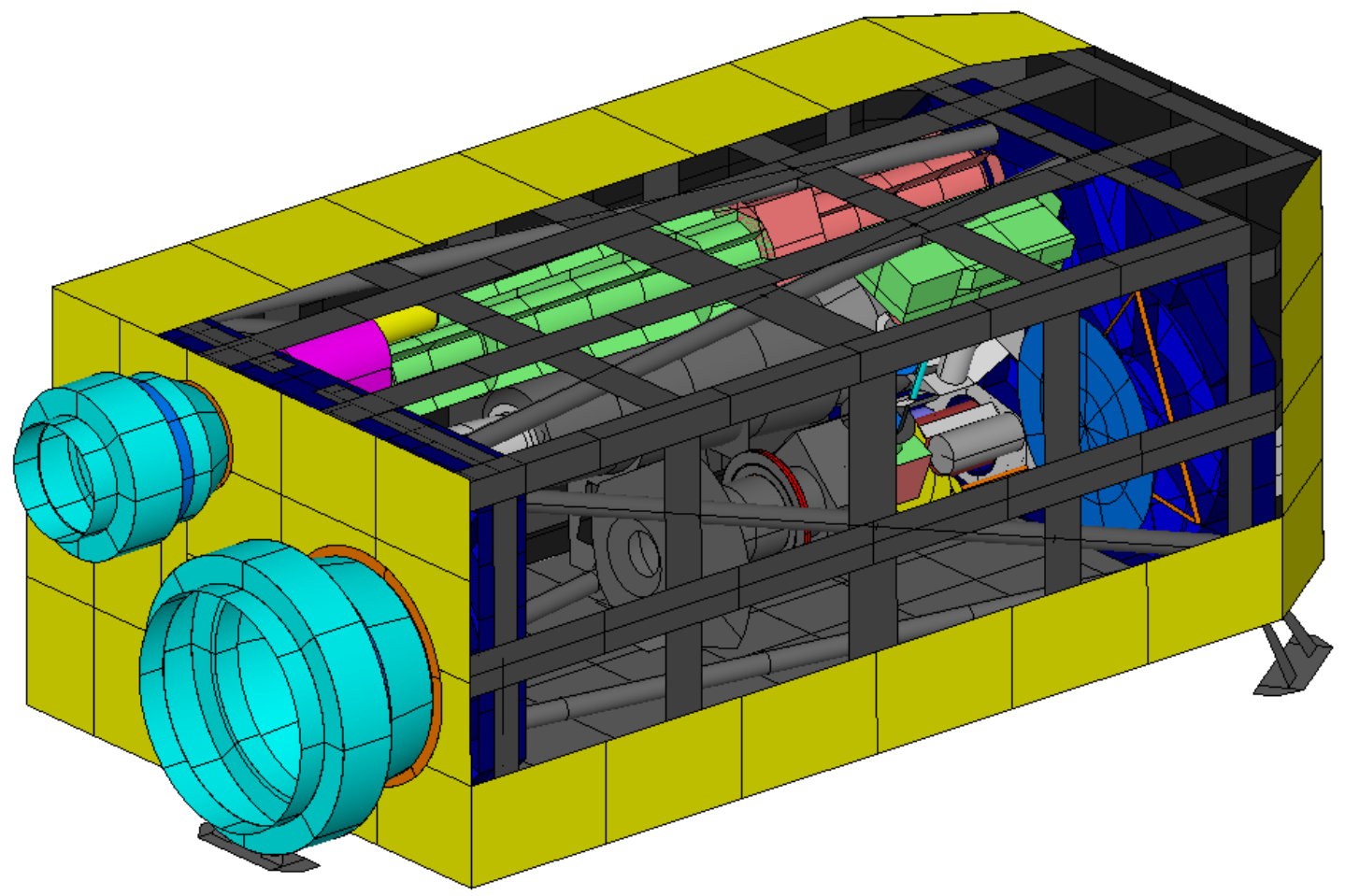

Figura 5-2: Modelo geométrico detallado. SO/PHI O-Unit.

\subsubsection{Modelo térmico matemático reducido}

El modelo geométrico y matemático reducido deriva del modelo detallado. Se ha elaborado a partir de éste, teniendo en cuenta las geometrías y resultados obtenidos. En la Figura 5-3 se muestra el modelo reducido con parte de la MLI apartada para que se pueda observar el interior. Durante el proceso de reducción hay que seguir una serie de normas que varían dependiendo de la misión. En este caso han sido:

- Restricción en el número de nodos. Un límite de 50 nodos, que teniendo en cuenta los 7 utilizados por la unidad electrónica, quedan 43 disponibles para PHI O-Unit.

- Representación de los nodos. Todos los nodos presentes en el DTMM deben de estar presentes en alguno de los nodos en el RTMM. Esto implica que en numerosos casos un nodo del modelo reducido representa a varias partes de subsistemas del modelo detallado.

- Casos ajustados. El modelo reducido se tiene que comportar igual que el deta- 
llado en al menos dos casos operativos.

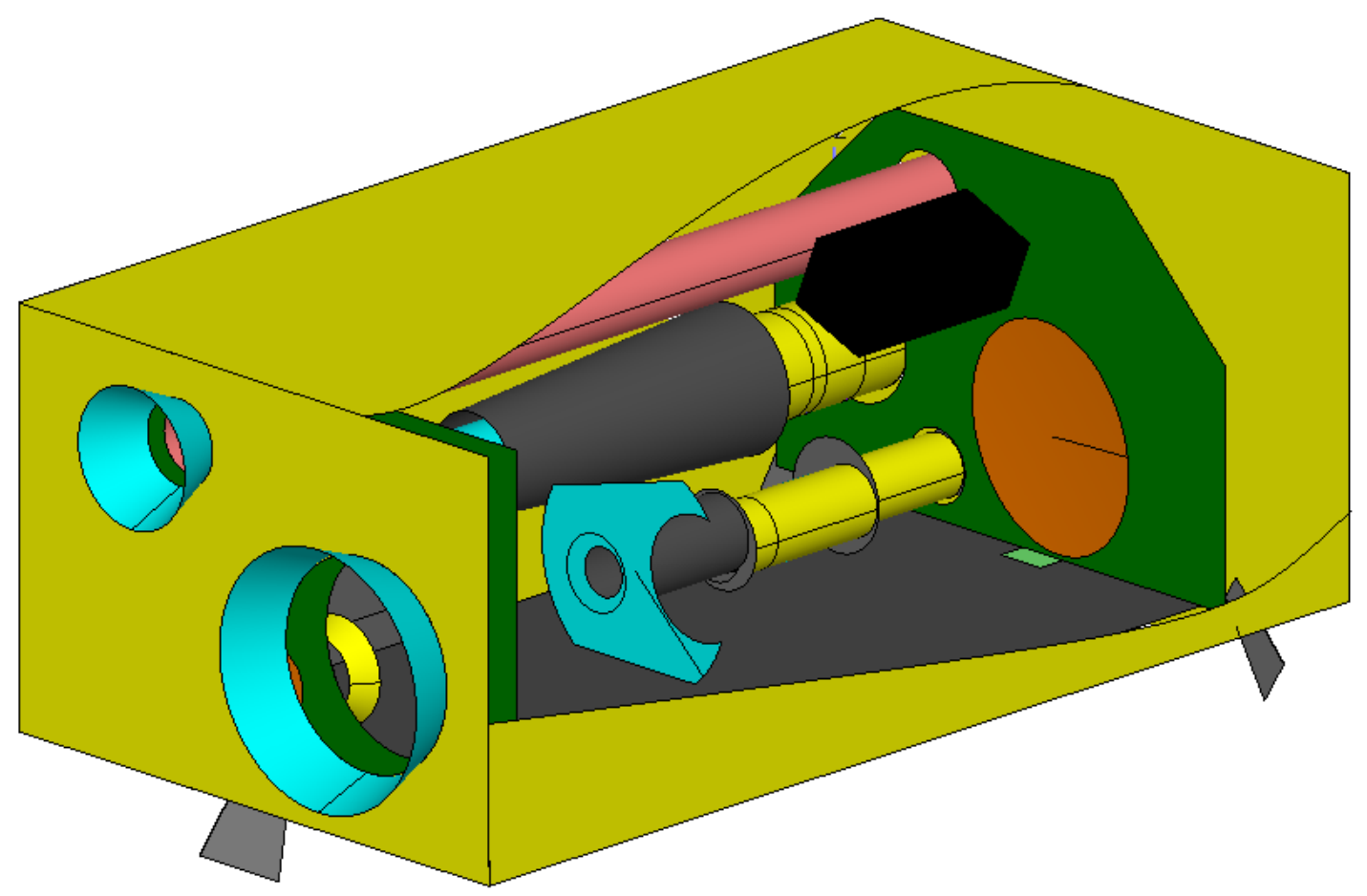

Figura 5-3: Modelo geométrico reducido. SO/PHI O-Unit.

El modelo reducido se ha creado siguiendo una aproximación tradicional, al contrario de los métodos semi-automáticos que se han descrito en la sección 1. El primer paso ha sido identificar las partes del modelo, que por su interés, tienen que tener una representación en el reducido.Es el caso del sensor de la FPA y del CT, PMPs y FG, ya que en el caso de estas partes, la temperatura tiene que estar controlada. Luego se añaden los nodos que representan las partes del modelo más relevantes de manera que se intenta que cada subsistema tenga al menos un nodo. Por último se completa con nodos que debido a su temperatura resulta imprescindible que tengan una representación, para poder realizar un ajuste satisfactorio, ya sea debido al elevado gradiente térmico existente o porque actúan de entorno de otros nodos. Por ejemplo, es imprescindible modelar la estructura de FPA o del FG para poder ajustar la temperatura de los componentes internos.

Hay que asegurar que cada nodo del modelo detallado está presente en un nodo en el modelo reducido. Y cada nodo del modelo reducido represente al menos un nodo en 
el modelo detallado. De esta forma se asegura que no se pierde el significado físico de los parámetros durante el proceso de reducción en cuanto a representación se refiere. La lista de nodos resultante se presenta en la Tabla 5.2. Estos nodos están identificados siguiendo la numeración presente en el modelo matemático. El primer nodo es 0 porque se refiere al primer elemento en la matriz. Los nodos a partir del número 35 están separados por una línea porque representan las interfaces conductivas, como se explica más adelante. Como se observa, hay subsistemas que están compuestos por varios nodos, como es el caso del Filtergraph (FG), mientras que otros se han reducido solo a uno, como es el caso del espejo primario M1. Si comparamos la Tabla 5.2 con la Tabla 5.1 podemos ver que solo las partes correspondientes al Connector Panel (CP), y las Struts (ST) no tienen una representación propia en el modelo reducido. Estos grupos se han incluido en otras partes como el Front Block (FB) y el Rear Block $(\mathrm{RB})$.

El modelo geométrico reducido se representa en la Figura 5-4. Este GMM solo se va a utilizar para calcular los $G_{R}$ s. Se ha identificado con etiquetas cada parte. La MLI que envuelve el instrumento se ha suprimido en la imagen para permitir observar el interior.

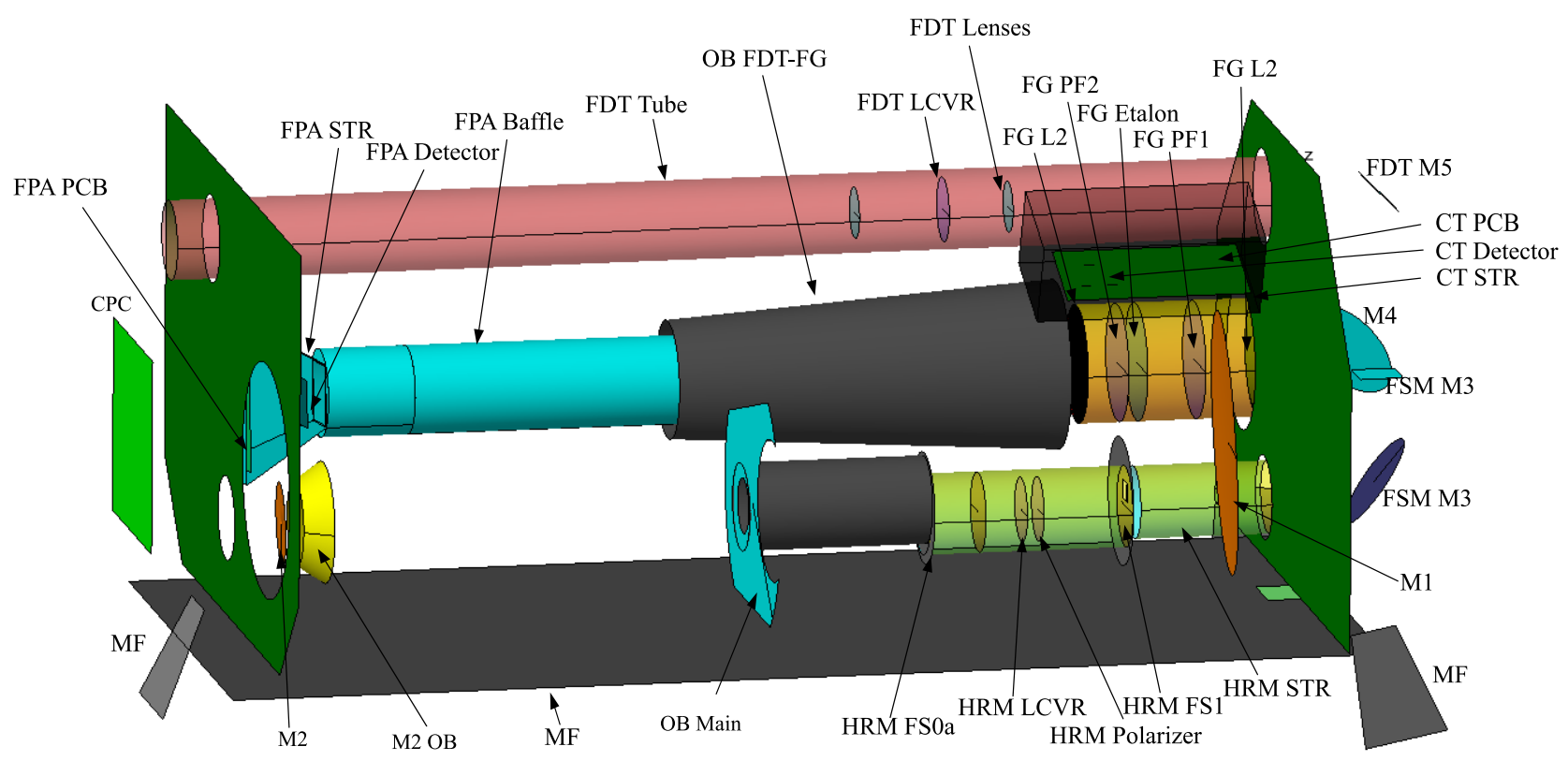

Figura 5-4: PHI O-Unit RGMM (la MLI está retirada). 
El acabado superficial de las caras externas al modelo no se puede modificar y debe coincidir en la medida de lo posible con las equivalentes del modelo detallado. En el caso de PHI O-Unit esta condición es fácil de cumplir puesto que la parte externa del instrumento es fundamentalmente MLI. Hay que tener en cuenta que el modelo reducido se usará en muchos casos para integrarlo dentro de otro modelo matemático, y los responsables de esté volverán a calcular los $G_{R}$ s entre estos modelos. Por este motivo estás superficies tienen que tener el acabado superficial que se espera en la realidad.

No es el caso de las superficies internas del modelo. Por facilidad a la hora de ajustar el modelo se eligen los acabados superficiales más parecidos, pero a la hora de realizar el ajuste fino $\operatorname{los} G_{R}$ s resultantes se pueden variar para conseguir un flujo de calor determinado.

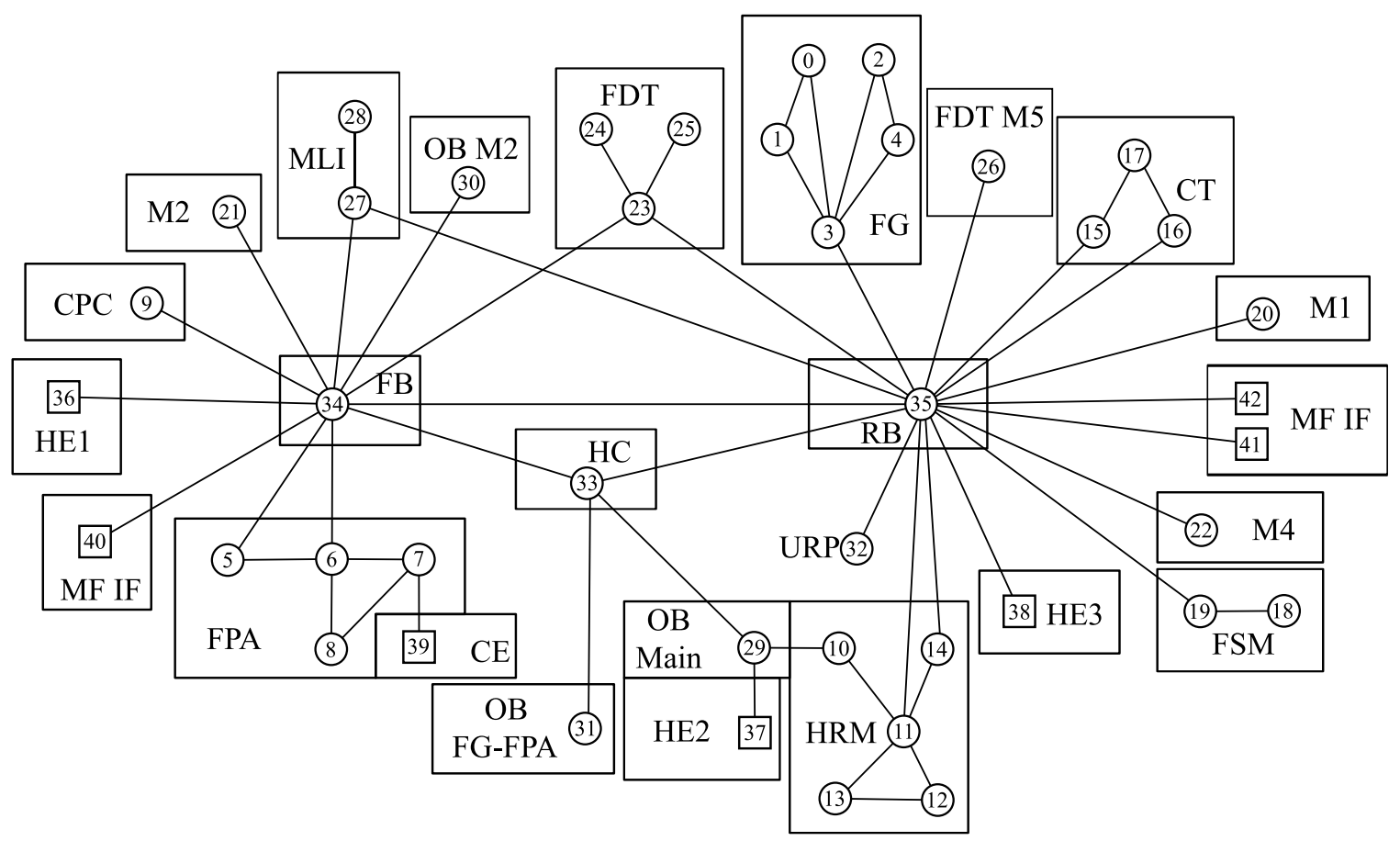

Figura 5-5: Esquema de nodos y $G_{L}$ s. PHI O-Unit.

Una vez el modelo geométrico está creado, hay que introducir toda la información que falta para completar el modelo matemático. Es el caso de las conductancias térmicas lineales, $G_{L} \mathrm{~s}$, entre los nodos. Estás conductancias térmicas representan la medida del intercambio de calor por medio de conducción entre dos nodos, ya sea 
porque representan superficies que están en contacto o pertenecen a la misma pieza. En la Figura 5-5 se muestra el diagrama de nodos con las conductancias lineales $G_{L}$ S, marcadas con una línea.

Estos $G_{L}$ S tienen que representar y representan un camino conductivo real en el modelo. No se pueden añadir caminos nuevos si estos no están presentes en la realidad. De esta manera se preserva el significado físico del modelo matemático. Este hecho no es trivial, ya que en algunos métodos de reducción se recurre a añadir conductacias virtuales entre nodos para poder reproducir las temperaturas predichas por el modelo detallado, como se ha explicado en la sección 1.2.

Aparte de los $G_{L}$ s y $G_{R}$ s el modelo matemático ha de completarse con las condiciones de contorno. Las podemos clasificar en tres tipos:

- Interfaces conductivas. Son nodos con una temperatura fijada que representan los puntos de contacto del instrumento con el satélite. En este caso son los tres Hot Elements (HE) y el Cold Element (CE) que irán a sus respectivos radiadores, y las tres patas del instrumento, que se apoyarán en la bandeja del satélite. Estas siete interfaces están representadas en el diagrama de la Figura 5-5 mediante un cuadrado, nodos del 36 al 42. No es necesario que estos nodos tengan una representación geométrica, no es un requisito e incluso en ocasiones se obvia por simplicidad. En este caso están representados gráficamente con la intención única de poder visualizar su temperatura.

- Interfaces radiativas. Son nodos con obligada representación geométrica que se fijan a una temperatura dada. Este es el caso de la cavidad dentro del satélite y de los Feedthrough (FTs). Solo tienen intercambio de calor con el modelo por medio de radiación.

- Cargas térmicas en los nodos. Estas cargas pueden tener diferente origen, bien la radiación procedente del sol, el albedo o infrarrojo terrestre o la disipación electrónica. Pueden representar también el calor proporcionado por un heater, como es en el caso de los LCVRs y del FG. 
Tabla 5.2: Nodos considerados en el RTMM.

\begin{tabular}{|c|c|c|}
\hline Número de nodo & Subsistema & Parte \\
\hline 0 & FG & Etalon \\
\hline 1 & FG & Pre Filter \\
\hline 2 & FG & Struct Ext \\
\hline 3 & FG & Struct Int \\
\hline 4 & FG & Lenses \\
\hline 5 & FPA & Baffle \\
\hline 6 & FPA & Structure \\
\hline 7 & FPA & APS \\
\hline 8 & FPA & PCB \\
\hline 9 & $\mathrm{CPC}$ & $\mathrm{CPC}$ \\
\hline 10 & HRM & FS0 \\
\hline 11 & HRM & Structure \\
\hline 12 & HRM & LCVR \\
\hline 13 & HRM & Polarizer \\
\hline 14 & HRM & FS1 \\
\hline 15 & $\mathrm{CT}$ & Structure \\
\hline 16 & $\mathrm{CT}$ & Detector \\
\hline 17 & $\mathrm{CT}$ & PCB \\
\hline 18 & FSM & M3 \\
\hline 19 & FSM & FS2 \\
\hline 20 & M1 & M1 \\
\hline 21 & M2 & M2 \\
\hline 22 & M4 & M4 \\
\hline 23 & FDT & Structure Tube \\
\hline 24 & FDT & LCVR \\
\hline 25 & FDT & Lenses \\
\hline 26 & FDT & M5 \\
\hline 27 & MLI & Internal Layer \\
\hline 28 & MLI & External Layer \\
\hline 29 & OB & Main \\
\hline 30 & OB & M2 \\
\hline 31 & OB & FPA-FG \\
\hline 32 & URP & URP \\
\hline 33 & $\mathrm{HC}$ & $\mathrm{HC}$ \\
\hline 34 & FB & FB \\
\hline 35 & $\mathrm{RB}$ & $\mathrm{RB}$ \\
\hline 36 & $\mathrm{HE}$ & HE1 \\
\hline 37 & $\mathrm{HE}$ & HE2 \\
\hline 38 & $\mathrm{HE}$ & HE3 \\
\hline 39 & $\mathrm{CE}$ & $\mathrm{CE}$ \\
\hline 40 & MF IF & MF IF1 \\
\hline 41 & MF IF & MF IF3 \\
\hline 42 & MF IF & MF IF2 \\
\hline
\end{tabular}




\subsection{Ajuste del modelo}

Para el ajuste del modelo matemático reducido se han seleccionado dos casos de carga, los dos casos más significativos para la unidad, que son el caso caliente operativo

y el frío operativo en estado estacionario. Estos dos escenarios determinan el intervalo de operación de todo el instrumento siempre que éste se encuentre en condiciones nominales. Los casos de carga se diferencian entre ellos por la temperatura a la que están sus interfaces y las cargas térmicas. Siguiendo la nomenclatura utilizada en el proyecto, definida por el contratista principal Airbus Defence \& Space UK, estos casos de carga se denominan DC2, para el caso caliente operativo, y PC1 para el caso frío operativo.

En la Tabla 5.3 se muestran las cargas térmicas para los dos casos de carga. Estas cargas tienen fundamentalmente tres fuentes de origen ya que algunas representan las disipaciones electrónicas internas, como pueden ser sensores (FPA APS, CT detector), las tarjetas electrónicas o los motores. Otras son también disipación eléctrica, pero vienen directamente de los heaters, como es el caso del FG Etalon y los LCVRs. En la Tabla 5.3 también aparecen las cargas solares que inciden en el instrumento. Para ello se ha utilizado el RGMM, cambiando ligeramente la geometría de manera que la distribución de las cargas solares dentro del instrumento sea lo más parecida posible a la obtenida con software óptico especializado. Aún así hay parte de la carga incidente, que debido a las limitaciones del modelo reducido, inciden en zonas del interior del instrumento que no deberían.

Debe hacerse notar que la carga infrarroja procedente del entorno, cavidad y Feedthroughs, no se introduce en el modelo directamente como una carga térmica, sino que es parte del resultado obtenido, al considerar como dato las temperaturas de dicho entorno.

Quedan por completar los dos casos de carga definiendo la temperatura del entorno, tanto radiativo como conductivo. En la Tabla 5.4 se recogen éstas temperaturas. Debido a que el instrumento se encuentra dentro del satélite, hay que fijar la temperatura de esta cavidad. Además también son necesarias las temperaturas de las 
Tabla 5.3: Casos de carga. Cargas térmicas.

\begin{tabular}{|c|l|c|c|}
\hline Node & Element & DC2 $[\mathrm{W}]$ & PC1 $[\mathrm{W}]$ \\
\hline 7 & FPA APS & 0.36 & 0.36 \\
8 & FPA PCB & 1.35 & 1.35 \\
16 & CT Detector & 0.07 & 0.07 \\
17 & CT PCB & 1.08 & 1.08 \\
9 & CPC & 0.81 & 0.81 \\
19 & FSM FS2 & 0.20 & 0.20 \\
35 & RB & 0.20 & 0.20 \\
\hline \multicolumn{2}{|c|}{ Total Electrónica } & 4.27 & 4.27 \\
\hline 12 & HRM LCVR & 0.12 & 1.20 \\
24 & FDT LCVR & 0.87 & 0.30 \\
0 & FG Etalon (Oven) & 0.42 & 2.10 \\
\hline \multicolumn{2}{|r|}{ Total heaters } & 1.41 & 3.60 \\
\hline 29 & OB Main & 4.23 & 0.16 \\
30 & OB M2 & 0.45 & 0.00 \\
10 & HRM FS0 & 1.63 & 0.20 \\
14 & HRM FS1 & 0.29 & 0.05 \\
13 & HRM Polarizer & 0.37 & 0.11 \\
19 & FSM FS2 & 0.06 & 0.05 \\
20 & M1 & 0.10 & 0.01 \\
21 & M2 & 0.07 & 0.01 \\
- & Resto del instrumento & 2.47 & 0.22 \\
\hline \multicolumn{2}{|r|}{ Total Solar } & 9.67 & 0.80 \\
\hline
\end{tabular}

interfaces conductivas, que son las más relevantes en cuanto a requisitos. Faltan por indicar las temperaturas de los Feedthroughs, que se han omitido por brevedad.

Tabla 5.4: Casos de carga. Temperatura de las interfaces

\begin{tabular}{|l|c|c|}
\hline Element & $\mathrm{DC} 2\left[{ }^{\circ} \mathrm{C}\right]$ & $\mathrm{PC} 1\left[{ }^{\circ} \mathrm{C}\right]$ \\
\hline Cavidad S/C & 50 & -20 \\
$\mathrm{MF}$ IF & 50 & -20 \\
$\mathrm{HEs}$ & 50 & -20 \\
$\mathrm{CE}$ & -10 & -25 \\
\hline
\end{tabular}

De cara a aplicar el método descrito en esta tesis, GIPI, las temperaturas de referencia vienen definidas a partir de los resultados del modelo matemático detallado, como la media ponderada de la temperatura de cada nodo con su capacidad térmica. De esta manera se espera que durante un análisis transitorio las temperaturas evolucionen de la forma más parecida posible entre los dos modelos. 
En la Tabla 5.5 se muestran las temperaturas de referencia. Se puede ver cómo el FG Etalon mantiene la temperatura en los dos casos. Esto es debido a que es un elemento controlado por medio de heaters. Sucede los mismo con los LCVRs (HRM y FDT), con la única diferencia que en estos su temperatura de consigna varía según el caso.

Antes de proceder con el método de actualización conviene aclarar lo que se denomina como un modelo matemático ajustado. Los criterios de lo que se considera un modelo ajustado no están contemplados en ninguna norma y suelen variar de un proyecto a otro. Bajo el marco de las misiones ESA, los proyectos siguen lo recomendado en $[4,66]$. Estos criterios vienen del ajuste de los modelos matemáticos respecto a unas temperaturas de referencia determinadas en campañas de ensayo. De la misma manera se recomienda aplicar los mismos criterios para el ajuste de un RTMM contra un DTMM. Estos baremos son:

- Diferencia en las temperaturas del modelo detallado respecto de la media ponderada del reducido:

- Equipos y elementos controlados:

- Media $<2 \mathrm{~K}$.

○ Individualmente $<3 \mathrm{~K}$.

- Superficies externas sin MLI $<3 \mathrm{~K}$.

- Elementos estructurales $<3 \mathrm{~K}$.

- $\mathrm{MLI}<15 \mathrm{~K}$.

- Desviación estándar de la temperatura de todos los nodos $<3 \mathrm{~K}, 1 \sigma$.

- Diferencia de potencia disipada o calor transmitido a través de las interfaces del equipo:

- Por conducción $<5 \%$.

- Por radiación $<5 \%$.

- Disipación $<10 \%$.

Si bien estos límites se dan únicamente como ejemplo, son utilizados sin apenas cambios en la mayoría de los proyectos. En el proyecto de Solar Orbiter se hace una consideración aparte para el calor a través de las interfaces siendo aceptable una 
Tabla 5.5: Temperaturas de referencia.

\begin{tabular}{|c|l|c|c|}
\hline Nodo & Etiqueta & DC2 $\left[{ }^{\circ} \mathrm{C}\right]$ & $\mathrm{PC} 1\left[{ }^{\circ} \mathrm{C}\right]$ \\
\hline 0 & FG Etalon & 65.9 & 66.5 \\
1 & FG Pre Filter & 65.2 & 63.3 \\
2 & FG Struct Ext & 57.0 & 14.2 \\
3 & FG Struct Int & 65.9 & 65.7 \\
4 & FG Lenses & 57.4 & 17.5 \\
5 & FPA Baffle & 51.5 & -18.3 \\
6 & FPA Structure & 46.8 & -16.9 \\
7 & FPA APS & 2.1 & -20.7 \\
8 & FPA PCB & 53.3 & -9.5 \\
9 & CPC CPC & 53.6 & -16.4 \\
10 & HRM FS0 & 59.7 & -17.6 \\
11 & HRM Structure & 56.4 & -11.5 \\
12 & HRM LCVR & 75.0 & 45.2 \\
13 & HRM Polarizer & 78.5 & 46.5 \\
14 & HRM FS1 & 54.8 & -14.5 \\
15 & CT Structure & 54.1 & -16.3 \\
16 & CT Detector & 55.6 & -14.7 \\
17 & CT PCB & 64.2 & -4.6 \\
18 & FSM M3 & 52.4 & -17.2 \\
19 & FSM FS2 & 54.0 & -15.3 \\
20 & M1 M1 & 52.0 & -18.7 \\
21 & M2 M2 & 51.1 & -19.8 \\
22 & M4 M4 & 52.7 & -13.6 \\
23 & FDT Structure Tube & 54.9 & -17.6 \\
24 & FDT LCVR & 74.8 & -5.4 \\
25 & FDT Lenses & 54.2 & -18.0 \\
26 & FDT M5 & 51.9 & -18.5 \\
27 & MLI Internal Layer & 52.9 & -18.5 \\
28 & MLI External Layer & 50.3 & -19.9 \\
29 & OB Main & 54.4 & -19.0 \\
30 & OB M2 & 58.4 & -19.5 \\
31 & OB FPA-FG & 53.7 & -16.3 \\
32 & URP URP & -19.5 \\
33 & HC HC & -18.8 \\
34 & FB FB & -19.7 \\
35 & RB RB & -18.7 \\
\hline
\end{tabular}

diferencia de $0.1 \mathrm{~W}$ si el total es menor a $1 \mathrm{~W}$. 


\subsubsection{Identificación de parámetros}

Como se ha comentado en el apartado 3.1 para poder aplicar el método GIPI los parámetros del modelo han de cumplir una serie de requisitos. En este caso, al tratarse de un modelo reducido, los parámetros del modelo van a coincidir con las conductancias lineales $\left(G_{L} \mathrm{~s}\right)$ y radiativas $\left(G_{R} \mathrm{~s}\right)$.

Este modelo está pensado para ser integrado en el modelo completo del satélite. Durante este proceso los $G_{R}$ s del modelo se vuelven a calcular, de manera que una variación de sus valores dificulta la labor del ingeniero que tiene que estar sobrescribiendo constantemente los datos. Por este motivo y a no ser que sea imprescindible, los $G_{R}$ S no se van a considerar parte de los parámetros del modelo. Nos vamos a centrar en ajustar los $G_{L}$ s únicamente (en la Figura 5-5 están representados por líneas). Vamos a identificar los parámetros como (nodo A, nodo B) ya que representan el camino conductivo que va de un nodo a otro. De esta manera si por ejemplo ajustamos el parámetro $(34,5)$ se puede identificar claramente que estamos ajustando el $G_{L 345}$.

De esta manera, inicialmente hay 53 parámetros, tantos como $G_{L}$ s tiene el modelo. Debido al proceso de reducción utilizado, el valor de muchos de estos parámetros apenas afectan en el comportamiento de las temperaturas del modelo. Por esto, lo primero que hay que hacer es identificar aquellos parámetros que más afecten. Para esto se calcula la matriz de influencia (3.1) que se muestra en la Figura 5-6. En el eje de las ordenadas se muestran los nodos del modelo y en el eje de abscisas los diferentes parámetros del modelo. En este caso se observa que la influencia está fundamentalmente centrada entorno a la diagonal principal. Esto se debe a que debido a las características del modelo, los nodos que se encuentran juntos físicamente aparte de estar numerados consecutivamente tienen $G_{L} \mathrm{~S}$ (parámetros) entre ellos, y se han ordenado convenientemente.

La matriz de influencia permite además comprobar gráficamente el cumplimiento de los requisitos definidos en el apartado 3, ya que se puede determinar la observabilidad de tanto los resultados como de los parámetros.

Para poder cuantificar la influencia que tiene un parámetro de manera global, 


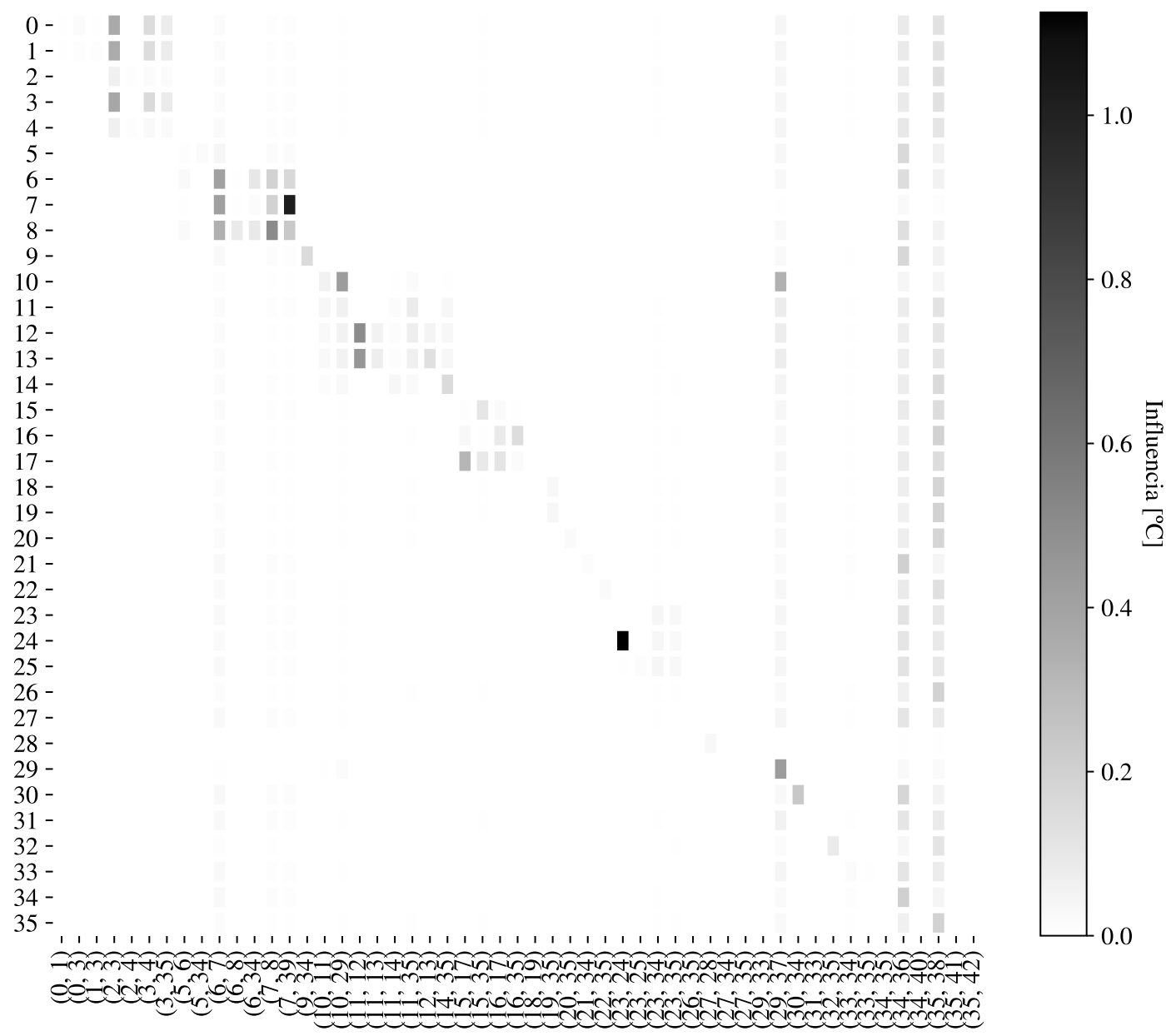

Figura 5-6: PHI OPT. Influencia parámetros.

conviene definir la influencia global como la norma de los vectores columna de $\mathbf{I}_{\mathbf{X}} \mathbf{y}$ se normaliza con la máxima de estas normas:

$$
I_{X_{j}}=\frac{\left[\sum_{i=1}^{N_{N}} I_{X i j}^{2}\right]^{1 / 2}}{\max _{j}\left(\left[\sum_{i=1}^{N_{N}} I_{X i j}^{2}\right]^{1 / 2}\right)}
$$

donde $I_{X i j}$ son los elementos de la matriz de influencia $\mathbf{I}_{\mathbf{X}}$. De esta manera podemos analizar el impacto que tiene cada parámetro en el modelo y seleccionar los más adecuados para el ajuste. En la Figura 5-7 se muestra la influencia global de cada 
parámetro.

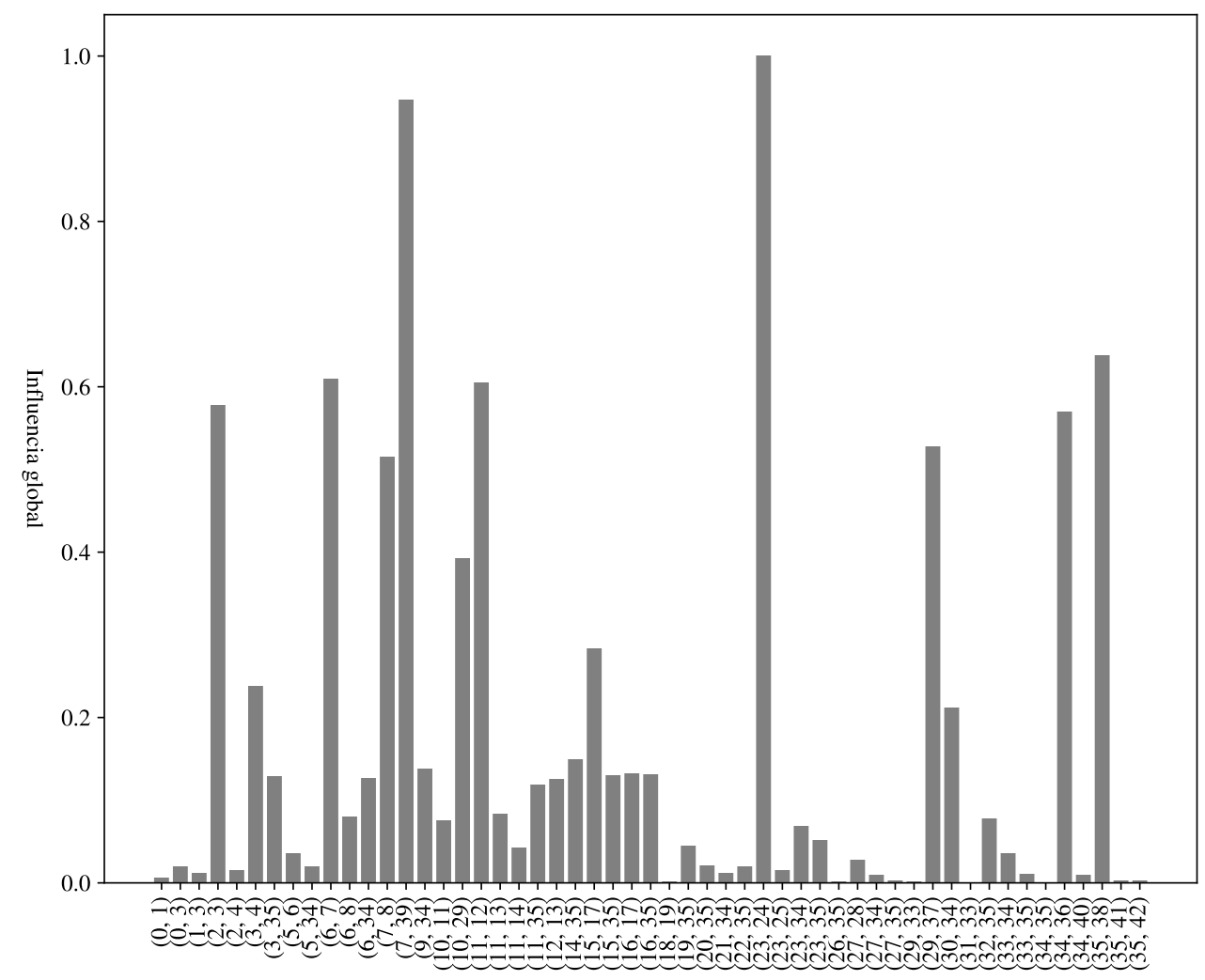

Figura 5-7: PHI O-Unit. Influencia global $I_{X}$ de los parámetros de modelo.

Se observa que hay parámetros que no influyen o influyen muy poco en las temperaturas del modelo, como es el caso de los $G_{L} \mathrm{~S}(18,19),(26,35),(27,35),(31,33)$, $(34,35),(35,41)$ y $(35,42)$. Para representar gráficamente de una manera clara qué elementos físicos se corresponden con estos parámetros, en la Figura 5-8 se ha superpuesto una escala de color que va desde el amarillo claro al rojo oscuro la influencia global tomando como base la Figura 5-5.

Con esta representación se resaltan muy bien los parámetros que más afectan. Estos coinciden con lo esperado en la mayoría de los casos, como se comenta a continuación:

- Las uniones con las interfaces conductivas. Es el caso de los nodos 36, 37, 38 y 39, que representan los Hot Elements y Cold Element respectivamente, aunque 


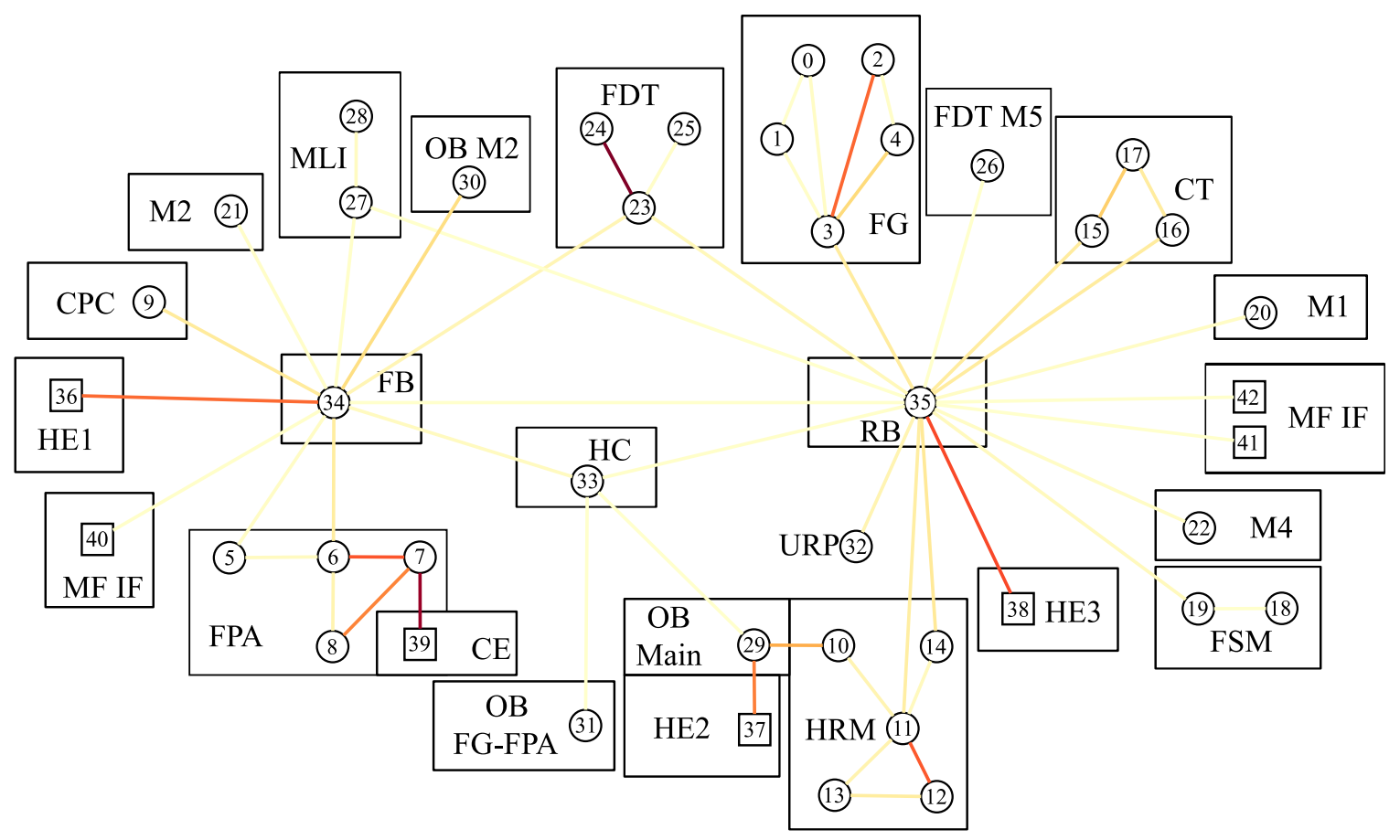

Figura 5-8: PHI O-Unit. Influencia global de los parámetros.

no ocurre lo mismo con las 3 patas, nodos 41, 42 y 43. En este caso, el diseño de la unidad es tal que el calor conducido a través de las patas es mínimo. De esta forma se cumple uno de los requisitos impuestos por el vehículo.

- Los parámetros que relacionan elementos que tienen disipación ya sea electrónica interna o carga solar. Este es el caso de los nodos 2, 12, 24 que representan el FG, y los LCVRs tanto del FDT como del HRT respectivamente.

De esta manera, de los 53 parámetros iniciales, nos quedamos sólo con 39, habiendo descartado $(0,1),(1,3),(2,4),(5,6),(5,34),(18,19),(23,25),(26,35),(27,35)$, $(29,33),(31,33),(34,35),(35,41)$ y $(35,42)$.

\subsubsection{Condiciones iniciales}

El valor inicial de los parámetros ha sido establecido durante el proceso de reducción y siguiendo los criterios del ajuste manual. Es decir, el proceso de actualización del modelo empieza con unos valores iniciales de los parámetros que deberían situar a las temperaturas del modelo cerca de las temperaturas de referencia. Estos $G_{L} \mathrm{~s}$ se han calculado en base a un estimación durante el proceso de reducción. Por ejem- 
plo, se intenta mantener el valor de conductancia lineal entre dos grupos de nodos si estos luego se reducen a un nodo cada uno. En otras ocasiones, se vuelve a estimar la conductacia en base a la distancia entre nodos, materiales y áreas. Aún así estas estimaciones a menudo resultan en valores de parámetros que están alejados de ser los que producen resultados satisfactorios.

En la Tabla 5.6 se muestra el valor inicial de los parámetros seleccionados. Dado que en este caso todos los parámetros seleccionados coinciden con $G_{L} \mathrm{~s}$, las unidades de estos son $[\mathrm{W} / \mathrm{K}]$. Con estos parámetros iniciales el modelo todavía está lejos de cumplir con los criterios de ajuste:

- En el caso DC2 (caliente operativo), hay una diferencia máxima de $21.3{ }^{\circ} \mathrm{C}$ y mínima de $0.3^{\circ} \mathrm{C}$. La diferencia media de todos los nodos es de $8.7^{\circ} \mathrm{C}$ con una desviación estándar de $4.86^{\circ} \mathrm{C}$.

El $86 \%$ de los nodos están fuera del margen admitido. Es decir, la diferencia de temperatura del $86 \%$ de los nodos del modelo respecto a su temperatura de referencia es mayor a $3{ }^{\circ} \mathrm{C}$.

- En el caso PC1 (frío operativo), la diferencia máxima es de $51.2{ }^{\circ} \mathrm{C}$ y la mínima de $0.3{ }^{\circ} \mathrm{C}$. La diferencia media es de $9.9^{\circ} \mathrm{C}$ con una desviación estándar de 15.81 ${ }^{\circ} \mathrm{C}$.

El $56 \%$ de los nodos tienen una diferencia de temperatura mayor a $3{ }^{\circ} \mathrm{C}$ respecto de la temperatura de referencia.

En cuanto al condicionamiento, el sistema está indeterminado ya que el número de nodos es 35 y el de parámetros es 39, si bien al tratarse de dos casos de carga el número total de datos es 70 . 
Tabla 5.6: PHI O-Unit. Valor inicial de los parámetros seleccionados.

\begin{tabular}{|l|c|}
\hline Parámetro & Valor Inicial $[\mathrm{W} / \mathrm{K}]$ \\
\hline$(0,3)$ & 0.100 \\
$(2,3)$ & 0.500 \\
$(3,4)$ & 0.020 \\
$(3,35)$ & 0.016 \\
$(6,7)$ & 0.055 \\
$(6,8)$ & 0.007 \\
$(6,34)$ & 0.018 \\
$(7,8)$ & 0.050 \\
$(7,39)$ & 0.450 \\
$(9,34)$ & 0.035 \\
$(10,11)$ & 0.150 \\
$(10,29)$ & 0.080 \\
$(11,12)$ & 0.016 \\
$(11,13)$ & 0.545 \\
$(11,14)$ & 0.150 \\
$(11,35)$ & 0.040 \\
$(12,13)$ & 0.180 \\
$(14,35)$ & 0.009 \\
$(15,17)$ & 0.001 \\
$(15,35)$ & 0.700 \\
$(16,17)$ & 0.570 \\
$(16,35)$ & 0.090 \\
$(19,35)$ & 0.150 \\
$(20,35)$ & 0.050 \\
$(21,34)$ & 0.500 \\
$(22,35)$ & 0.015 \\
$(23,24)$ & 0.150 \\
$(23,34)$ & 0.550 \\
$(23,35)$ & 0.700 \\
$(27,28)$ & 0.550 \\
$(27,34)$ & 0.550 \\
$(29,37)$ & 0.015 \\
$(30,34)$ & 0.030 \\
$(32,35)$ & 0.600 \\
$(33,34)$ & 0.500 \\
$(33,35)$ & 0.550 \\
$(34,36)$ & 0.650 \\
$(34,40)$ & 0.002 \\
$(35,38)$ & 0.100 \\
& \\
\hline
\end{tabular}


Tabla 5.7: PHI O-Unit. Temperaturas iniciales.

\begin{tabular}{|c|c|c|c|c|c|c|c|}
\hline \multirow[b]{2}{*}{ Nodo } & \multirow[b]{2}{*}{ Etiqueta } & \multicolumn{2}{|c|}{$\begin{array}{l}\text { Temperatura } \\
\text { Referencia }{ }^{\circ} \mathrm{C}\end{array}$} & \multicolumn{2}{|c|}{$\begin{array}{c}\text { Temperatura } \\
\text { Inicial }{ }^{\circ} \mathrm{C}\end{array}$} & \multicolumn{2}{|c|}{$\Delta T^{\circ} \mathrm{C}$} \\
\hline & & $\mathrm{DC} 2$ & $\mathrm{PC} 1$ & $\mathrm{DC} 2$ & PC1 & $\mathrm{DC} 2$ & PC1 \\
\hline 0 & FG Etalon & 65.9 & 66.5 & 66.21 & 17.04 & -0.3 & 49.5 \\
\hline 1 & FG Pre Filter & 65.2 & 63.3 & 66.14 & 16.77 & -0.9 & 46.5 \\
\hline 2 & FG Struct Ext & 57.0 & 14.2 & 65.72 & 14.68 & -8.7 & -0.5 \\
\hline 3 & FG Struct Int & 65.9 & 65.7 & 66.26 & 17.22 & -0.4 & 48.5 \\
\hline 4 & FG Lenses & 57.4 & 17.5 & 63.99 & 5.67 & -6.6 & 11.8 \\
\hline 5 & FPA Baffle & 51.5 & -18.3 & 59.91 & -16.75 & -8.4 & -1.5 \\
\hline 6 & FPA Sturcture & 46.8 & -16.9 & 45.92 & -16.30 & 0.9 & -0.6 \\
\hline 7 & FPA APS & 2.1 & -20.7 & 1.29 & -21.29 & 0.8 & 0.6 \\
\hline 8 & FPA PCB & 53.3 & -9.5 & 34.56 & -6.08 & 18.7 & -3.4 \\
\hline 9 & $\mathrm{CPC} \mathrm{CPC}$ & 53.6 & -16.4 & 64.79 & -9.10 & -11.2 & -7.3 \\
\hline 10 & HRM FS0 & 59.7 & -17.6 & 77.53 & -8.32 & -17.8 & -9.3 \\
\hline 11 & HRM Structure & 56.4 & -11.5 & 70.19 & -6.79 & -13.8 & -4.7 \\
\hline 12 & HRM LCVR & 75.0 & 45.2 & 71.74 & 0.45 & 3.3 & 44.7 \\
\hline 13 & HRM Polarizer & 78.5 & 46.5 & 71.08 & -4.75 & 7.4 & 51.2 \\
\hline 14 & HRM FS1 & 54.8 & -14.5 & 69.78 & -7.49 & -15.0 & -7.0 \\
\hline 15 & CT Structire & 54.1 & -16.3 & 62.34 & -14.40 & -8.2 & -1.9 \\
\hline 16 & CT Detector & 55.6 & -14.7 & 68.87 & -5.61 & -13.3 & -9.1 \\
\hline 17 & CT PCB & 64.2 & -4.6 & 69.92 & -4.26 & -5.7 & -0.3 \\
\hline 18 & FSM M3 & 52.4 & -17.2 & 63.11 & -13.37 & -10.7 & -3.8 \\
\hline 19 & FSM FS2 & 54.0 & -15.3 & 63.18 & -13.32 & -9.2 & -2.0 \\
\hline 20 & M1 M1 & 52.0 & -18.7 & 62.20 & -14.89 & -10.2 & -3.8 \\
\hline 21 & M2 M2 & 51.1 & -19.8 & 59.97 & -16.97 & -8.9 & -2.8 \\
\hline 22 & M4 M4 & 52.7 & -13.6 & 61.66 & -14.23 & -9.0 & 0.6 \\
\hline 23 & FDT Struc & 54.9 & -17.6 & 61.72 & -15.63 & -6.8 & -2.0 \\
\hline 24 & FDT LCVR & 74.8 & -5.4 & 67.02 & -13.71 & 7.8 & 8.3 \\
\hline 25 & FDT Lenses & 54.2 & -18.0 & 61.83 & -15.61 & -7.6 & -2.4 \\
\hline 26 & FDT M5 & 51.9 & -18.5 & 61.73 & -14.79 & -9.8 & -3.7 \\
\hline 27 & MLI Internal Layer & 52.9 & -18.5 & 61.16 & -16.40 & -8.3 & -2.1 \\
\hline 28 & MLI External Layer & 50.3 & -19.9 & 60.63 & -16.48 & -10.3 & -3.4 \\
\hline 29 & OB Main & 54.4 & -19.0 & 75.74 & -12.78 & -21.3 & -6.2 \\
\hline 30 & $\mathrm{OB} \mathrm{M} 2$ & 58.4 & -19.5 & 66.34 & -16.95 & -7.9 & -2.5 \\
\hline 31 & OB FPA-FG & 53.7 & -16.3 & 62.08 & -15.06 & -8.4 & -1.2 \\
\hline 32 & URP URP & 50.7 & -19.5 & 60.50 & -15.33 & -9.8 & -4.2 \\
\hline 33 & $\mathrm{HC} \mathrm{HC}$ & 52.5 & -18.8 & 61.15 & -16.32 & -8.6 & -2.5 \\
\hline 34 & FB FB & 51.6 & -19.7 & 59.80 & -16.98 & -8.2 & -2.7 \\
\hline 35 & RB RB & 51.6 & -18.7 & 61.73 & -14.78 & -10.1 & -3.9 \\
\hline
\end{tabular}




\subsubsection{Resultados}

El proceso de cálculo se empieza partiendo de los valores de los parámetros mostrados en la Tabla 5.6 y se empieza a iterar según se ha explicado en el apartado 3.2.3. Después de 13 iteraciones se ha logrado reducir el RSS de $164.14{ }^{\circ} \mathrm{C}$ hasta 2.27 ${ }^{\circ} \mathrm{C}$ (Figura 5-9). El tiempo de cálculo necesario ha sido de 6 segundos. En la Tabla 5.8 se muestra el valor encontrado para estos parámetros.

Con este conjunto de valores, las temperaturas que estima el modelo matemático están presentados en la Tabla 5.9, junto con la diferencia respecto a las temperaturas de referencia, donde puede observarse que:

- En el caso DC2 (caliente operativo), se ha pasado de una diferencia máxima de $21.3{ }^{\circ} \mathrm{C}$ y mínima de $0.3{ }^{\circ} \mathrm{C}$ a una máxima de $2.9{ }^{\circ} \mathrm{C}$ y mínima de $0.02{ }^{\circ} \mathrm{C}$. La diferencia media se ha reducido de $8.7^{\circ} \mathrm{C}$ a $1.7^{\circ} \mathrm{C}$ con una desviación estándar de $0.78^{\circ} \mathrm{C}$.

Todos los nodos están dentro del margen de temperatura admitido $\left(\left|T_{i}-T_{R_{i}}\right|<\right.$ $\left.3^{\circ} \mathrm{C}\right)$.

- En el caso PC1 (frío operativo), la diferencia máxima se ha reducido de $51.2{ }^{\circ} \mathrm{C}$ a $2.6{ }^{\circ} \mathrm{C}$ y la mínima de $0.3{ }^{\circ} \mathrm{C}$ a $0.11{ }^{\circ} \mathrm{C}$. La diferencia media se ha reducido de $9.9{ }^{\circ} \mathrm{C}$ a $1.0^{\circ} \mathrm{C}$ con una desviación estándar de $0.78{ }^{\circ} \mathrm{C}$.

Todos los nodos están dentro del margen admitido.

Queda por comprobar el criterio de los flujos calor a través de las interfaces. El algoritmo GIPI no está teniendo en cuenta el ajuste de los parámetros para que este flujo sea igual que en el modelo detallado. Aún así, si se consigue reproducir las temperaturas del modelo, la distribución de flujos se espera que sea muy similar. En las Tablas 5.10 y 5.11 están recopilados estos flujos obtenidos con los parámetros iniciales y con los parámetros finales para los dos casos de carga ajustados. Entre paréntesis se ha calculado la diferencia respecto al obtenido con el DTMM. En ambos casos el error se ha reducido pero aún así no siempre se encuentra dentro del rango $(<10 \%)$ en todas las interfaces.

Hay que tener en cuenta que en el caso PC1 (frío operativo) los valores absolutos 
de los flujos de calor son muy pequeños, del orden de los $4 \mathrm{~W}$ el más elevado, por lo que un variación de solo $1 \mathrm{~W}$ supone un error del $25 \%$. Si nos fijamos en términos absolutos la desviación máxima es menor a $1 \mathrm{~W}$ en todas las interfaces. 
Tabla 5.8: PHI O-Unit. Valor final de los parámetros seleccionados.

\begin{tabular}{|l|c|}
\hline Parámetro & Valor final $[\mathrm{W} / \mathrm{K}]$ \\
\hline$(0,3)$ & 0.034 \\
$(2,3)$ & 0.015 \\
$(3,4)$ & 0.009 \\
$(3,35)$ & 0.001 \\
$(6,7)$ & 0.031 \\
$(6,8)$ & 0.150 \\
$(6,34)$ & 0.080 \\
$(7,8)$ & 0.016 \\
$(7,39)$ & 0.240 \\
$(9,34)$ & 0.170 \\
$(10,11)$ & 0.051 \\
$(10,29)$ & 0.218 \\
$(11,12)$ & 0.009 \\
$(11,13)$ & 0.001 \\
$(11,14)$ & 0.080 \\
$(11,35)$ & 0.052 \\
$(12,13)$ & 0.090 \\
$(14,35)$ & 0.159 \\
$(15,17)$ & 0.055 \\
$(15,35)$ & 0.207 \\
$(16,17)$ & 0.010 \\
$(16,35)$ & 0.091 \\
$(19,35)$ & 0.602 \\
$(20,35)$ & 0.168 \\
$(21,34)$ & 0.206 \\
$(22,35)$ & 0.014 \\
$(23,24)$ & 0.023 \\
$(23,34)$ & 0.172 \\
$(23,35)$ & 0.162 \\
$(27,28)$ & 0.002 \\
$(27,34)$ & 0.855 \\
$(29,37)$ & 0.908 \\
$(30,34)$ & 0.083 \\
$(32,35)$ & 0.106 \\
$(33,34)$ & 0.054 \\
$(33,35)$ & 0.296 \\
$(34,36)$ & 2.066 \\
$(34,40)$ & 0.022 \\
$(35,38)$ & 1.043 \\
& \\
\hline
\end{tabular}


Tabla 5.9: PHI O-Unit. Temperaturas finales.

\begin{tabular}{|c|c|c|c|c|c|c|c|}
\hline \multirow[b]{2}{*}{ Nodo } & \multirow[b]{2}{*}{ Etiqueta } & \multicolumn{2}{|c|}{$\begin{array}{l}\text { Temperatura } \\
\text { Referencia }{ }^{\circ} \mathrm{C}\end{array}$} & \multicolumn{2}{|c|}{$\begin{array}{c}\text { Temperatura } \\
\text { Final }{ }^{\circ} \mathrm{C}\end{array}$} & \multicolumn{2}{|c|}{$\Delta T^{\circ} \mathrm{C}$} \\
\hline & & $\mathrm{DC} 2$ & PC1 & $\mathrm{DC} 2$ & PC1 & $\mathrm{DC} 2$ & $\mathrm{PC} 1$ \\
\hline 0 & FG Etalon & 65.9 & 66.5 & 68.03 & 66.26 & 2.13 & 0.24 \\
\hline 1 & FG Pre Filter & 65.2 & 63.3 & 67.81 & 65.29 & 2.61 & 1.99 \\
\hline 2 & FG Struct Ext & 57.0 & 14.2 & 58.01 & 12.84 & 1.01 & 1.36 \\
\hline 3 & FG Struct Int & 65.9 & 65.7 & 68.47 & 68.19 & 2.57 & 2.49 \\
\hline 4 & FG Lenses & 57.4 & 17.5 & 58.69 & 15.79 & 1.29 & 1.71 \\
\hline 5 & FPA Baffle & 51.5 & -18.3 & 53.57 & -18.70 & 2.07 & 0.40 \\
\hline 6 & FPA Structure & 46.8 & -16.9 & 49.19 & -14.97 & 2.39 & 1.93 \\
\hline 7 & FPA APS & 2.1 & -20.7 & 3.09 & -20.89 & 0.99 & 0.19 \\
\hline 8 & FPA PCB & 53.3 & -9.5 & 50.62 & -9.70 & 2.68 & 0.20 \\
\hline 9 & $\mathrm{CPC} \mathrm{CPC}$ & 53.6 & -16.4 & 56.16 & -15.55 & 2.56 & 0.85 \\
\hline 10 & HRM FS0 & 59.7 & -17.6 & 61.75 & -16.90 & 2.05 & 0.70 \\
\hline 11 & HRM & 56.4 & -11.5 & 58.62 & -11.79 & 2.22 & 0.29 \\
\hline 12 & HRM LCVR & 75.0 & 45.2 & 76.02 & 46.36 & 1.02 & 1.16 \\
\hline 13 & HRM Polarizer & 78.5 & 46.5 & 78.25 & 45.02 & 0.25 & 1.48 \\
\hline 14 & HRM FS1 & 54.8 & -14.5 & 56.73 & -15.21 & 1.93 & 0.71 \\
\hline 15 & CT Structure & 54.1 & -16.3 & 56.95 & -14.04 & 2.85 & 2.26 \\
\hline 16 & CT Detector & 55.6 & -14.7 & 55.75 & -15.57 & 0.15 & 0.87 \\
\hline 17 & CT PCB & 64.2 & -4.6 & 65.41 & -2.93 & 1.21 & 1.67 \\
\hline 18 & FSM M3 & 52.4 & -17.2 & 54.33 & -17.37 & 1.93 & 0.17 \\
\hline 19 & FSM FS2 & 54.0 & -15.3 & 54.32 & -17.36 & 0.32 & 2.06 \\
\hline 20 & M1 M1 & 52.0 & -18.7 & 54.33 & -17.74 & 2.33 & 0.96 \\
\hline 21 & M2 M2 & 51.1 & -19.8 & 53.55 & -19.03 & 2.45 & 0.77 \\
\hline 22 & $\mathrm{M} 4$ & 52.7 & -13.6 & 54.63 & -16.02 & 1.93 & 2.42 \\
\hline 23 & FDT Structure Tube & 54.9 & -17.6 & 55.62 & -17.81 & 0.72 & 0.21 \\
\hline 24 & FDT LCVR & 74.8 & -5.4 & 77.53 & -8.01 & 2.73 & 2.61 \\
\hline 25 & FDT Lenses & 54.2 & -18.0 & 55.82 & -17.78 & 1.62 & 0.22 \\
\hline 26 & FDT M5 & 51.9 & -18.5 & 53.85 & -17.79 & 1.95 & 0.71 \\
\hline 27 & MLI Internal Layer & 52.9 & -18.5 & 54.71 & -18.39 & 1.81 & 0.11 \\
\hline 28 & MLI External Layer & 50.3 & -19.9 & 50.32 & -19.79 & 0.02 & 0.11 \\
\hline 29 & OB Main & 54.4 & -19.0 & 55.96 & -19.13 & 1.56 & 0.13 \\
\hline 30 & OB M2 & 58.4 & -19.5 & 57.32 & -19.03 & 1.08 & 0.47 \\
\hline 31 & OB FPA-FG & 53.7 & -16.3 & 55.03 & -16.83 & 1.33 & 0.53 \\
\hline 32 & URP URP & 50.7 & -19.5 & 52.30 & -18.67 & 1.60 & 0.83 \\
\hline 33 & $\mathrm{HC} \mathrm{HC}$ & 52.5 & -18.8 & 54.71 & -18.39 & 2.21 & 0.41 \\
\hline 34 & $\mathrm{FB} \mathrm{FB}$ & 51.6 & -19.7 & 53.14 & -19.07 & 1.54 & 0.63 \\
\hline 35 & RB RB & 51.6 & -18.7 & 53.83 & -17.79 & 2.23 & 0.91 \\
\hline
\end{tabular}


Tabla 5.10: Flujos de calor a través de las interfaces en el caso DC2 (caliente operativo).

\begin{tabular}{|l|c|cc|cc|}
\hline Interfaz & Referencia & \multicolumn{2}{|c|}{ Parámetros Iniciales } & \multicolumn{2}{|c|}{ Parámetros encontrados } \\
\hline HE IF 1 & $6.8 \mathrm{~W}$ & $9.9 \mathrm{~W}$ & $(46 \%)$ & $7.0 \mathrm{~W}$ & $(3 \%)$ \\
HE IF 2 & $7.1 \mathrm{~W}$ & $3.6 \mathrm{~W}$ & $(-49 \%)$ & $6.7 \mathrm{~W}$ & $(-6 \%)$ \\
HE IF 3 & $4.7 \mathrm{~W}$ & $3.3 \mathrm{~W}$ & $(-30 \%)$ & $4.7 \mathrm{~W}$ & $(0 \%)$ \\
CE IF & $3.1 \mathrm{~W}$ & $5.1 \mathrm{~W}$ & $(65 \%)$ & $3.1 \mathrm{~W}$ & $(0 \%)$ \\
Feet & $0.1 \mathrm{~W}$ & $0.1 \mathrm{~W}$ & $(0.0 \mathrm{~W})$ & $0.5 \mathrm{~W}$ & $(0.4 \mathrm{~W})$ \\
S/C (Radiación) & $0.2 \mathrm{~W}$ & $<0.05 \mathrm{~W}$ & $(0.2 \mathrm{~W})$ & $<0.01 \mathrm{~W}$ & $(0.2 \mathrm{~W})$ \\
\hline
\end{tabular}

Tabla 5.11: Flujos de calor a través de las interfaces en el caso PC1 (frío operativo).

\begin{tabular}{|l|c|cl|ll|}
\hline Interfaz & Referencia & \multicolumn{2}{|c|}{ Parámetros Iniciales } & \multicolumn{2}{|l|}{ Parámetros encontrados } \\
\hline HE IF 1 & $1.4 \mathrm{~W}$ & $3.1 \mathrm{~W}$ & $(121 \%)$ & $2.1 \mathrm{~W}$ & $(50 \%)$ \\
HE IF 2 & $1.3 \mathrm{~W}$ & $1.1 \mathrm{~W}$ & $(-15 \%)$ & $1.2 \mathrm{~W}$ & $(-8 \%)$ \\
HE IF 3 & $3.8 \mathrm{~W}$ & $1.3 \mathrm{~W}$ & $(-66 \%)$ & $2.9 \mathrm{~W}$ & $(-24 \%)$ \\
CE IF & $0.9 \mathrm{~W}$ & $1.9 \mathrm{~W}$ & $(111 \%)$ & $1.0 \mathrm{~W}$ & $(11 \%)$ \\
Feet & $0.1 \mathrm{~W}$ & $0.1 \mathrm{~W}$ & $(<0,1 \mathrm{~W})$ & $0.3 \mathrm{~W}$ & $(0.2 \mathrm{~W})$ \\
S/C (Radiación) & $0.1 \mathrm{~W}$ & $<0,1 \mathrm{~W}$ & $(0.2 \mathrm{~W})$ & $0.1 \mathrm{~W}$ & $(<0,1 \mathrm{~W})$ \\
\hline
\end{tabular}

\subsubsection{Influencia del coeficiente de variación máxima}

En el apartado 3.2.3 hemos definido $c_{v}$ cómo el coficiente de variación máxima. Con ayuda de este coeficiente se limita el porcentaje de cambio que puede tener un parámetro en cada iteración, y se previene que el parámetro salga del rango para el cual se considera válida la linealización de los términos radiativos y se evitan oscilaciones debidas a una matriz $\mathbf{M}$ mal condicionada. Se ha considerado como punto de partida una variación máxima de $50 \%$ ya que según las pruebas realizadas se comporta razonablemente bien (no se observan oscilaciones) en la mayoría de los casos.

Para poder explicar la influencia de este coeficiente, en el caso de PHI O-Unit se ha realizado el ajuste utilizando diferentes valores. Se han efectuado un total de 20 iteraciones en todos los casos variando $c_{v}$ de 0.1 hasta 2.0 en incrementos de 0.1 (Figura 5-9). El RSS mínimo alcanzado en 20 iteraciones se muestra en la Tabla 5.12. Hay que tener en cuenta que una vez que se ha llegado al valor mínimo la solución oscilará alrededor de ese conjunto de parámetros. Es lo que sucede utilizando un coeficiente $c_{v}$ entre 0.1 y 1.0 . 
En la Figura 5-9 se muestra la evolución del RSS en función del número de iteraciones. De esta gráfica se sacan las siguientes conclusiones:

- Para valores de $c_{v}$ menores que 1.0 (en rojo, verde y azul), el método converge.

- La velocidad de convergencia aumenta con $c_{v}$ hasta 0.9. De hecho al limitar la variación posible de los parámetros a un $10 \%$ y un $20 \%$ no se alcanza a la convergencia en 20 iteraciones.

- Con $c_{v}$ iguales a 0.6 y 0.8 existe un mínimo local para 9 iteraciones. Esto genera un falso punto de parada del algoritmo, ya que cuando se observa un aumento en el RSS se deja de iterar en condiciones normales.

- Con una tasa de variación del $90 \%\left(c_{v}=0,9\right)$ se consigue la mayor velocidad de convergencia.

- Existe un compromiso entre un RSS más bajo y la velocidad de convergencia. En este caso el valor de RSS más bajo encontrado se obtiene para $c_{v}=0,4$ en 13 iteraciones. Un valor muy similar se consigue con $c_{v}=0,7$ y en solo 8 iteraciones. 


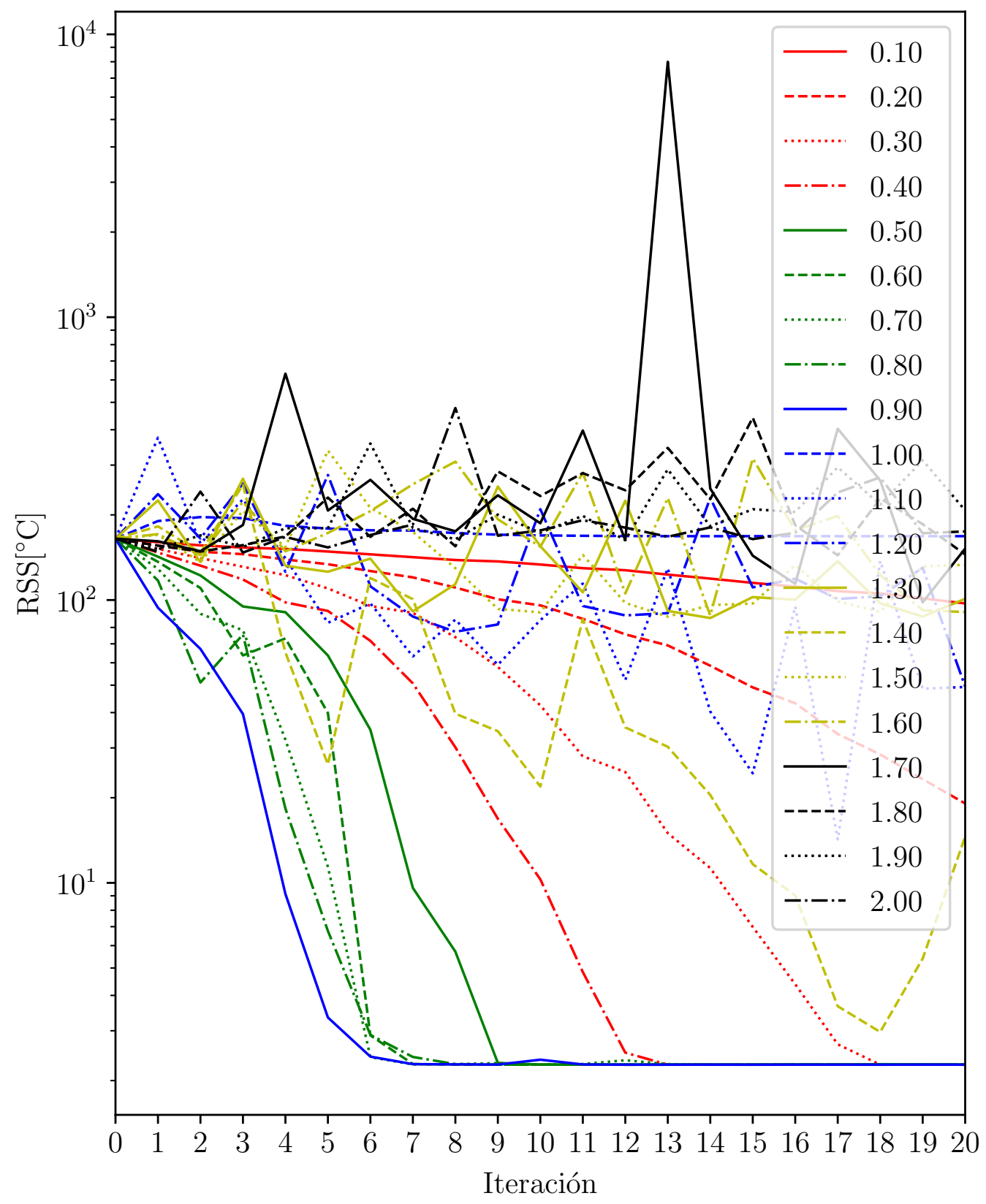

Figura 5-9: Variación del RSS con el número de iteraciones. Influencia del coeficiente de variación máxima. 
Tabla 5.12: RSS mínimo en 20 iteraciones

\begin{tabular}{|c|c|}
\hline$c_{v}$ & Mínimo RSS $\left[{ }^{\circ} \mathrm{C}\right]$ \\
\hline 0.10 & 97.259 \\
0.20 & 19.082 \\
0.30 & 2.276 \\
0.40 & 2.275 \\
0.50 & 2.276 \\
0.60 & 2.276 \\
0.70 & 2.276 \\
0.80 & 2.276 \\
0.90 & 2.275 \\
1.00 & 164.725 \\
1.10 & 14.23 \\
1.20 & 49.46 \\
1.30 & 86.36 \\
1.40 & 2.970 \\
1.50 & 87.372 \\
1.60 & 88.168 \\
1.70 & 98.116 \\
1.80 & 143.861 \\
1.90 & 151.534 \\
2.00 & 147.164 \\
\hline
\end{tabular}




\subsubsection{Comparación con otros métodos}

En este apartado se procede a comparar este método con otros métodos disponibles de optimización, como los que se encuentran en el paquete SciPy [53]. Para ello se ha creado una función objetivo que implementa la función a minimizar $E_{T}(\boldsymbol{X})$, definida en 2.2 .

Los primeros dos métodos utilizados para esta comparación son métodos que se llaman directos [67] ya que solo se hace uso del valor de la función objetivo. La gran ventaja de este tipo de métodos es que no requieren que la función sea diferenciable y además no requieren el cálculo de la primera o segunda derivada, que en muchas ocasiones puede llegar a ser muy costoso en términos computacionales.

El método símplex [68] más tarde mejorado por Nelder-Mead $[69,70]$ está indicado para la resolución de este tipo de problemas. En la implementación utilizada, Goa y Han, el método está optimizado para ser usado en problemas con un número grande de dimensiones y mejorado de manera que no quede atascado en un mínimo local [71]. Después de un total de 52035 iteraciones y 2333 segundos, el RSS obtenido es de $25.4{ }^{\circ} \mathrm{C}$.

El método de Powell [72,73] es un método considerado en principio ineficiente [67], ya que busca a lo largo de cada una de las direcciones (en el espacio de los parámetros, $\boldsymbol{X}$ ) y al final de cada iteración reemplaza una de estás direcciones por la que devuelve un valor más bajo de la función objetivo. En este caso, después de un total de 50 iteraciones y 594 segundos, el RSS obtenido es de 12.22. A pesar de ser a priori un método considerado más ineficiente que el del Nelder-Mead ha funcionado mejor con este problema.

En cuanto a los métodos indirectos, se ha utilizado uno de los más populares [74], el método BFGS, llamado así por el nombre de sus desarrolladores Broyden, Fletcher, Goldfarb y Shanno. Es un método quasi-Newtoniano con la particularidad de que utiliza una aproximación del la matriz hessiana en vez de tener que calcularla en cada iteración. Este método requiere que se especifique un parámetro de control $\epsilon$, que se corresponde con el delta utilizado para el cálculo del jacobiano. Este parámetro 
es el mismo para todos los parámetros e influye en la solución encontrada por el método. Utilizando un valor $\epsilon=0,001$ se ha encontrado la mejor solución después de 23 iteraciones y 172 segundos dando un RSS de $93.02{ }^{\circ} \mathrm{C}$.

Con el fin de poder utilizar los métodos quasi-Newtonianos para conjuntos muy grandes de parámetros se utilizan variaciones que permiten procesar las matrices hessianas (con tamaño $n \times \mathrm{x} n$, siendo $n$ el número de parámetros) utilizando unos cuantos vectores de tamaño $n$. Este es el caso del método Limited-Memory BFGS, que además en la implementación utilizada [75-77] permite poner límites a la variación de los parámetros. Con el método L-BFGS-B se obtuvo un RSS de $29.11^{\circ} \mathrm{C}$ en 103.7 segundos y 52 iteraciones.

Por último vamos a comparar con el algoritmo genético Differential Evolution $[78,79]$. Este algoritmo se considera también un método directo de minimización ya que no hace el cálculo de la derivadas de la función a minimizar. A diferencia de los otros métodos, este no requiere de un conjunto de valores iniciales sino que utiliza valores aleatorios en la primera generación que luego va mutando de acuerdo a la estrategia elegida. En este caso se ha utilizado la estrategia 'best1bin', que se recomienda para este tipo de problemas. A pesar de lo ineficiente de este tipo de métodos ya que no hacen uso del comportamiento de la función, generalmente arrojan buenos ajustes. La gran desventaja es el tiempo que toman ya que requieren un número de iteraciones muy alto. Después de cinco ejecuciones, se ha seleccionado la mejor (en cuanto a ajuste) que ha tardado más de 3 horas y media con 1660 iteraciones para llegar a un ajuste parecido al método propuesto, $\mathrm{RSS}=3.31{ }^{\circ} \mathrm{C}$.

En la Tabla 5.13 se agrupan los resultados de los métodos comparados. Para el cálculo del tiempo de ejecución de cada método se ha usado la media de 5 ejecuciones a excepción de para el método genético que se ha seleccionado el mejor de 5, ya que la solución varía con cada ejecución.

Por último vamos a comparar los parámetros encontrados con el método Differential Evolution. Solo tenemos en cuenta este método ya que es el único que ha llegado a un ajuste satisfactorio. En la Tabla 5.14 están comparados los valores finales de los parámetros obtenidos con estos dos métodos. Se ha añadido una columna que indica 
Tabla 5.13: Comparación de los diferentes métodos.

\begin{tabular}{|c|c|c|c|}
\hline Método & Mínimo RSS $\left[{ }^{\circ} \mathrm{C}\right]$ & Iteraciones & Tiempo $[\mathrm{s}]$ \\
\hline BFGS & 70.90 & 34 & 185 \\
L-BFGS-B & 29.11 & 52 & 103 \\
Nelder-Mead & 25.40 & 52035 & 2333 \\
Powell & 12.22 & 50 & 594 \\
Differential Evolution & 2.31 & 1660 & 13107 \\
\hline GIPI & 2.27 & 13 & 6 \\
\hline
\end{tabular}

la discrepancia, calculada como $X_{G I P I} / X_{D E}$. Es interesante fijarse que los valores que menor discrepancia tienen corresponden a los parámetros que más influencia global tienen, como por ejemplo los parámetros $(2,3),(7,34)$ y $(23,24)$ y los que muestran mayor discrepancia son aquellos que presentan menor influencia global, por ejemplo el $(16,17),(20,35)$ o el $(34,40)$.

Una comparación similar ha sido realizada por Anglada en [80], donde compara el algoritmo genético desarrollado por ellos con métodos basados en el cálculo del gradiente. Para ello utiliza un modelo de siete nodos y seis conductancias térmicas (radiativas y conductivas). Anglada llega a la conclusión de que el uso de de los algoritmos basados en el cálculo del gradiente no son adecuados por su imposibilidad de ajustar varios casos de carga en estado estacionario. De manera que si ajustas un caso, el resto de casos puede no estar ajustado. El algoritmo genético presentando por ellos resuelve este problema con tiempos de ejecución similares.

$\mathrm{El}$ algoritmo presentado en esta tesis resuelve el problema de ajustar varios casos de carga al mismo tiempo además demostrar unas velocidades de convergencia comparables o mejores al resto de métodos, como se ha visto aplicado a la O-Unit de PHI. 
Tabla 5.14: PHI O-Unit. Comparación de los parámetros finales entre el método propuesto y el algoritmo genético.

\begin{tabular}{|c|c|c|c|}
\hline Parámetro & GIPI $\left[\mathrm{W} \mathrm{K}^{-1}\right]$ & Differential Evolution [ $\left.\mathrm{W} \mathrm{K}^{-1}\right]$ & Discrepancia \\
\hline$(0,3)$ & 0.034 & 0.046 & 0.73 \\
\hline$(2,3)$ & 0.015 & 0.015 & 1.00 \\
\hline$(3,4)$ & 0.009 & 0.009 & 1.00 \\
\hline$(3,35)$ & 0.001 & 0.001 & 0.93 \\
\hline$(6,7)$ & 0.031 & 0.031 & 0.98 \\
\hline$(6,8)$ & 0.150 & 0.152 & 0.99 \\
\hline$(6,34)$ & 0.080 & 0.083 & 0.97 \\
\hline$(7,8)$ & 0.016 & 0.016 & 1.03 \\
\hline$(7,39)$ & 0.240 & 0.241 & 1.00 \\
\hline$(9,34)$ & 0.170 & 0.158 & 1.08 \\
\hline$(10,11)$ & 0.051 & 0.052 & 0.99 \\
\hline$(10,29)$ & 0.218 & 0.216 & 1.01 \\
\hline$(11,12)$ & 0.009 & 0.009 & 1.00 \\
\hline$(11,13)$ & 0.001 & 0.001 & 0.83 \\
\hline$(11,14)$ & 0.080 & 0.109 & 0.73 \\
\hline$(11,35)$ & 0.052 & 0.036 & 1.47 \\
\hline$(12,13)$ & 0.090 & 0.092 & 0.98 \\
\hline$(14,35)$ & 0.159 & 0.201 & 0.79 \\
\hline$(15,17)$ & 0.055 & 0.029 & 1.89 \\
\hline$(15,35)$ & 0.207 & 0.117 & 1.78 \\
\hline$(16,17)$ & 0.010 & 0.035 & 0.30 \\
\hline$(16,35)$ & 0.091 & 0.228 & 0.40 \\
\hline$(19,35)$ & 0.602 & 0.754 & 0.80 \\
\hline$(20,35)$ & 0.168 & 0.399 & 0.42 \\
\hline$(21,34)$ & 0.206 & 0.302 & 0.68 \\
\hline$(22,35)$ & 0.014 & 0.016 & 0.90 \\
\hline$(23,24)$ & 0.023 & 0.023 & 0.99 \\
\hline$(23,34)$ & 0.172 & 0.179 & 0.96 \\
\hline$(23,35)$ & 0.162 & 0.127 & 1.27 \\
\hline$(27,28)$ & 0.002 & 0.002 & 0.98 \\
\hline$(27,34)$ & 0.855 & 1.250 & 0.68 \\
\hline$(29,37)$ & 0.908 & 0.912 & 1.00 \\
\hline$(30,34)$ & 0.083 & 0.084 & 0.98 \\
\hline$(32,35)$ & 0.106 & 0.093 & 1.14 \\
\hline$(33,34)$ & 0.054 & 0.180 & 0.30 \\
\hline$(33,35)$ & 0.296 & 0.444 & 0.67 \\
\hline$(34,36)$ & 2.066 & 0.838 & 2.47 \\
\hline$(34,40)$ & 0.022 & 1.328 & 0.02 \\
\hline$(35,38)$ & 1.043 & 0.931 & 1.12 \\
\hline
\end{tabular}




\section{Chapter 6}

\section{Conclusions and future work}

\subsection{Conclusions}

In the present doctoral dissertation a method for the correlation of thermal mathematical models in the context of spacecraft thermal design has been presented. The method is based on the minimization of the difference between the results of the model and the reference data, the problem is written in terms of the Jacobian matrix formulation and is solved using the Moore-Penrose pseudo-inverse formulation, generalized to include several load cases. The reference data can be either experimental data obtained from test or results obtained with a more detailed model.

The details of this method have been explained by using two simple mathematical models as examples. The first one, with only two nodes (in section 3), allows us to solve the equations analytically and study the characteristic of the solution, focusing on the problems that can appear when the Jacobian matrix is singular. In such a case, the Jacobian matrix can be analyzed looking for the singularity origin. Sometimes the singularities can be avoided by reducing the size of model as follows: 1) disregarding free parameters when they have no influence (matrix columns with small values) or non-distinguishable influence (linearly dependent columns), solving in this way the parameter observability problem, 2) disregarding nodes whose temperatures are no influenced by the parameters selected, and 3) condensing nodes when their temperatures follow the same trends (linearly dependent rows) thus solving the 
temperature observability problem. The second model with 4 nodes (in section 4), allows us to apply the method easily and study the possibilities that can appear when correlating thermal mathematical models, when different load cases are considered. A visualization of the error has been added to help understanding the nature of the minimization iterative process and the trajectory followed by the parameters of the model. Although this 4-node TMM may appear to be a non-realistic model, in fact it could represent a real reduced thermal mathematical model of some unit, as is the case of the PHI E-Unit presented in [65]. In addition, this model was used by [36] and showed itself to be good starting point for comparison.

The correlation of the thermal mathematical model to a reference temperature data set is a task that any thermal engineer must confront sooner or later in the common development path of a space project. The results of the models have to be in agreement with the temperatures measured in the TVAC tests or the flight data. The RTMM must (or intend to) predict the same temperatures that the corresponding DTMM. In any case the correlation must be carried out and the source of the reference data is not important. This correlation is usually done manually and requires an enormous effort in terms of time and therefore, cost. The intention of the method proposed is to provide the thermal engineer with the tools required to incorporate the model correlation to the normal workflow of the thermal design. This goal have been successfully reached by developing a tool that reads the .TMD files (from ESATANTMS) and the reference temperatures, and gives back the parameters that best fit those temperatures.

The method has been applied to the PHI O-Unit RTMM. The intention was to demonstrate that the correlation method can be applied to a larger model, but still small enough to make it understandable by a person from outside the project. The model has 35 temperature points to be correlated with the results from two load cases. This model has been presented and its parameters analyzed. A total of 39 parameters were selected for the correlation and the results provided by the correlated model shows that the temperatures obtained are inside the common requirements used in space programs. The difference in the calculated temperature with the initial 
values of the parameters, $X_{0}$ and the final values $X_{\text {final }}$ with respect to the reference temperature, $\boldsymbol{T}_{\boldsymbol{R}}$ is presented in the Figure 6-1. The temperature calculated with the initial values of the parameters, in red, has a discrepancy with the reference temperature up to $50{ }^{\circ} \mathrm{C}$. This discrepancy is reduced down to less than $3^{\circ} \mathrm{C}$ with the values found with the proposed method.

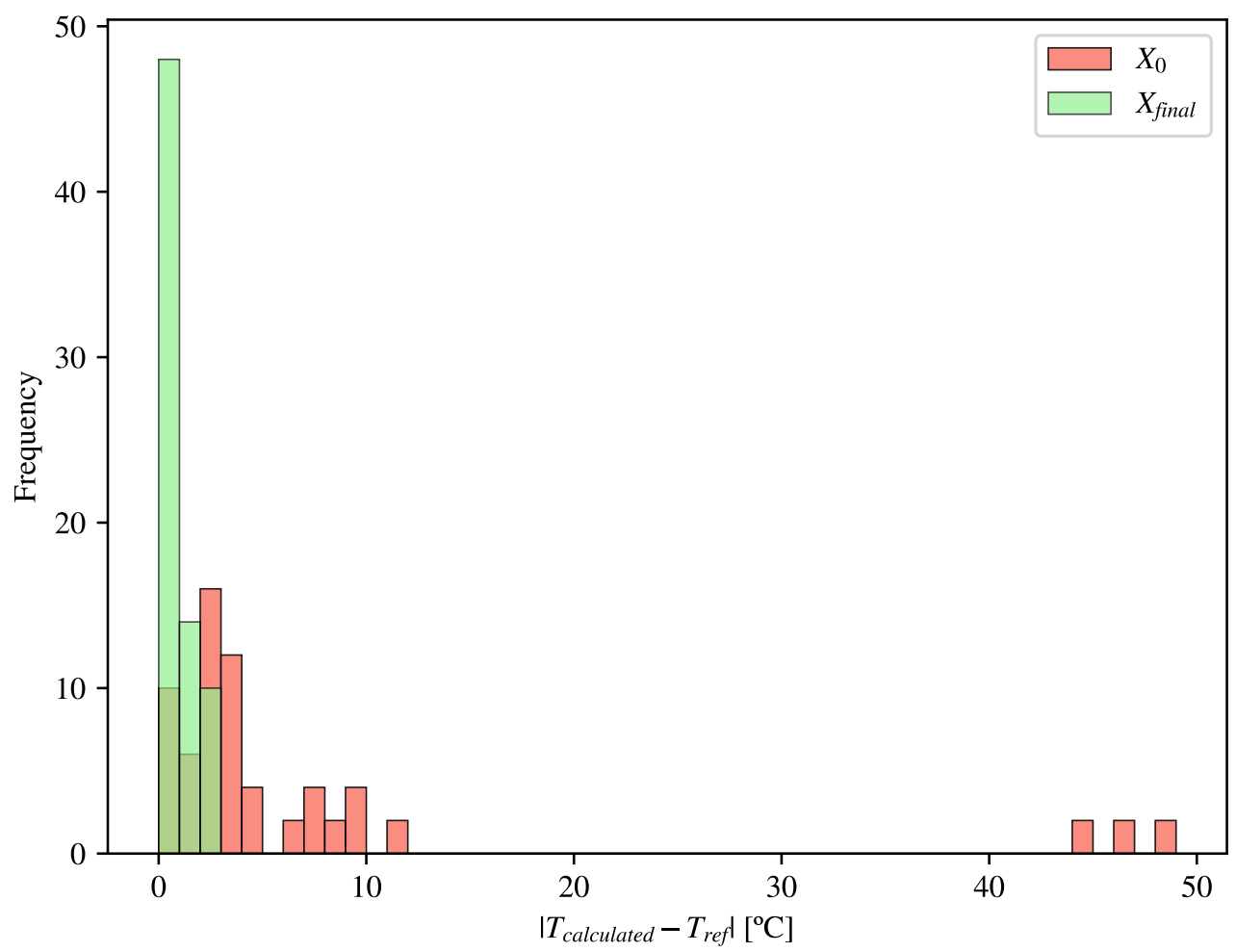

Figure 6-1: SO/PHI O-Unit. Distribution

Finally, the method has been compared with other minimization methods and the results show that the proposed correlation method proved to be the fastest and provided the best results.

The work presented in this doctoral dissertation can help advance in the process towards the automatization of the model-to-reference correlation methods. But, it is worth mentioning that a correlated model may not be a true representation of the reality and it should be used carefully if the parameters are outside the correlated points. The engineer experience will always be needed as it gives the feeling needed 
to model the reality.

\subsection{Future Work}

The work presented in this doctoral dissertation may serve as starting point for future studies. In this contezt, some improvements and ideas to work on have been found. All of them with the aim to ease the correlation process for the thermal engineer when updating a thermal mathematical model:

1. Study of the coefficient of maximum variation, $c_{v}$. During the test done in the present work, it has been found that during the process of updating the value of the parameters, every parameter has it own optimal $c_{v}$. Here, only a single value is used as is discussed in Section 5.3.4.

The result suggests that a particular $c_{v}$ for each parameter can accelerate the convergence of the solution meanwhile avoiding oscillations. The value of the $c_{v}$ may also be variable and dependent of the $\operatorname{RSS}^{1}$ of the model.

2. Definition of the correlation weights. The proposed method correlates the TMM by minimizing the difference of the temperatures calculated and the reference temperatures given, as defined in 2.2. In this expression the temperature of the nodes taken into account for the correlation process have the same weight. This situation is not always desirable, as in some cases the requirement for correlation is not the same in all the components/parts of the model. For instance an MLI is considered correlated if its temperature differs less than $20^{\circ} \mathrm{C}$ [4].

3. Correlation of transient analysis. It is a common practice to use the temperatures of the thermal balance test to correlate the TMM. These tests seek to find the stationary behavior of the system/unit to correlate the TMM against the temperatures at this state. The correlation method presented only uses one o more steady-state load cases to update the values of the parameters. Therefore, the thermal capacities of the model cannot be correlated and remain untouched as they are not present in the steady-state heat balance equation. The correla-

\footnotetext{
${ }^{1}$ Square Root of the Sum of Squares.
} 
tion of transient results of reference models will bring this information.

4. Including the heat fluxes to the correlation. Usually the RTMM is intended to be used to be integrated it in a higher hierarchical model. This is the case, for instance when integrating a payload model in a S/C platform model. The RTMM must behave as similar as possible as its corresponding DTMM, in terms of calculated temperatures and heat fluxes. The heat fluxes are key when for example, sizing a radiator.

The implementation of the method only considers the temperatures of the model, but it would be desirable to include the heat fluxes in the comparison.

5. Improving the use of constraints in the values of the parameters. The tool developed takes into account the value of the parameters by giving a minimum and maximum values. These constraints are checked before updating the parameters by limiting them (See Figure 3-3). The algorithm may be optimized by including the constraints in the Jacobian matrix formulation, as suggested from the theory of constrained optimization in [74].

6. Generalization of the implementation to be able to read the values of the physical parameters directly from the ESATAN-TMS files. Al the moment the physical properties are lost in the evaluation of the model linear conductors, $G_{L} \mathrm{~s}$. The $G_{L}$ S are the magnitudes read from ESATAN-TMS files and treated as parameters for the correlation. Therefore, they are losing therir original physical interpretation. 


\section{Bibliography}

[1] D. G. Gilmore, Spacecraft Thermal Control Handbook. Volume I. El Segundo, California, Aerospace Press, 2002, vol. I.

[2] J. Meseguer, I. Pérez-Grande, and A. Sanz-Andrés, Spacecraft Thermal Control. Woodhead Publishing, 2012.

[3] E. R. Division and Standards, "ECSS-M-ST-10C Project planning and implementation," p. 27, 2009.

[4] "Thermal analysis handbook, ECSS-E-HB-31-03A," pp. 36-42, 2016, division, ESA Requirements and Standards.

[5] R. Karam, Satellite Thermal Control for Systems Engineers. American Institute of Aeronautics and Astronautics, Inc, 2012.

[6] L. Jacques, "Thermal Design of OUFTI-1 Nanosatellite," Ph.D. dissertation, University of Liège, 2009.

[7] ITP Engines UK Ltd, "ESATAN-TMS Thermal User Manual," ITP Engines UK Ltd. Whetstone, Leicester, UK, Tech. Rep., 2014.

[8] S. Appel, R. Patrício, H. P. de Koning, and O. Pin, "Automatic Linear Conductor Generation Solution for Lumped Parameter Models," in 34th International Conference on Environmental Systems, 2004.

[9] J. H. Strutt, N. J. Stock, and C. J. Kirtley, "Lumped Parameter Thermal Conductor Generation for 3D Geometry," 44th International Conference on En- 
vironmental Systems, no. July, pp. 1-8, 2014. [Online]. Available: https://ttu-ir. tdl.org/ttu-ir/bitstream/handle/2346/59630/ICES-2014-297.pdf?sequence=1

[10] I. Garmendia, E. Anglada, H. Vallejo, and M. Seco, "Accurate calculation of conductive conductances in complex geometries for spacecrafts thermal models," Advances in Space Research, vol. 57, no. 4, pp. 1087-1097, 2016. [Online]. Available: http://dx.doi.org/10.1016/j.asr.2015.12.027

[11] A. M. Gómez-San-Juan, "Análisis de incertidumbres en sistemas de control térmico en ambientes espaciales," Ph.D. dissertation, Universidad Politécnica de Madrid, 2018.

[12] P. Planas Almazan, "Accuracy of Monte Carlo ray-tracing thermal radiation calculations: A practical discussion," in Sixth European Symposium on Space Environmental Control Systems, 1997, pp. 579-591.

[13] A. Avila, "JPL Thermal Design Modeling Philosophy and NASA-STD-7009 Standard for Models and Simulations - A Case Study," National Aeronautics and Space Administration, pp. 1-13, 2011.

[14] G. Fernández-Rico, "Quasi-Autonomous Spacecraft Thermal Model Reduction," Ph.D. dissertation, Universidad Politécnica de Madrid Escuela, Escuela Técnica Superior de Ingeniería Aeronáutica y del Espacio Doctoral, 2018.

[15] I. Torralbo, J. Piqueras et al., "Solar Orbiter PHI Electronics Unit Thermal Design," in European Space Thermal Engineering Workshop, 2018, pp. 579-591.

[16] F. Jouffroy, D. Charvet, M. Jacquiau, and A. Capitaine, "Automared Thermal Model Reduction for Telecom S/C Walls," in 18th European Thermal EECLS Software Workshop, 2004.

[17] M. Molina and C. Clemente, "Thermal Model Automatic Reduction: Algorithm and Validation Techniques," in 19th European Workshop on Thermal and ECLS Software, no. 36, 2005. 
[18] M. Molina and C. Clemente, "Thermal model automatic reduction: Algorithm and validation techniques," SAE Transactions, vol. 115, pp. 294-303, 2006.

[19] M. Bernard, T. Basset, F. Brunetti, and J. Etchells, "Thermal Model Reduction Tool," in 24th European Workshop on Thermal and ECLS Software, no. November, 2010, pp. 1-21.

[20] M. Bernard, J. Etchells, T. Basset, and F. Brunetti, "Thermal Model Reduction - Theory \& Application," in 40th International Conference on Environmental Systems (ICES), 2014, pp. 2-21.

[21] G. Fernández-Rico, I. Pérez-Grande, A. Sanz-Andres, I. Torralbo, and J. Woch, "Quasi-autonomous thermal model reduction for steady-state problems in space systems," Applied Thermal Engineering, vol. 105, pp. 456-466, 2016.

[22] Z. Jin, S. C. Joshi, G. J. J. Nesamani, P. K. Chan, T. M. Ying, and C. H. Goh, "Data Analysis and Correlation for Thermal Balance Test on a Micro-Satellite Model," Heat Transfer Engineering, vol. 31, no. 3, pp. 222-233, 2010.

[23] E. W. Grob, "Thermal Model Correlation of a Geosynchronous Weather Satellite," in 46th International Conference on Environmental Systems, no. July, 2016, pp. $1-13$.

[24] J. Klement, E. Anglada, and I. Garmendia, "Advances in automatic thermal model to test correlation in space industry," 46th International Conference on Environmental Systems, no. July, pp. 1-11, 2016.

[25] B. Frey, M. Trinoga, M. Hoppe, and W. Ebeling, "Development of an Automated Thermal Model Correlation Method and Tool," in 44th International Conference on Environmental Systems (ICES), no. 44, 2015.

[26] E. Anglada and I. Garmendia, "Correlation of thermal mathematical models for thermal control of space vehicles by means of genetic algorithms," Acta Astronautica journal, vol. 108, pp. 1-17, 2015. 
[27] S. de Palo, T. Malosti, and G. Filiddani, "Thermal Correlation of BepiColombo MOSIF 10 Solar Constants Simulation Test," 25th European Workshop on Thermal and ECLS Software, no. November, pp. 271-284, 2011.

[28] "Thermal model correlation using genetic algorithms," [Online; accessed July 2019]. [Online]. Available: \{https://exchange.esa.int/restricted/ model-correlation/\}

[29] F. Jouffroy, D. Tochon, and A. Capitaine, "Bibliografical study of optimisation methods with focus on Genetic Algorithm tecniques w.r.t. post-test thermal model correlation problem," Astrium, Tech. Rep., 2006.

[30] F. Jouffroy, "Test plan \& test models for verification of post-test thermal model correlation by using a stochastic global optimization method," Astrium, Tech. Rep., 2006.

[31] F. Jouffroy and N. Durand, "Thermal model correlation using Genetic Algorithms," in 21st European Workshop on Thermal and ECLS Software, no. October, 2007, pp. 1-14.

[32] F. Jouffroy, D. Tochon, and A. Capitaine, "Verification of feasibility of post-test thermal model correlation by using Genetic Algorithm: analysis of test plan results," EADS Astrium, Tech. Rep., 2007. [Online]. Available: https://exchange.esa.int/restricted/model-correlation/

[33] G. N. Vanderplaats, Numerical optimization techniques for engineering design: with applications. McGraw-Hill New York, 1984, vol. 1.

[34] R. Horst and P. M. Pardalos, Handbook of global optimization. Springer Science \& Business Media, 2013, vol. 2.

[35] R. L. Galski, F. L. De Sousa, F. M. Ramos, and I. Muraoka, "Spacecraft thermal design with the Generalized Extremal Optimization Algorithm," Inverse Problems in Science and Engineering, vol. 15, no. 1, pp. 61-75, 2004. 
[36] J. Klement, "On using Quasi-Newton algorithms of the Broyden class for modelto-test correlation," Journal of Aerospace Technology and Management, vol. 6, no. 4, pp. 407-414, 2014.

[37] C. G. Broyden, "A Class of Methods for Solving Nonlinear Simultaneous Equations," Mathematics of Computation, vol. 19, no. 92, p. 577, 1965.

[38] I. Garmendia and E. Anglada, "Thermal mathematical model correlation through genetic algorithms of an experiment conducted on board the International Space Station," Acta Astronautica, vol. 122, pp. 63-75, 2016.

[39] Z.-H. Zhan, J. Zhang, Y. Li, and H. S.-H. Chung, "Adaptive particle swarm optimization." IEEE transactions on systems, man, and cybernetics. Part B, Cybernetics : a publication of the IEEE Systems, Man, and Cybernetics Society, vol. 39, no. 6, pp. 1362-1381, 2009.

[40] T. Beck, A. Bieler, and N. Thomas, "Numerical thermal mathematical model correlation to thermal balance test using adaptive particle swarm optimization (APSO)," Applied Thermal Engineering, vol. 38, pp. 168-174, 2012.

[41] N. van Zijl, B. Zandbergen, and B. Benthem, "Correlating thermal balance test results with a thermal mathematical model using evolutionary algorithms," 27th European Space Thermal Analysis Workshop, no. December, pp. 89-108, 2013.

[42] J. Gross and S. Rudolph, "Geometry and simulation modeling in design languages," Aerospace Science and Technology, vol. 54, pp. 183-191, 2016. [Online]. Available: http://dx.doi.org/10.1016/j.ast.2016.03.003

[43] L. Collatz and W. Wetterling, Optimization Problems. Springer, New York, NY, 1975, vol. 17, no. 399. [Online]. Available: https://www.jstor.org/stable/ 3615195 ? origin $=$ crossref

[44] M. J. Colaço and G. S. Dulikravich, "Inverse and Optimization Problems in Heat Transfer," Journal of the Brazilian Society of Mechanical Sciences and Engineering, vol. XXVIII, no. 1, pp. 1-24, 2006. 
[45] J. V. Beck and K. J. Arnold, Parameter Estimation in Engineering and Science. John Wiley \& Sons, 1977.

[46] D. W. Marquard, "An Algorithm For Least-Squares Estimation Of Nonlinear Parameters," Journal of the Society for Industrial and Applied Mathematics, vol. 11, no. 2, pp. 431-441, 1963.

[47] J.-R. Tsai, "Overview of Satellite Thermal Analytical Model," Journal of Spacecraft and Rockets, vol. 41, no. 1, pp. 120-125, 2008.

[48] I. Pérez-Grande, A. Sanz-Andrés, C. Guerra, and G. Alonso, "Analytical study of the thermal behaviour and stability of a small satellite," Applied Thermal Engineering, vol. 29, no. 11-12, pp. 2567-2573, aug 2009. [Online]. Available: http://linkinghub.elsevier.com/retrieve/pii/S1359431108005036

[49] R. MacAusland, "The Moore-Penrose inverse and least squares," Math 420: Advanced Topics in Linear Algebra, 2014.

[50] A. Pyzara, B. Bylina, and J. Bylina, "The influence of a matrix condition number on iterative methods' convergence," in 2011 Federated Conference on Computer Science and Information Systems (FedCSIS), 2011, pp. 459-464.

[51] R. Aster, B. Borchers, and C. Thurber, Parameter estimation and inverse problems. Elsevier, 2013, vol. 40, no. 6.

[52] G. van Rossum, "Python tutorial," Centrum voor Wiskunde en Informatica (CWI), Amsterdam, Tech. Rep. CS-R9526, May 1995.

[53] E. Jones, T. Oliphant, P. Peterson et al., "SciPy: Open source scientific tools for Python," 2001-, [Online; accessed July 2019]. [Online]. Available: http://www.scipy.org/

[54] S. van der Walt and M. Aivazis, "The NumPy Array: A Structure for Efficient Numerical Computation, Computing in Science \& Engineering," Computing in 
Science and Engineering, vol. 13, no. 2, pp. 22-30, 2011. [Online]. Available: http://aip.scitation.org/doi/abs/10.1109/MCSE.2011.37

[55] J. D. Hunter, "Matplotlib: A 2D graphics environment," Computing in Science and Engineering, vol. 9, no. 3, pp. 99-104, 2007.

[56] T. H. Group, "Hdf5," 1987-, [Online; accessed July 2019]. [Online]. Available: https://support.hdfgroup.org/HDF5/whatishdf5.html

[57] R. C. Aster, B. Borchers, and C. H. Thunber, Chapter Three: Rank deficiency and ill-conditioning. Elsevier, 2019, no. 3.

[58] ITP Engines UK Ltd, "ESATAN-TMS thermal engineering manual," ITP Engines UK Ltd. Whetstone, Leicester, UK, Tech. Rep., 2015.

[59] S. K. Solanki, J. C. d. T. Iniesta, and W. et al., "The Polarimetric and Helioseismic Imager on Solar Orbiter," Astronomy \& Astrophysics, 2019. [Online]. Available: http://arxiv.org/abs/1903.11061

[60] I. Pérez Grande, I. Torralbo, G. Alonso, A. Gómez-Sanjuan, and G. FernándezRico, "Thermal Design of the ESA Solar Orbiter Instrument PHI," 46th International Conference on Environmental Systems (ICES), no. July, pp. 1-11, 2016. [Online]. Available: https://ttu-ir.tdl.org/ttu-ir/handle/2346/67685

[61] European Space Agency, "Solar orbiter," [Online; accessed July 2019]. [Online]. Available: http://sci.esa.int/solar-orbiter/

[62] Max Planck Institute, "Max planck institute for solar system research," [Online; accessed July 2019]. [Online]. Available: https://www.mps.mpg.de/en/

[63] Universidad Politécnica de Madrid, "Instituto universitario de microgravedad 'ignacio da riva'," [Online; accessed July 2019]. [Online]. Available: https://www.idr.upm.es

[64] I. Torralbo, I. Perez-Grande, A. Sanz-Andres, and J. Piqueras, "Correlation of spacecraft thermal mathematical models to reference data," Acta Astronautica, 
vol. 144, no. November 2017, pp. 305-319, 2018. [Online]. Available: https://doi.org/10.1016/j.actaastro.2017.12.033

[65] I. Torralbo, I. Perez-Grande, J. L. Gasent-Blesa, J. Piqueras, E. Sanchis-Kilders, P. Rodriguez, and A. Lopez, "Thermal Analysis of the Solar Orbiter PHI Electronics Unit," IEEE Transactions on Aerospace and Electronic Systems, vol. Early Access, 2019.

[66] ESA Requirements and Standards Division, "ECSS-E-ST-31C - Space Engineering, Thermal control general requirements," p. 65, 2008. [Online]. Available: http://ecss.nl/standard/ecss-e-st-31c-thermal-control/

[67] R. M. Lewis, V. Torczon, M. W. Trosset, and C. William, "Direct Search Methods : Then and Now Operated by Universities Space Research Association," Journal of Computational and Applied Mathematics, vol. 124, no. 1-2, pp. 191-207, 2000.

[68] W. Spendley, G. R. Hext, and F. R. Himsworth, "Sequential application of simplex designs in optimisation and evolutionary operation," Technometrics, vol. 4, no. 4, pp. 441-461, 1962.

[69] J. A. Nelder and R. Mead, "A Simplex Method for Function Minimization," The Computer Journal, vol. 7, no. 4, pp. 308-313, 1965. [Online]. Available: https://academic.oup.com/comjnl/article-lookup/doi/10.1093/comjnl/7.4.308

[70] F. Gao and L. Han, "Implementing the Nelder-Mead simplex algorithm with adaptive parameters," Computational Optimization and Applications, vol. 51, no. 1, pp. 259-277, 2012.

[71] J. C. Lagarias, J. C. Lagarias, J. A. Reeds, M. H. Wright, and P. E. Wright, "Convergence Properties of the Nelder-Mead Simplex Method in Low Dimensions," SIAM Journal of Optimization, vol. 9, no. 1, pp. 112 - 147, 1998. [Online]. Available: http://citeseer.ist.psu.edu/viewdoc/summary?doi=10.1.1. 120.6062 
[72] M. Powell, "An efficient method for finding the minimum of a function of several variables without calculating derivatives," The Computer Journal, vol. 7, no. 2, pp. $155-162,1964$.

[73] M. Wright, Direct search methods: Once scorned, now respectable. AddisonWesley, 1996, pp. 191-208.

[74] J. Nocedal and Wright Stephen J., Numerical Optimization. Springer, 1999. [Online]. Available: www.bioinfo.org.cn/\{ $\}$ wangchao/maa/Numerical $\{\backslash$ \}Optimization.pdf

[75] R. Byrd, P. Lu, J. Nocedal, and C. Zhu, "A Limited Memory Algorithm For Bound Constrained Optimization," SIAM Journal of Optimization, vol. 16, no. 5, pp. 1190-1208, 1995.

[76] C. Zhu, R. H. Byrd, P. Lu, and J. Nocedal, "Algorithm 778: L-BFGS-B: Fortran subroutines for large-scale bound-constrained optimization," ACM Transactions on Mathematical Software, vol. 23, no. 4, pp. 550-560, 1997.

[77] J. L. Morales and J. Nocedal, "Remark on Algorithm 778: L-BFGS-B: Fortran subroutines for large-scale bound-constrained optimization," ACM Transactions on Mathematical Software, vol. 38, no. 1, pp. 550-560, 2011.

[78] W. D. C. Franz Josef Ahlers et al., "Differential Evolution: for continuous function optimization," 2000-, [Online; accessed July 2019]. [Online]. Available: http://www1.icsi.berkeley.edu/ storn/code.html

[79] R. Storn and K. Price, "Differential Evolution - A Simple and EfficientHeuristic for Global Optimization over Continuous Spaces," Journal ofGlobal Optimization, vol. 11, no. 1, pp. 341-359, 1997.

[80] E. Anglada, L. Martinez-Jimenez, and I. Garmendia, "Performance of GradientBased Solutions versus Genetic Algorithms in the Correlation of Thermal Mathematical Models of Spacecrafts," International Journal of Aerospace Engineering, vol. 2017, pp. 1-12, 2017. 